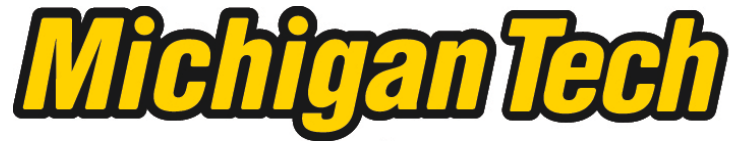 \\ Michigan Technological University Create the Future Digital Commons @ Michigan Tech
}

Dissertations, Master's Theses and Master's Reports - Open

Dissertations, Master's Theses and Master's

Reports

2013

\section{A COMPARATIVE STUDY OF HEURISTIC OPTIMIZATION ALGORITHMS}

Rohit A. Bhatia

Michigan Technological University

Follow this and additional works at: https://digitalcommons.mtu.edu/etds

Part of the Electrical and Computer Engineering Commons

Copyright 2013 Rohit A. Bhatia

\section{Recommended Citation}

Bhatia, Rohit A., "A COMPARATIVE STUDY OF HEURISTIC OPTIMIZATION ALGORITHMS", Master's report, Michigan Technological University, 2013.

https://doi.org/10.37099/mtu.dc.etds/702

Follow this and additional works at: https://digitalcommons.mtu.edu/etds

Part of the Electrical and Computer Engineering Commons 


\title{
A COMPARATIVE STUDY OF HEURISTIC OPTIMIZATION ALGORITHMS
}

By

Rohit A. Bhatia

\author{
A REPORT \\ Submitted in partial fulfillment of the requirements for the degree of \\ MASTER OF SCIENCE \\ In Electrical Engineering \\ MICHIGAN TECHNOLOGICAL UNIVERSITY \\ 2013
}

(C) 2013 Rohit A. Bhatia 
This report has been approved in partial fulfillment of the requirements for the Degree of MASTER OF SCIENCE in Electrical Engineering.

Department of Electrical and Computer Engineering

Report Advisor: Dr. Ashok Goel

Committee Member: Dr. Zhuo Feng

Committee Member: Dr. Laura E. Brown

Department Chair: Dr. Daniel R. Fuhrmann 


\section{Table of Contents}

Acknowledgements $\quad 4$

Abstract 5

1. Heuristic algorithms - Introduction 6

2. Non-adaptive simulated annealing

2.1 Overview 9

2.2 Working of the non-adaptive simulated annealing algorithm 11

$\begin{array}{ll}2.3 \text { Pseudocode } & 13\end{array}$

$\begin{array}{ll}2.4 \text { Parameterization } & 16\end{array}$

$\begin{array}{lr}2.5 \text { Algorithm output } & 18\end{array}$

3. Adaptive simulated annealing

$\begin{array}{ll}3.1 \text { Motivation } & 20\end{array}$

3.2 Working of the adaptive simulated annealing algorithm 21

3.3 Pseudocode 23

$\begin{array}{ll}3.4 \text { Algorithm output } & 25\end{array}$

4. Random restart hill climbing

$\begin{array}{ll}4.1 \text { Overview } & 27\end{array}$

4.2 Working of the random restart hill climbing algorithm 28

4.3 Pseudocode $\quad 30$

4.4 Algorithm output 33

5. Performance comparison

5.1 Performance comparison with a cost function of two variables 35

5.2 Performance comparison with a cost function of three variables 39

5.3 Performance comparison with a cost function of four variables 43

$\begin{array}{ll}\text { 6. Conclusions } & 47\end{array}$

$\begin{array}{ll}\text { Appendix } 1 & 48\end{array}$

$\begin{array}{ll}\text { References } & 74\end{array}$ 


\section{Acknowledgements}

I am grateful to my advisor Dr. Ashok Goel for his continuous support and guidance that fostered my learning in the subject area and helped me develop a strong understanding of the matter. Without Dr. Goel's support, this would have been an enormously difficult task to implement.

Additionally, I am grateful to Dr. Zhuo Feng and Dr. Laura E. Brown for being part of my defense committee and providing me their valuable feedback so as to get the most from my efforts.

I am also glad to have had access to resources at Michigan Technological University that made my learning experience very joyous and fulfilling.

Finally, I am extremely grateful to my family and friends, and my parents in particular for their unconditional support especially in times when I needed it. 


\section{Abstract}

Heuristic optimization algorithms are of great importance for reaching solutions to various real world problems. These algorithms have a wide range of applications such as cost reduction, artificial intelligence, and medicine. By the term cost, one could imply that that cost is associated with, for instance, the value of a function of several independent variables. Often, when dealing with engineering problems, we want to minimize the value of a function in order to achieve an optimum, or to maximize another parameter which increases with a decrease in the cost (the value of this function). The heuristic cost reduction algorithms work by finding the optimum values of the independent variables for which the value of the function (the "cost") is the minimum.

There is an abundance of heuristic cost reduction algorithms to choose from. We will start with a discussion of various optimization algorithms such as Memetic algorithms, force-directed placement, and evolution-based algorithms. Following this initial discussion, we will take up the working of three algorithms and implement the same in MATLAB.

The focus of this report is to provide detailed information on the working of three different heuristic optimization algorithms, and conclude with a comparative study on the performance of these algorithms when implemented in MATLAB. In this report, the three algorithms we will take in to consideration will be the non-adaptive simulated annealing algorithm, the adaptive simulated annealing algorithm, and random restart hill climbing algorithm. The algorithms are heuristic in nature, that is, the solution these achieve may not be the best of all the solutions but provide a means to reach a quick solution that may be a reasonably good solution without taking an indefinite time to implement. 


\section{Heuristic algorithms - Introduction}

There are several algorithms at our disposal for cost reduction. We use the term "cost reduction" since we often want to minimize the value of a function of several independent variables. Such a reduction may be desired in order to either reach an optimum value, or to maximize another parameter of interest which increases with this decreasing cost. To name a few, Memetic algorithms, simulated annealing, force-directed placement, and evolution-based placement are some of the common algorithms that are used in cost reduction. The term "placement" broadly refers to the intermediate solution (or result) attained at an iterative step as the algorithm executes.

"Placement algorithms can be divided into two major classes: constructive placement and iterative improvement. In constructive placement, a method is used to build up a placement from scratch; in iterative improvement, algorithms start with an initial placement and repeatedly modify it in search of a cost reduction. If a modification results in a reduction in cost, the modification is accepted; otherwise it is rejected." [1]

These algorithms are heuristic in nature. That is, these algorithms produce a solution that is good enough for arriving to a solution to the problem at hand. There are parameters that are used for "tuning" these algorithms to arrive at a solution in the shortest possible time. These parameters are specific to individual algorithms that are used. Often, there is a tradeoff between the speed of execution of the algorithm and the accuracy of the result obtained.

There is a class of hybrid algorithms that combine evolutionary algorithms (evolutionbased placement algorithm listed above is a kind of evolutionary algorithm) and local searches and result in what we call as Memetic Algorithms.

"Memetic Algorithms are class of stochastic global search heuristics in which Evolutionary Algorithm based approaches are combined with problem-specific solvers. Later local search heuristics techniques are implemented. This hybridisation is to either accelerate or to discover good solution from the population where the evolution alone would take long time to discover or to reach the solution. Memetic 
Algorithms use heuristic local searches either approximate method or exact method to get the local refined solution from the population." [2]

Authors in [1] describe the force - directed placement with an analogy to the Hooke's law for the force exerted on stretched springs. While placing the modules, we assume that the modules are connected by a net which exerts an attractive force between them. The magnitude of this force is directly proportional to the distance between the modules. If the modules are allowed to move freely in the system, they would continue to move until they settle down at positions where there is a zero resultant force on each module and the system achieves a minimum energy state. "Hence, the force-directed placement methods are based on moving the modules in the direction of the total force exerted on them until this force is zero." [1] Authors have used the term "modules" for a specific application but it is equivalent to considering these modules as results of an optimization step.

Algorithms that fall in the genetic algorithm category have been inspired by the natural process of evolution. One such algorithm is the Simulated Evolution algorithm (SimE), which is a general search strategy for solving a variety of combinatorial optimization problems. It operates on a single solution, termed as population, and each population consists of elements. The algorithm has three basic steps in one main loop, namely, Evaluation, Selection, and Allocation. In the first step, the goodness of each element is measured as a single number between ' 0 ' and ' 1 ' which is indicative of how near the element is from its optimal solution. Following this step, Selection is carried out where unfit elements (elements that are far from their optimal solution) are selected in the current solution. It is because of Selection step that SimE does not get "trapped" at local minima since unfit elements are allowed to be a part of the intermediate solution. The last step is Allocation. The purpose here is to mutate the population by altering the current solution. This step has a high impact on the quality of the solution. [3]

For the purpose of this report, we will consider three algorithms that have been used for cost reduction optimizations. Namely, non-adaptive simulated annealing, adaptive simulated annealing, and random restart hill climbing will be evaluated in detail. The evaluation will cover the working of the algorithms, the pseudocode, MATLAB based 
implementations of these algorithms, and presentation of the performance comparison results from their implementation. 


\section{Non-adaptive simulated annealing}

\subsection{Overview}

Simulated annealing is a very time consuming algorithm but yields excellent results. The algorithm derives its name from metallurgy. Authors in [1] draw an interesting analogy between how the algorithm works and how metals are allowed to cool down so as to mold them in to the desired shape. A metal that is stressed has an imperfect crystal structure. How we bring about the metal to the desired form is to first heat the metal to a high temperature then cool it down gradually. In metallurgy, we refer to this as annealing.

At higher temperatures, the atoms in the metal have sufficient kinetic energy to break loose from their current incorrect positions. As the material cools down, the atoms slowly start getting trapped at the correct lattice positions. However, if we cool down the metal rather quickly, the atoms do not get a chance to get to the correct lattice positions and defects (due to the atoms being at the incorrect positions) become part of the crystal structure.

Simulated annealing algorithm does just that. In this algorithm, we start with an initial temperature and a starting configuration, or an initial guess for the solutions that would yield an optimal result. On successive iterations, we reduce the temperature and determine a configuration that results in an improvement over the current solution. We continue to reduce the temperature until we have reached a stopping temperature. The details on the working of this algorithm will be discussed in the following section.

It is imperative that the algorithm reaches the global minima of the function and does not get stuck at a local minima which may not yield the absolute minimum value, in our case, the cost. "Simulated annealing is a stochastic method to avoid getting stuck in local, nonglobal minima, when searching for global minima. This is done by accepting, in addition to transitions corresponding to a decrease in function value, transitions corresponding to an increase in function value. The latter is done in a limited way by means of a stochastic acceptance criterion. In the course of the minimization process, the probability of accepting deteriorations descends slowly towards zero." [4] 
For the purpose of demonstrating the working of this algorithm as implemented in MATLAB, we will work with a function which we can assume determines the cost in terms of the $x$ and $y$ values of the intermediate solution. The aim is in reaching the global minima of this function starting with some initial guess of the $x$ and $y$ independent variables. The intent of doing so is to demonstrate the working of this algorithm without focusing on the function itself since that may vary depending on the application. 


\subsection{Working of the non-adaptive simulated annealing algorithm}

We will look at the working of this algorithm over two sections. We present an abstract understanding of how the algorithm works here in this section. In the next section, we look at the pseudocode and detail how the algorithm proceeds to achieve the global minima of a given function.

To begin with, we start with an initial guess and a starting temperature for the working of this algorithm. Specific to our implementation, the initial guess is the $x$ and $y$ values of the intermediate solution (or the starting point). We also fix the starting temperature for the implementation of the algorithm. Once these are fixed, we set other parameters like cooling schedule, maximum consecutive rejections, stopping temperature, etc. We will discuss the relevance of each of these parameters in a following section.

The simulated annealing algorithm starts with accepting all moves but with a probability of accepting the move. At higher temperatures, the probability of accepting a move is higher. However, this probability decreases as the temperature decreases. The moves that cause a cost increase are accepted with a probability that decreases with the increase in cost.

In most implementations of this algorithm, the acceptance probability is given by exp($\Delta \mathrm{C} / \mathrm{T}$ ), where $\Delta \mathrm{C}$ is the cost increase. Initially, the temperature is set to a very high value so most of the moves are accepted. Acceptance (or rejection) of a move is determined by comparing the acceptance probability to a random probability value between 0 and 1 . At each iteration, the temperature is gradually reduced so the cost increasing moves are less likely to be accepted. Toward the end, only moves that cause a cost reduction are accepted and the algorithm converges to a low cost configuration. [1]

The fact that moves that result in a cost increase are also accepted (even though with a lower probability) ensures that the algorithm does not get "stuck" at a local minima and has a "fair" chance of covering all minima before reaching the global minima. 
It must be pointed out that we use the term "non-adaptive" with our application of the simulated annealing algorithm. This simply means that the cooling schedule is fixed. We do not vary the cooling schedule on successive iterations. In case of the adaptive simulated annealing algorithm, the cooling schedule will be adaptive, that is, it will vary as the algorithm executes. The adaptive version of the simulated annealing algorithm will be covered in the next section.

Following section will give the reader a better understanding of the working of this algorithm since we take the pseudocode of the algorithm in to account. 


\subsection{Pseudocode}

The simulated algorithm works as per the following pseudocode shown in Figure 2.3.1

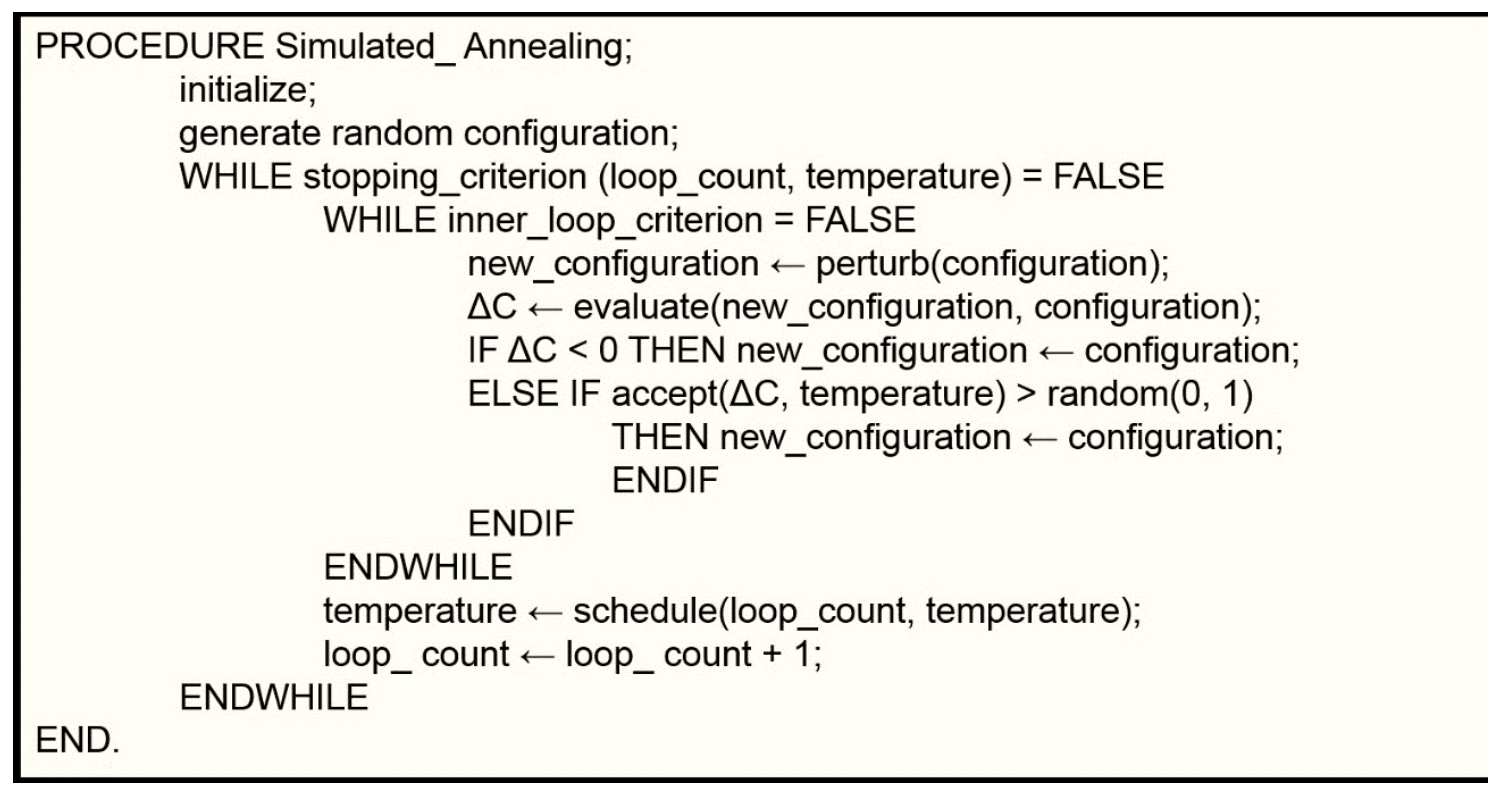

Figure 2.3.1: Pseudocode for the Simulated Annealing algorithm [1]

We will now analyze the algorithm in greater detail. We first start with the initialization using "initialize." The initialization involves setting the following parameters

1. Schedule: The cooling schedule. This determines the temperature decrements on successive iterations.

2. MaxConsecutiveRejections: Maximum number of consecutive rejections.

3. MaxSuccessAtTemperature: Maximum number of successful moves at a given temperature.

4. RandomGenerator: This generates a random configuration from an existing configuration.

5. InitialTemperature: The starting temperature for the simulated annealing algorithm.

6. StoppingTemperature: The stopping temperature for the simulated annealing algorithm. Algorithm exits implementation once this temperature has been reached. 
7. MaxTriesAtTemperature: Maximum number of moves that are permitted at a particular temperature.

8. StoppingValue: The stopping value for the simulated annealing algorithm. Algorithm exits implementation once this value of the function has been reached.

Following initialization, we generate a random configuration. In case of cost estimates, this can be computed using the function that determines the cost based on the $x$ and $y$ values of the solution. This is the "cost function." In our case, this value equals the initial cost which we wish to minimize.

Iterative improvements are carried out next. Until the temperature doesn't reach the stopping temperature or until we haven't reached the limit for maximum successive rejections as set by the MaxConsecutiveRejections parameter, we perform moves at each temperature. The number of moves at each temperature depends on parameters like MaxTriesAtTemperature and MaxSuccessAtTemperature.

We now consider a static situation when we are accepting or rejecting moves at a particular temperature. This implies that we have not reached the set limits of MaxTriesAtTemperature and MaxSuccessAtTemperature parameters. This is what we call the "inner loop criterion." The new configuration is reached by perturbing the existing configuration.

Perturbing the existing configuration is done in a two-step process. First, we generate random values for the $x$ and $y$ variables in the neighborhood of the current values. Next, we plug in these values in to the cost function that determines the cost based on the new values. The difference in costs in evaluated to determine if the new values result in a decrease, or an increase in the cost.

There are two scenarios that arise from the perturbation. If the cost decreases, then the move is accepted without any consideration to the acceptance probability given by exp($\Delta \mathrm{C} / \mathrm{T}$ ) where $\Delta \mathrm{C}$ is the cost increase and $\mathrm{T}$ is the current annealing temperature. However, if the cost increases, the move is still accepted with consideration to the 
acceptance probability. This is what makes the simulated annealing algorithm so special - it does not get stuck at a local minima.

Accepting (with or without consideration to the acceptance probability) or rejecting moves is done at a particular annealing temperature. Next, we reduce the temperature and follow the same procedure as in the previous step. The algorithm concludes execution if either the temperature is less than the stopping temperature or if the number of consecutive rejections has reached the preset. 


\subsection{Parameterization}

This section details the parameters we have used for the implementation of the Simulated Annealing algorithm. The parameters are generally tuned depending on the application.

1. Schedule: Since this is the non-adaptive simulated annealing algorithm, we use a fixed value for the cooling schedule. The temperature at the next step is 0.9 times the current temperature.

2. MaxConsecutiveRejections: Maximum number of consecutive rejections is set to 1,000 .

3. MaxSuccessAtTemperature: Maximum number of successful moves at a given temperature is set to 20 .

4. RandomGenerator: This generates a random configuration from an existing configuration. This is set to the anonymous function:

$@($ param $)(\text { param+(randperm }(\text { length }(\text { param }))==\text { length }(\text { param }))^{*}$ randn/100 $)$

Where param is a two-input vector with the current $x$ and $y$ values from the solution. The output is again a two member vector with the updated $x$ and $y$ values generated using randperm that generates random permutations of integers 0 and 1 . This is then multiplied by randn that generates normally distributed random number and is divided by 100 to keep the new $\mathrm{x}$ and $\mathrm{y}$ values within the neighborhood.

5. InitialTemperature: The starting temperature for the simulated annealing algorithm is set to 1

6. StoppingTemperature: The stopping temperature for the simulated annealing algorithm is set to $1 \mathrm{e}^{-8}$. Algorithm exits implementation once this temperature has been reached.

7. MaxTriesAtTemperature: Maximum number of moves that are permitted at a particular temperature is set to 300 . 
8. StoppingValue: The stopping value for the simulated annealing algorithm is set to 1. Algorithm exits implementation once this value of the function has been reached. In our application, this is a reasonable value for the minimum cost.

Appendix 1 includes the complete code for the non-adaptive simulated annealing algorithm. 


\subsection{Algorithm output}

The non-adaptive simulated algorithm is implemented using a sample cost function $2 x^{2}$ $-4 x y+y^{4}+2$. A 3-Dimensional graph of function $f$ shows that $f$ has two local minima at $(-1,-1,1)$ and $(1,1,1)$ and one saddle point at $(0,0,2)[5]$. This is shown in Figure 2.5.1.

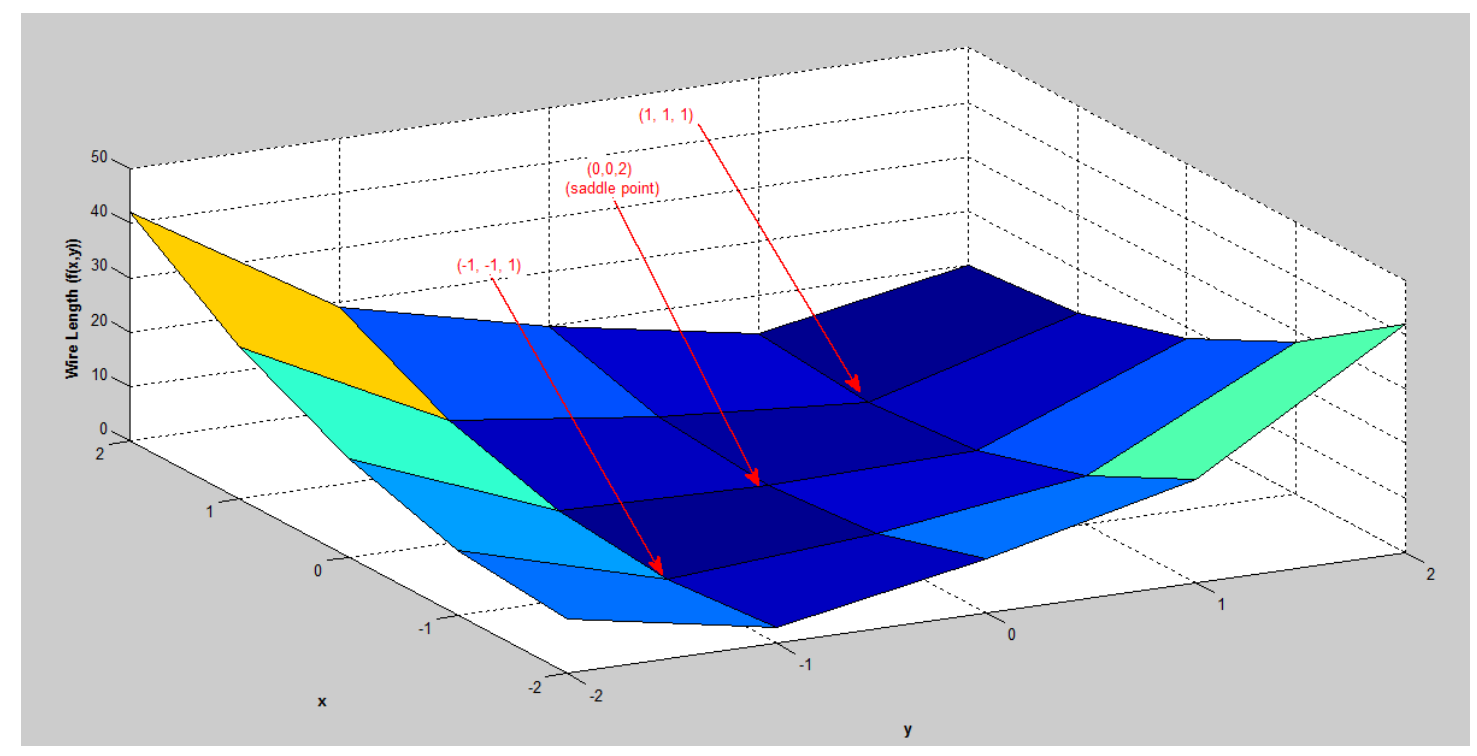

Figure 2.5.1: Cost function used for simulated annealing implementation [5]

We enter a vector as an initial guess and also a function handle so that the cost can be computed at subsequent iterations. The initial guess is a vector that contains the starting $x$ and $y$ values. This is shown in Figure 2.5.2

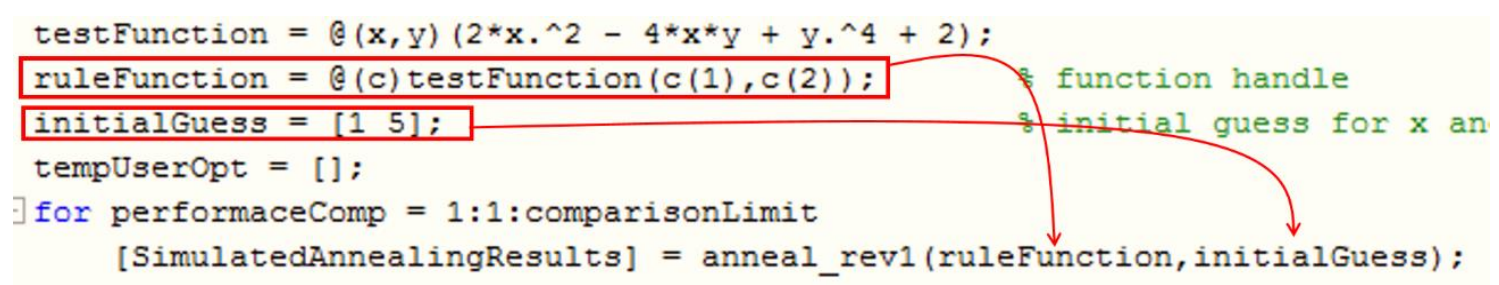

Figure 2.5.2: Passing the initial guess and the cost function handle in to the simulated annealing algorithm

Once the algorithm is run and it completes execution, we see that the solution converges to the minima (the minimum cost) and we get the optimal $x$ and $y$ values for the solution. 
The annealing algorithm waveforms at successive iterations and the final output are shown in Figures 2.5.3 and 2.5.4 respectively.

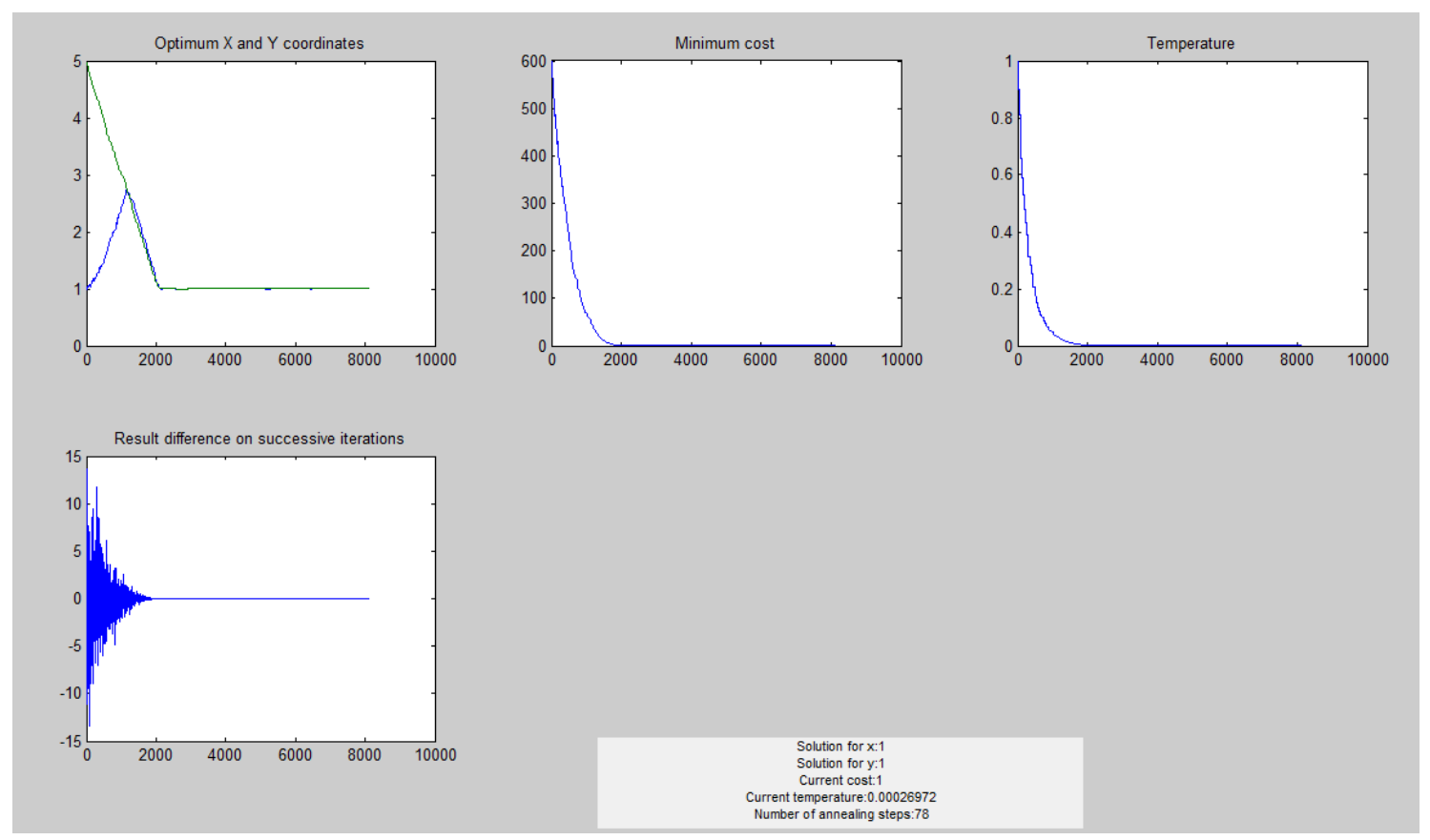

Figure 2.5.3: Simulated annealing algorithm waveforms at successive iterations

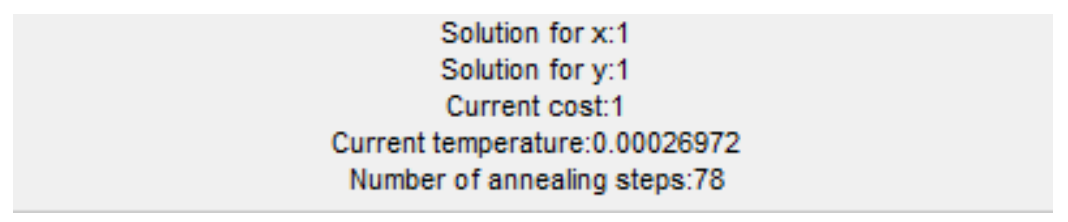

Figure 2.5.4: Output of the simulated annealing algorithm with the minimum cost and the $x$ and $y$ values for the solution

The results conform to the known minima for the given function. As is evident in Figure 2.5.4 the algorithm completes execution once the maximum number of consecutive rejections has been reached and not (in this case) because of having reached the minimum temperature. 


\section{Adaptive Simulated Annealing}

\subsection{Motivation}

Iterative improvement algorithms such as Simulated Annealing produce accurate results at the cost of enormous computation time. Such time-cost considerations encourage us to seek other algorithms that are more efficient. One such enhancement is the Adaptive Simulated Annealing (ASA) algorithm. ASA reduces the computation time required to reach a solution at the cost of some loss in accuracy of the solution.

Though at times it might not seem like a significant improvement in the computation time for relatively simple computations, the computation time is significantly improved for complex computations.

Another motivation in choosing the ASA is that we have the choice of going for a parameter-free simulated annealing algorithm. Such an advantage is crucial in that we can avoid having to deal with setting parameters which ultimately determine both the computation time and the accuracy of the results. This benefits the user with results that have much less dependence on the parameters, since just a few parameters are used for this form of ASA.

What makes ASA different from SA (Simulated Annealing) is that since ASA does not require the implementer to tune any parameters, a feedback mechanism is used to adjust the annealing temperature rather than using a fixed cooling schedule as in the case of SA. The parameters that are set and adjusted are the temperature and the acceptance rate. Over the subsequent sections, we will look in to the details of how these parameters are set, and the working of the ASA. 


\subsection{Working of the adaptive simulated annealing algorithm}

The Simulated Annealing (SA) algorithm works by reaching a state of thermal equilibrium to yield globally optimal solutions. This requires a series of annealing steps that cool down the temperature. Often, the cooling results in a prohibitively long computation time.

"Lam and Delosme proposed an approximate thermal equilibrium they call Dequilibrium which balances the trade-off of required computation time and the quality of the solution found by the run of SA. Under certain assumptions about the forms of the distribution of the cost values and the distribution of cost value changes, they analyzed their model and determined the annealing schedule that maintains the system in D-equilibrium (i.e., the annealing schedule that optimally balances the computational cost / solution quality trade-off). This "optimal" annealing schedule adjusts the temperature based on the parameter $\lambda$ which controls the cost-quality trade-off and more importantly based on the current rate of accepted moves. Analyzing their annealing schedule, Lam and Delosme determined that the temperature is reduced the quickest when the probability of accepting a move is equal to $0.44 . "[6]$

After having made this observation that a faster cooling rate led to a shorter annealing run, the size of the neighborhood considered for the moves was allowed to fluctuate to match this target move acceptance rate of 0.44 as closely as possible. The idea behind this is to either increase the acceptance rate by decreasing the maximum distance from the current state or to decrease the acceptance rate by increasing the maximum distance from the current state.

Swartz presented a modified version of the Lam and Delosme's annealing schedule. Instead of having a monotonically decreasing temperature, Swartz proposed controlling the temperature by continuously increasing and decreasing it on the basis of the acceptance rate. Starting with an initial acceptance rate of 1.0, the rate decreases exponentially during the first $15 \%$ of the run until it reaches 0.44 . Following this, it remains nearly constant for the next $50 \%$ of the run and then it exponentially decreases to 0 by the end of the run. 
Boyan presented an approach similar to Swartz's where a feedback mechanism was used to adapt the temperature in order to track the theoretical "optimal" acceptance rate. Doing so had the advantage of not having to modify the neighborhood function during the search. This made the "Modified Lam" annealing schedule problem - independent. [7]

Unlike the SA algorithm, we don't use the temperature as a stopping criterion. Instead, we use the maximum number of evaluations of the cost calculations as the criteria for stopping the algorithm. Note that this value may be changed by the user.

In the following section, we present the pseudocode for the ASA algorithm and run through the steps followed therein. 


\subsection{Pseudocode}

The simulated algorithm works as per the following pseudocode shown in Figure 3.3.1

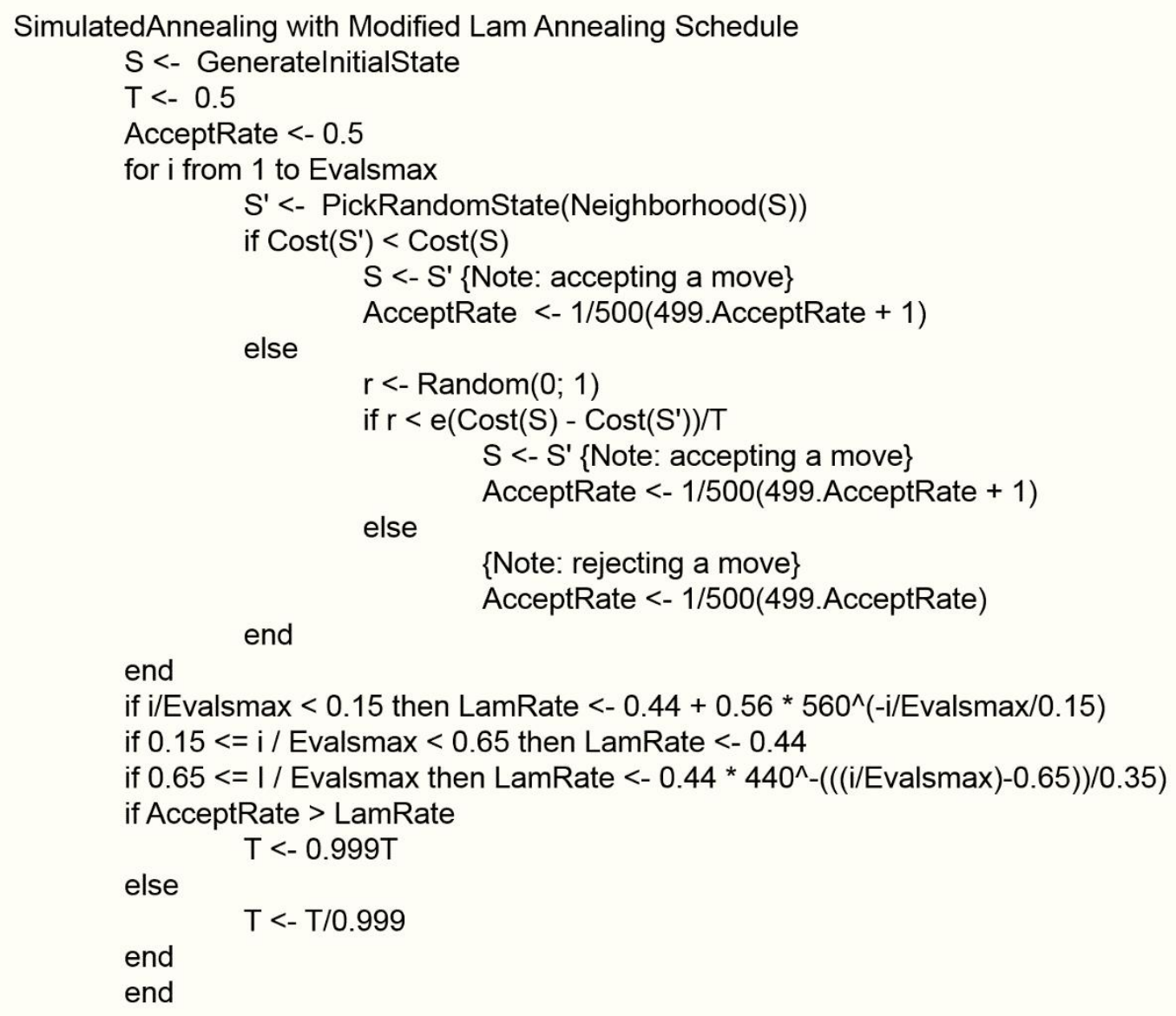

Figure 3.3.1: Pseudocode for the Adaptive Simulated Annealing algorithm [6]

Let us take a detailed look at how the algorithm is implemented. We begin with generating an initial state based on the initial $\mathrm{x}$ and $\mathrm{y}$ values provided to the function. Next, the initial temperature is set to a value of 0.5 and initial accept rate to 0.5 . We set a reasonably higher value for Evalsmax, i.e., the maximum number of evaluations that will be carried out.

Once inside the iterative loop, we carry out one iteration at a time until the number of operations has reached Evalsmax or until the algorithm reaches convergence. At each iteration, we choose a neighborhood value of $S$ (the current values) and evaluate the 
cost function, in our case the cost as a result of these neighborhood values. If the new cost is less than the current cost, accept the move with an increased acceptance rate. If the cost will increase as a result of this move, the move is accepted depending on the probability that decreases with increasing cost. However, if the cost increase is significant, the move is rejected. This is fairly similar to the SA algorithm.

What differentiates between the ASA from the SA algorithm is that we allow the current temperature to fluctuate based on the AcceptRate and the LamRate (LamRate is the "target" acceptance rate). As per the idea of the ASA, we try to stick to a probability of accepting moves to 0.44 so that the temperature reduces the fastest and results in a shorter convergence time. This is controlled by the part of the code shown in Figure 3.3.2.

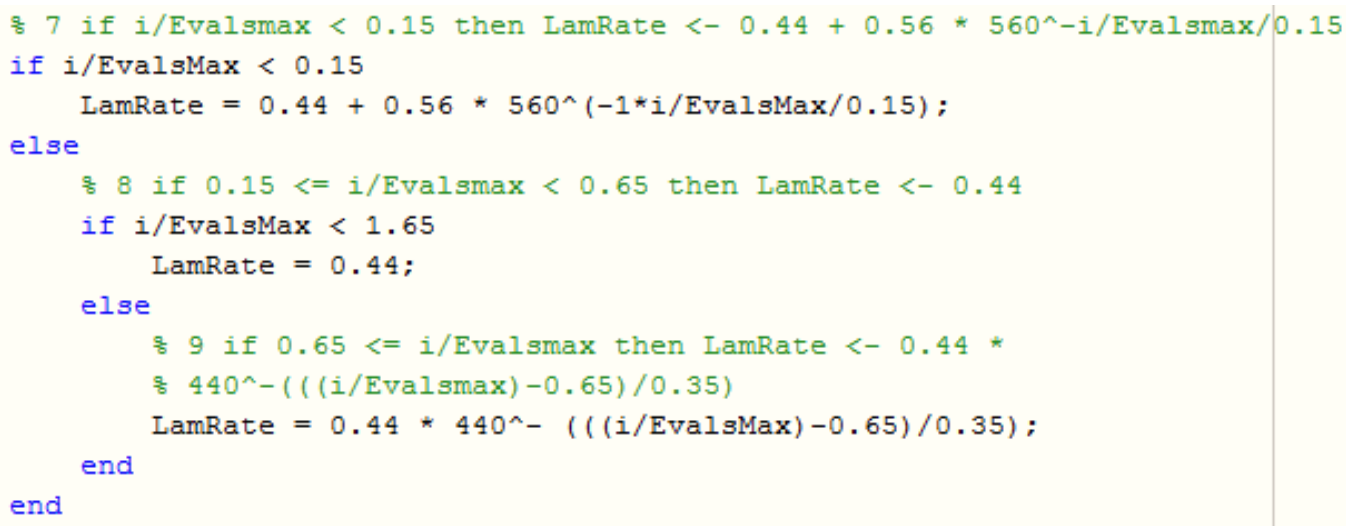

Figure 3.3.2: Controlling the temperature based on the LamRate and AcceptRate

Appendix 1 provides the complete MATLAB code for the Adaptive Simulated Annealing algorithm. 


\subsection{Algorithm output}

As in the case of the non-adaptive SA algorithm, we use the sample cost function $2 x^{2}-$ $4 x y+y^{4}+2$. A 3-Dimensional graph of function $f$ shows that $f$ has two local minima at $(-$ $1,-1,1)$ and $(1,1,1)$ and one saddle point at $(0,0,2)[5]$. This is shown in Figure 3.4.1.

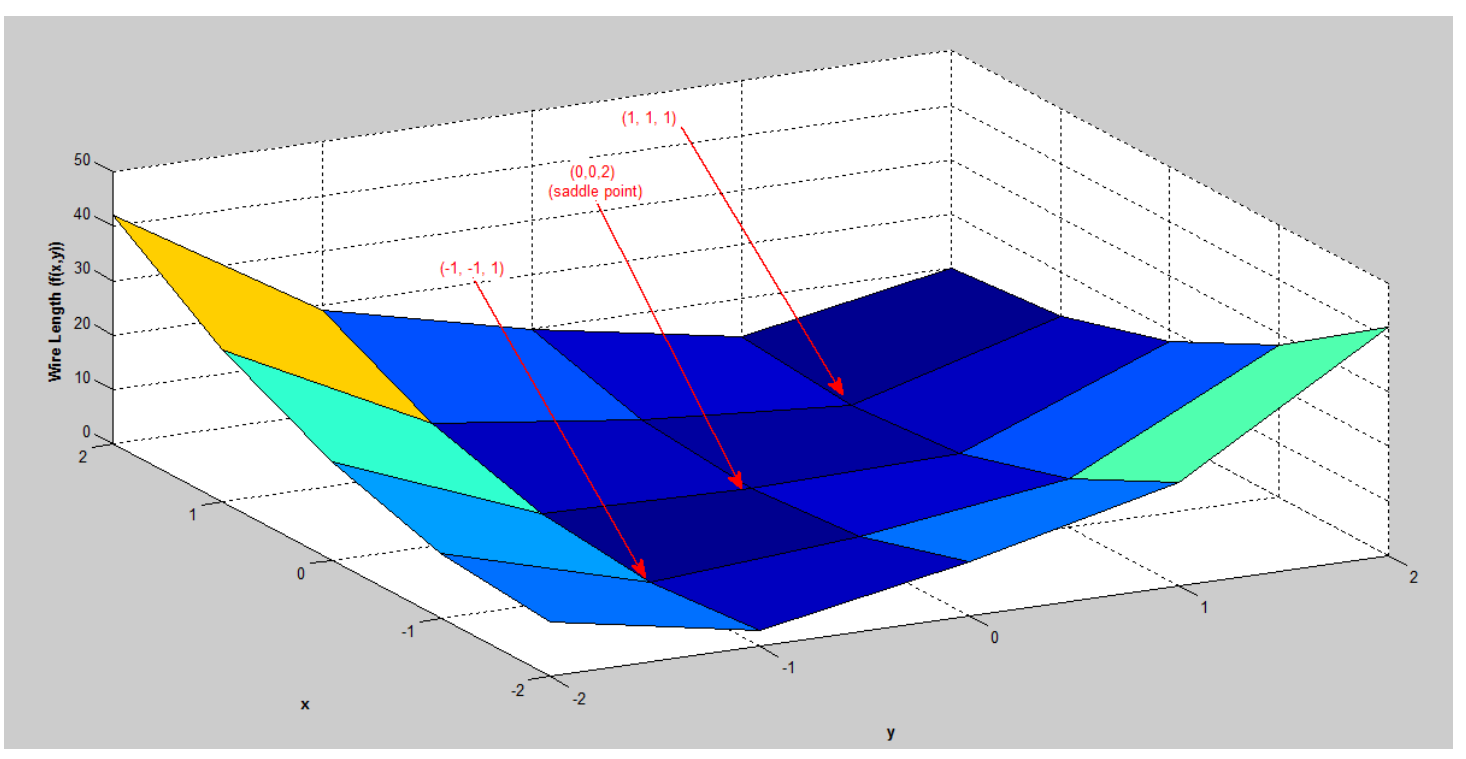

Figure 3.4.1: Cost function used for simulated annealing implementation [5]

We enter a vector as an initial guess and also a function handle so that the cost can be computed at subsequent iterations. The initial guess is a vector that contains the starting $x$ and $y$ values. This is shown in Figure 3.4.2

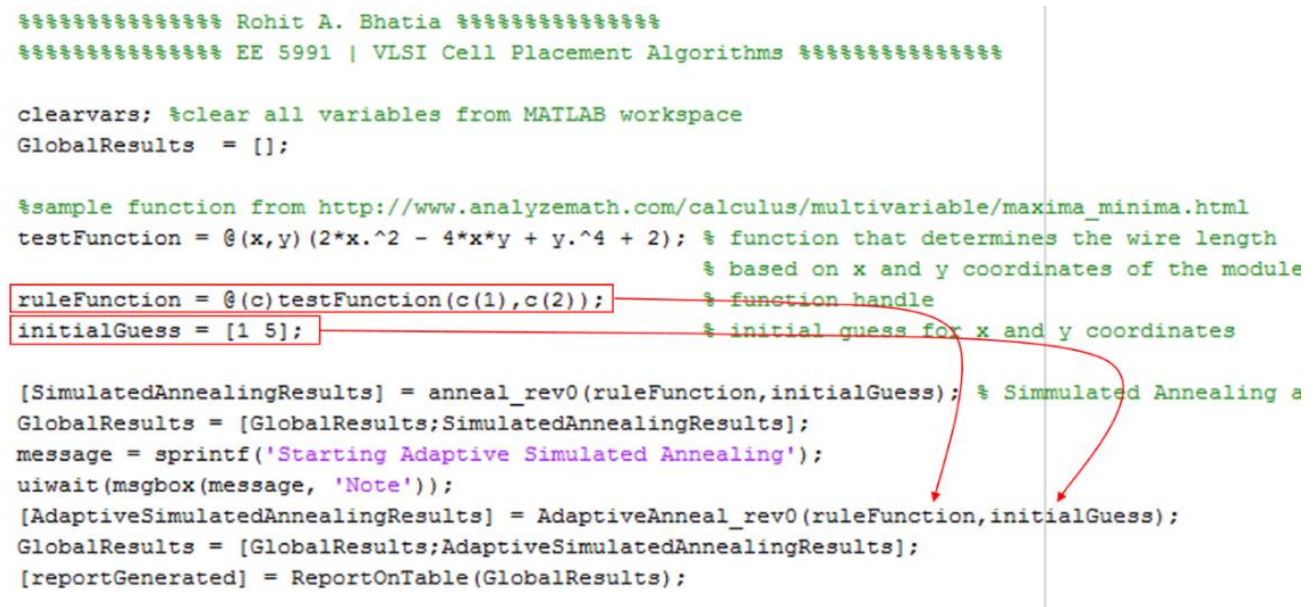

Figure 3.4.2: Passing the initial guess and the cost function handle in to the adaptive simulated annealing algorithm 
Once the algorithm is run and it completes execution, we see that the solution converges to the minima (the minimum cost) and we get the optimal $x$ and $y$ values for the solution. The annealing algorithm waveforms at successive iterations and the final output are shown in Figures 3.4.3 and 3.4.4 respectively.
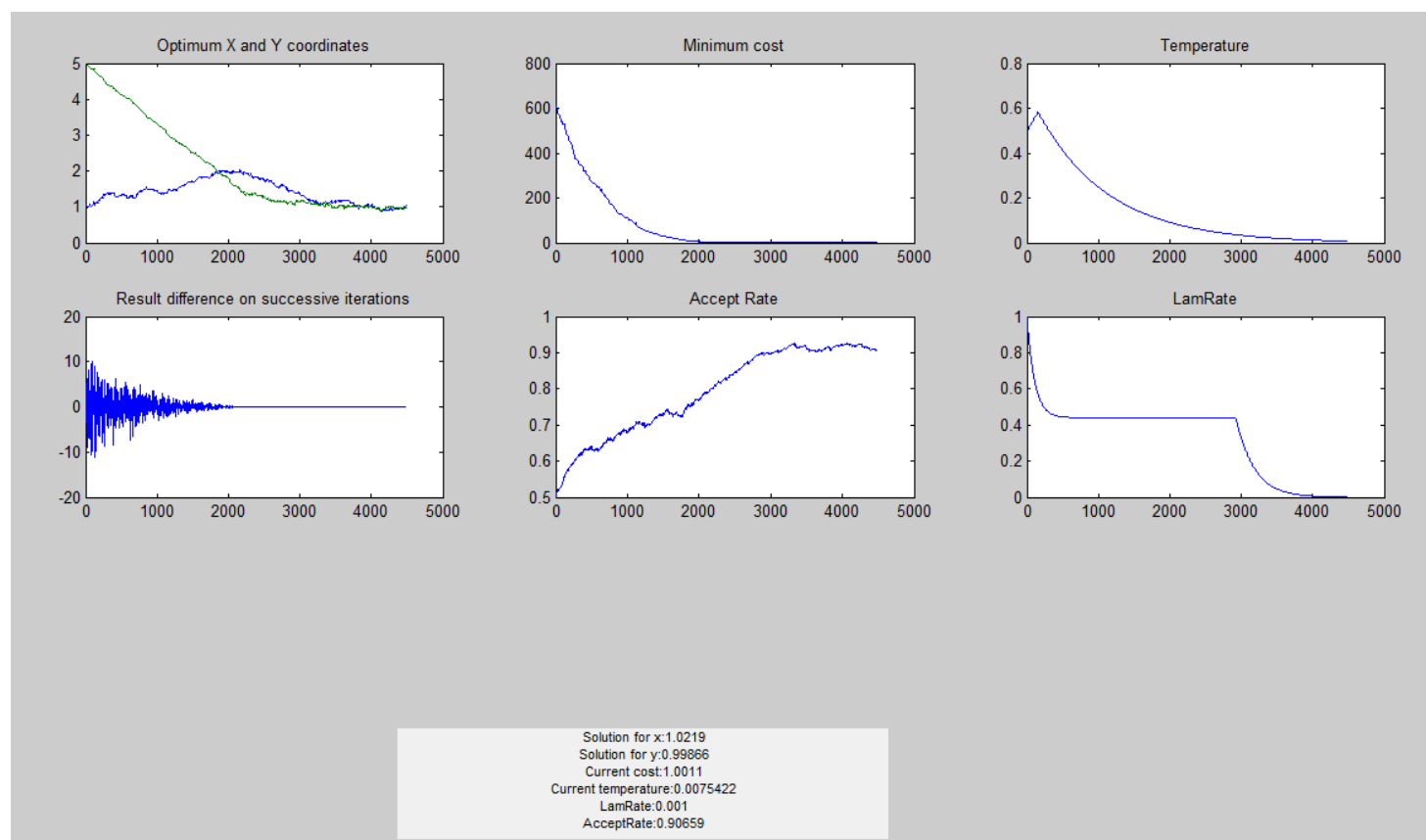

Figure 3.4.3: Adaptive Simulated Annealing algorithm waveforms at successive iterations

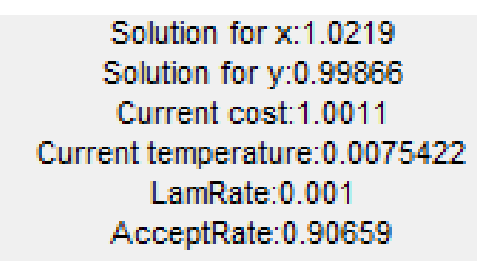

Figure 3.4.4: Output of the simulated annealing algorithm with the minimum cost and the $x$ and $y$ values for the solution

We notice from the results that the Adaptive Simulated Annealing algorithm converges reasonably faster than the SA algorithm at the cost of some accuracy in the results. This is the tradeoff that the user has to account for between the convergence time and the accuracy of the solution. Detailed performance comparisons are provided in a subsequent section. 


\section{Random restart hill climbing}

\subsection{Overview}

The classical hill climbing algorithm (also called gradient descent algorithm) is often used in finding the optimal points of a given function. In our case, the cost function is what is of interest to us for which we want to determine the optimum $x$ and $y$ values that yield the minimum cost.

Though simulated annealing and adaptive simulated annealing are the usual choice in seeking the minimum value of the cost function, the hill climbing algorithm with suitable enhancement(s) can also be employed to accomplish this task.

The classical hill climbing algorithm yields the optimum points of a function. Since we are interested in the global minima, the hill climbing algorithm with suitable enhancements is used to do so. But what is the need of an enhancement to this algorithm? The downside of the classical hill climbing algorithm is that it yields only local minima while we seek the global minima. Hence, the classical hill climbing algorithm will not work for this task.

Some of the variants of the hill climbing algorithm are the stochastic hill climbing [8] and the random restart hill climbing. In this implementation, the random restart hill climbing algorithm is used to find the optimum $x$ and $y$ values and thereby minimize the value of the cost function. Just like simulated annealing and adaptive simulated annealing, we start with an initial guess and iteratively move toward the optimal solution. In the following section, we will review the working of the adaptation made to the hill climbing algorithm. 


\subsection{Working of the random restart hill climbing algorithm}

Before proceeding with discussing how the random restart hill climbing algorithm works, it would be helpful to discuss how the classical hill climbing algorithm works. We start with a cost function (assume a function of two variables $f(x, y)$, referred to as $f$ ) and an initial starting point $x_{0}, y_{0}$.

First, we compute the gradient of $f$ at $x_{0}, y_{0}$ in terms of the partial derivatives. The gradient is given as

$$
\nabla f=\left(\frac{\partial f}{d x}\right) i+\left(\frac{\partial f}{\partial y}\right) j
$$

Where the $\partial$ terms are the partial derivatives of $f$ at the current $x$ and $y$ values and $i$ and $j$ are unit vectors along the $x$ and $y$ directions respectively. This implies that we first calculate the partial derivatives of $f$ and multiply those with $i$ and $j$ as in the equation above. Suppose now we fix our new points

$$
\begin{aligned}
& x^{i+1}=x^{i}+h \nabla f^{i} \\
& y^{i+1}=y^{i}+h \nabla f^{i}
\end{aligned}
$$

We now have the new (neighborhood) points only in terms of one unknown, namely $h$. The $\mathrm{h}$ is what we refer to as the step size. We now express $f^{+1}$ in terms of only one unknown h. Next, we take the derivate of the cost (or objective) function $f$ with respect to $h$ and get a function in terms of $h$ alone, say $r(h)$. To determine what should be the step size $\mathrm{h}$ so as to minimize the cost function, we only need to find the roots of $r(h)$ and plug the obtained value of $\mathrm{h}$ back in equation (2) to get the neighborhood points $x^{i+1}$ and $y^{i+1}$. If the roots are not real, we start off with a random initial condition and carry out iterative searches starting from that point.

Based on the new points, we follow the same procedure again until we reach a point where the gradient of the cost function is zero. This indicates that a critical point is reached; a point beyond which further improvisation is not possible. 
Having reached a critical point, we have implemented the classical form of the hill climbing algorithm. However, this may not necessarily be the global minima since there is a chance that a local minima could have been reached. This is not desirable since we seek a global minima.

Of the several alternatives available, we implement the RRHC (Random Restart Hill Climbing) algorithm. Once a local minima has been reached, do not let it terminate. Instead, we begin the search starting from a random initial condition ( $\left.\mathrm{x}_{0}{ }^{\prime}, \mathrm{y}_{0}{ }^{\prime}\right)$ and iteratively seek improvement by implementing another local search with this initial condition. Whenever a local minima is reached, the $\mathrm{x}$ and $\mathrm{y}$ values are stored along with the function value.

The procedure of starting with random initial conditions up on arrival at a local minima is carried out repeatedly until the preset number of maximum number of iterations is reached. Once the limit is reached, we look through the stored results and output the $x$ and $y$ values that provided us the least value of the cost function. This is the result of the random restart hill climbing algorithm. Appendix 1 provides the complete MATLAB code of the Random Restart Hill Climbing Algorithm. 


\subsection{Pseudocode}

The Random Restart Hill Climbing algorithm works as per the pseudocode shown in Figure 4.3.1

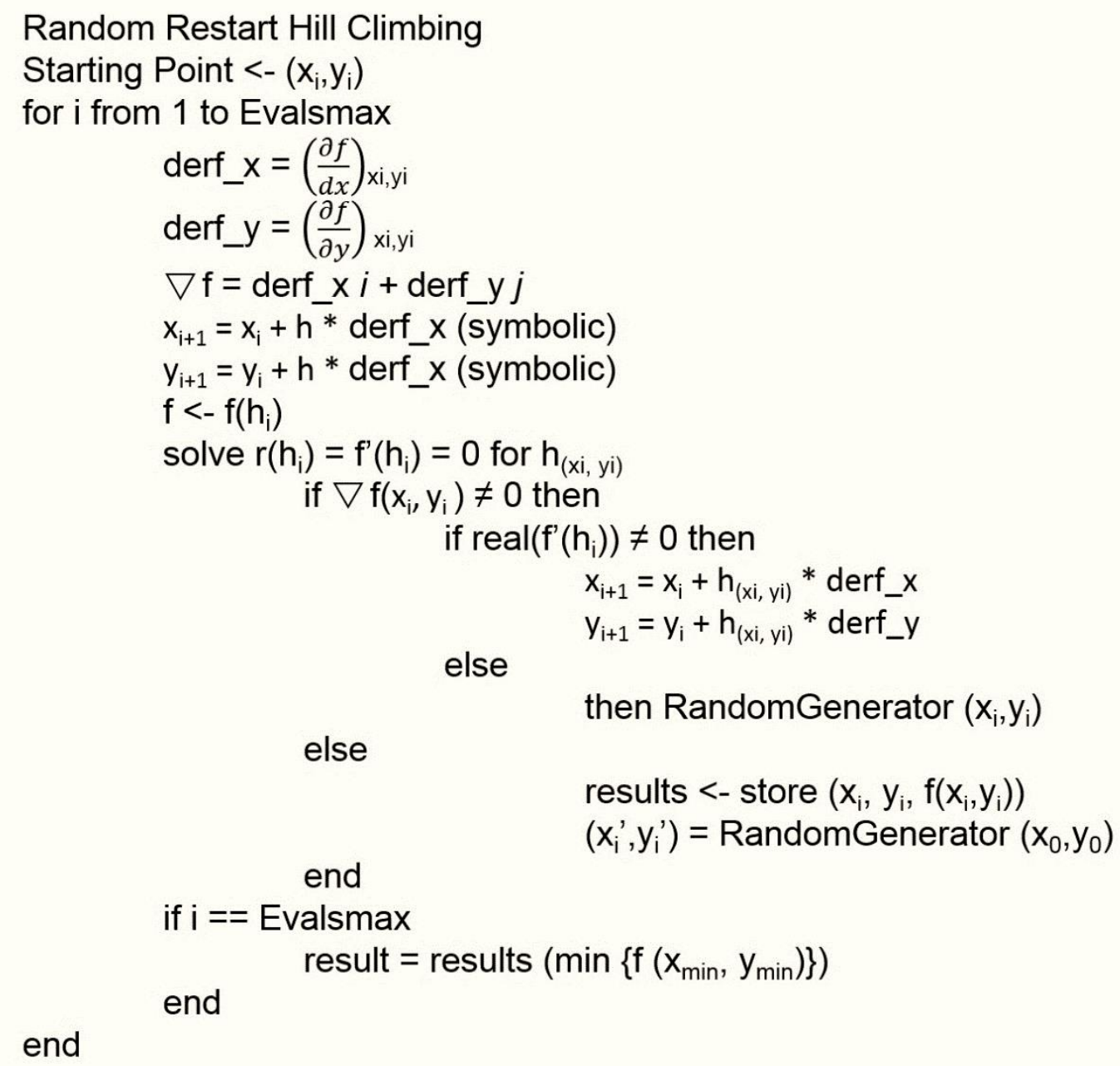

Figure 4.3.1: Pseudocode for the Random Restart Hill Climbing algorithm

Let us take a detailed look at how the algorithm is implemented. We begin with generating an initial state based on the initial $x$ and $y$ values provided to the function. Next, we symbolically get points $x_{i+1}$ and $y_{i+1}$ in terms of $h$, the step size and express the cost function in terms of $\mathrm{h}$ alone. It must be noted though that an approach other than symbolic differentiation might be required for cases where either the nature of the cost 
function is not known, or to account for the possibility of the cost function not being differentiable at all or not being differentiable at certain points of interest. We take the derivative of the cost function with respect to $h$ and solve it for $h$. Using this step size value, we compute the next iteration point $x_{i+1}=x_{i}+h \frac{\partial f}{d x}$ and $y_{i+1}=y_{i}+h \frac{\partial f}{\partial y}$. The same procedure is carried out until the gradient of $f \nabla f$ is not zero. If, however, $\nabla f$ is zero, the current $x$ and $y$ values are the critical points and we store these points, along with the value of $f$ at these points in a results table.

Since we reach a critical point when $\nabla f$ is zero, we want to ensure that this is indeed the global minima and not a local minima. To achieve this objective, we start the same iterative improvement from a randomly chosen point and see where this point takes us in terms of reaching the critical point. This is illustrated in Figure 4.3.2. Since the algorithm Hill Climbing algorithm is restarted from randomly chosen points, we call this the Random Restart Hill Climbing algorithm.

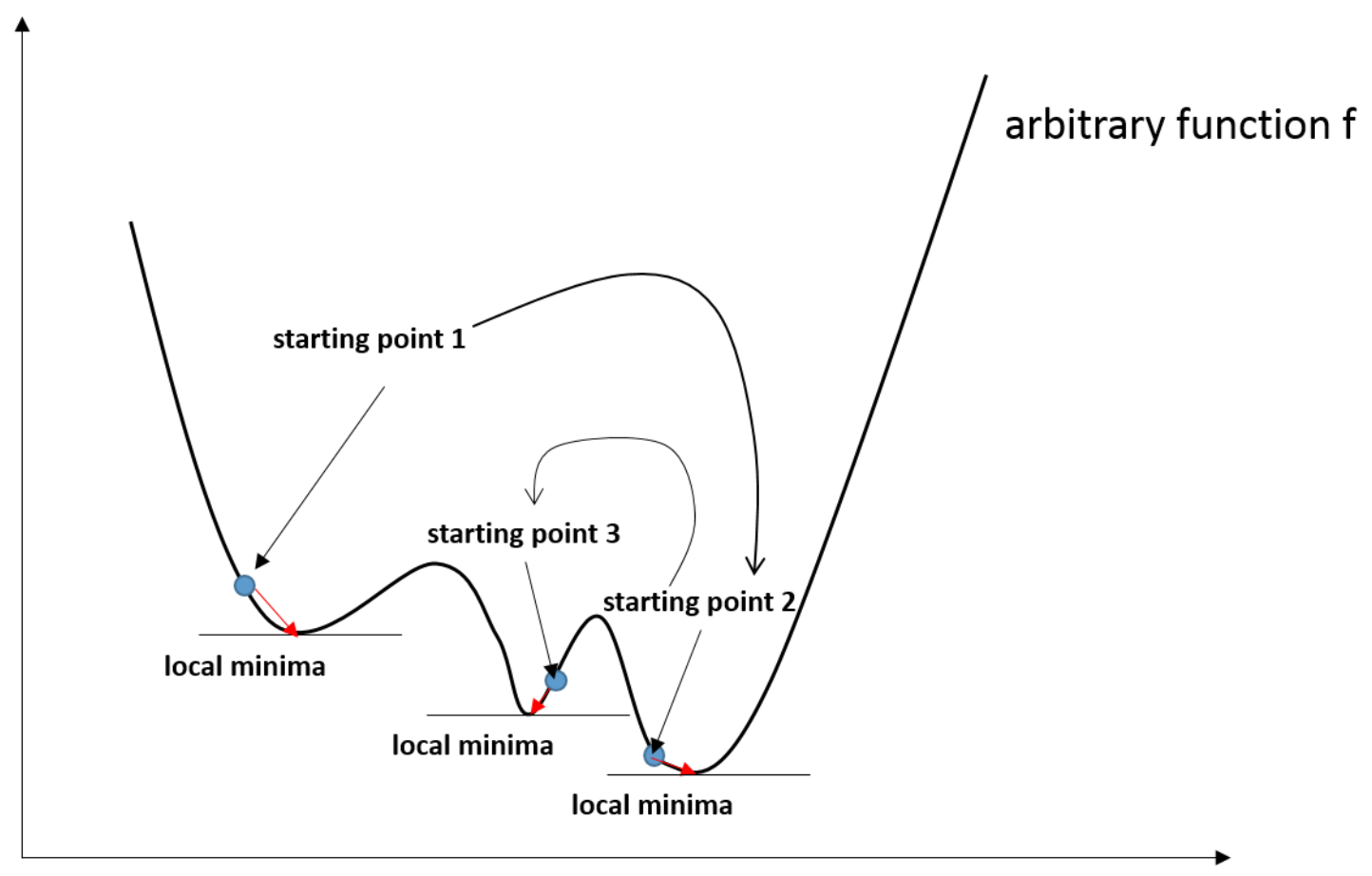

Figure 4.3.2: Random Restart of the Hill Climbing algorithm 
The procedure is repeated until the maximum number of iterations is reached. Once this limit is reached, we scan through the stored results and report the $\mathrm{x}$ and $\mathrm{y}$ values that yield the minimum value of the cost function. The limit is determined empirically. This becomes the result of the implementation of this algorithm. 


\subsection{Algorithm output}

As in the case of the previous algorithms, we use the sample cost function $2 x^{2}-4 x y+$ $y^{4}+2$. A 3-Dimensional graph of function $f$ shows that $f$ has two local minima at $(-1,-1,1)$ and $(1,1,1)$ and one saddle point at $(0,0,2)[5]$. This is shown in Figure 4.4.1.

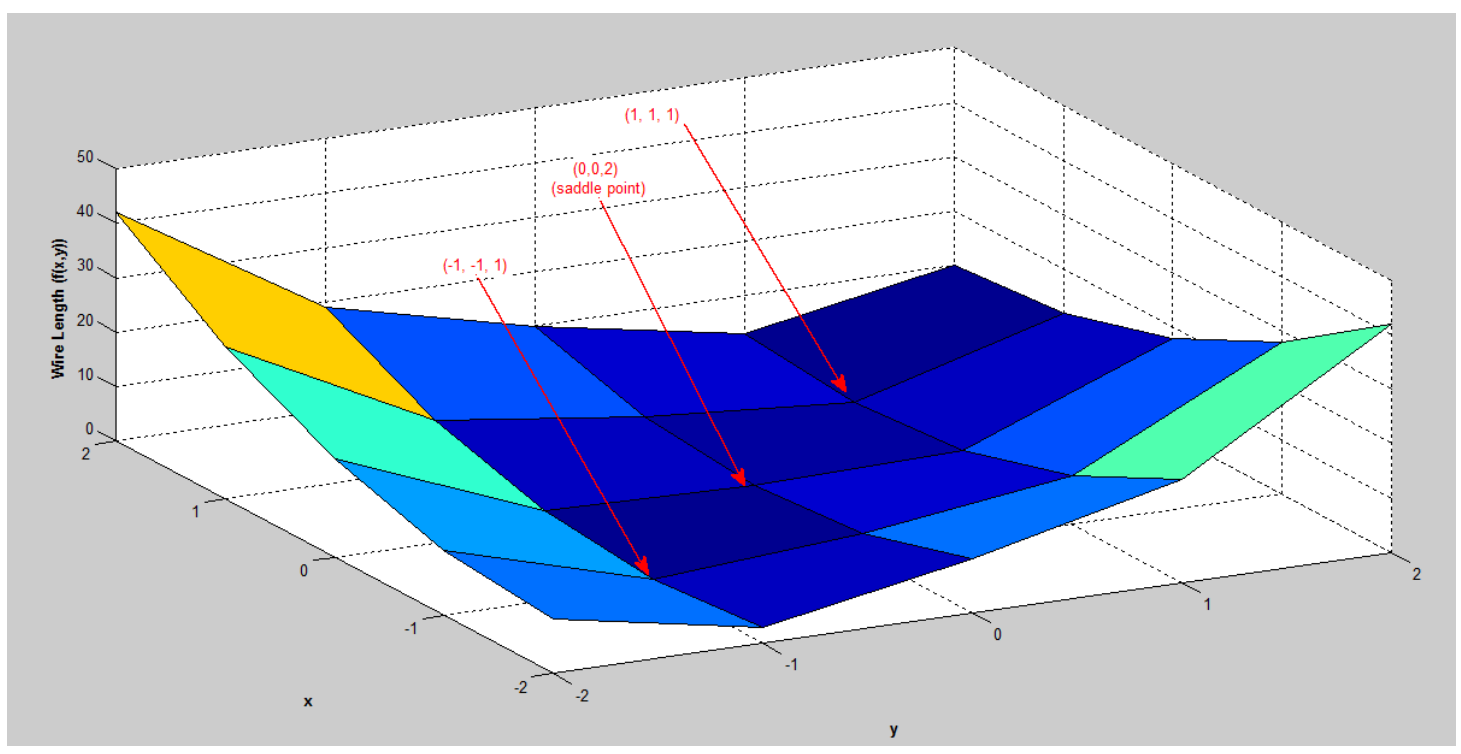

Figure 4.4.1: Cost function used for simulated annealing implementation [5]

We enter a vector as an initial guess and also the cost function so that the cost can be computed at subsequent iterations. The initial guess is a vector that contains the starting $x$ and $y$ values. This is shown in Figure 4.4.2

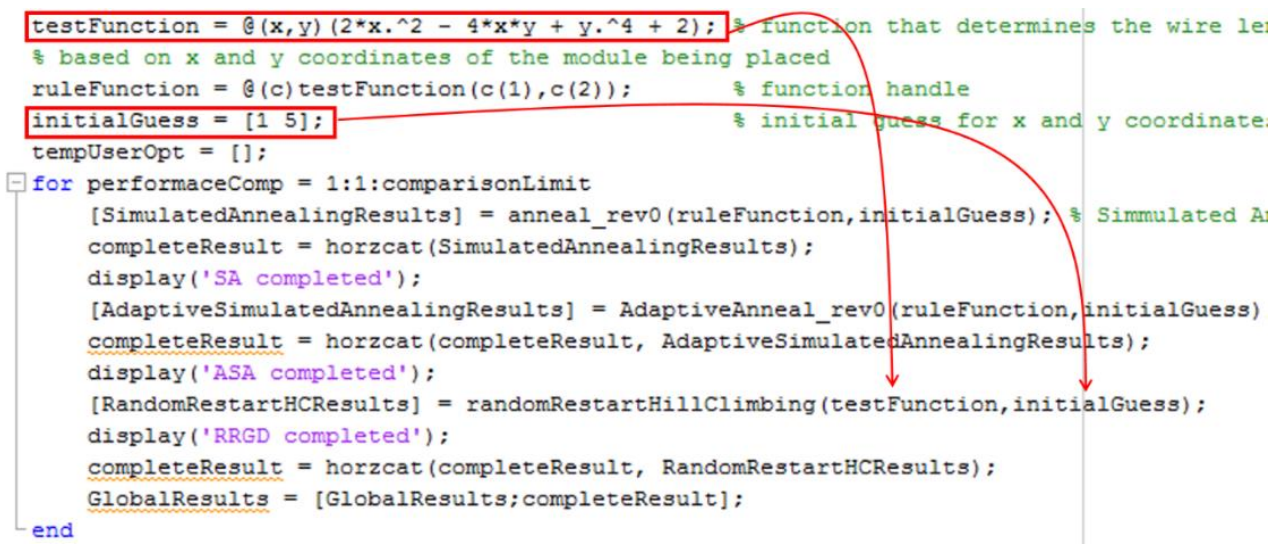

Figure 4.4.2: Passing the initial guess and the cost function in to the random restart hill climbing algorithm 
Once the algorithm is run and completes execution, we see a list of critical points. These points are of interest to us. Just before the algorithm terminates, we output the result based on the minimum value of the cost function. The random restart gradient descent algorithm waveforms at successive iterations and the final output are shown in Figures 4.4.3 and 4.4.4 respectively.

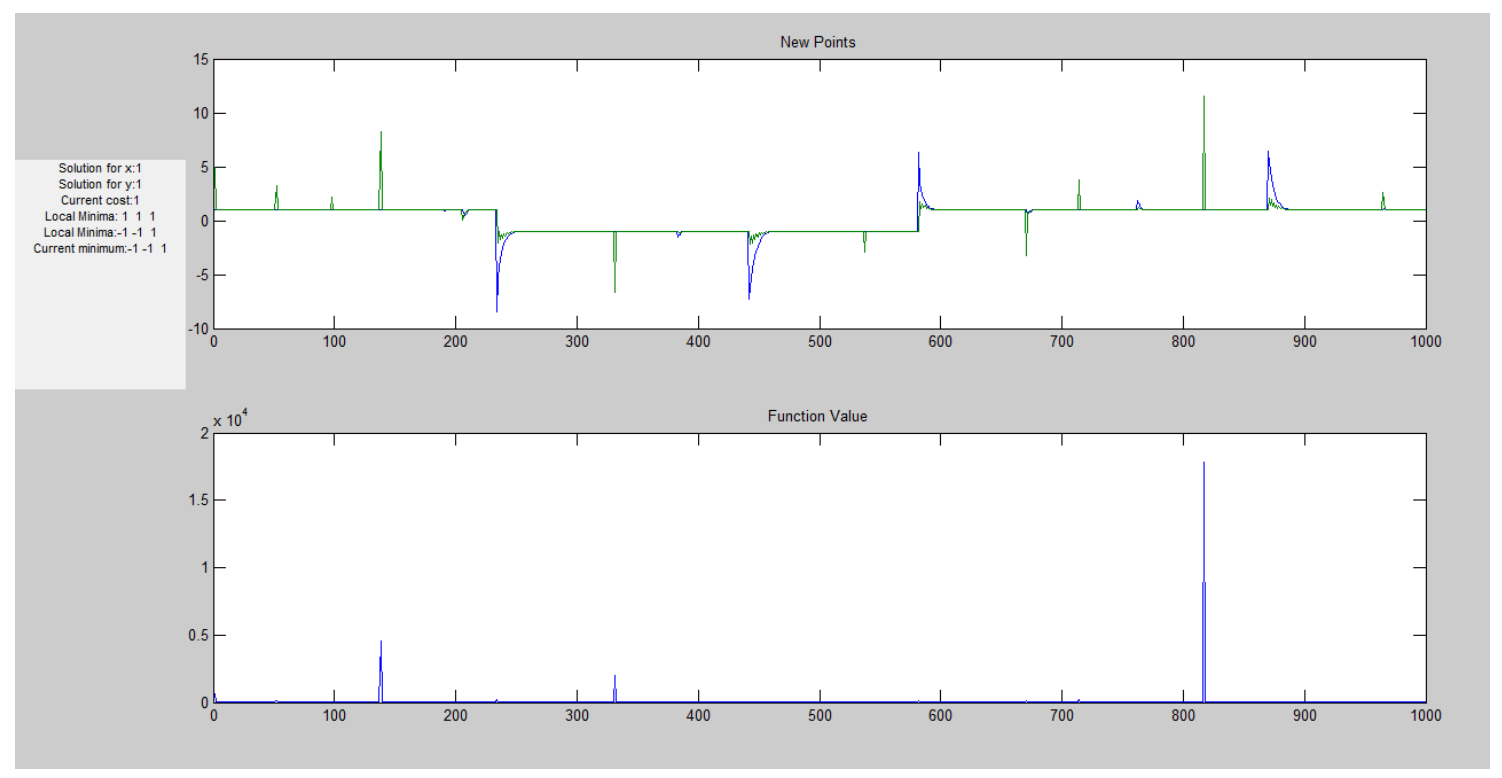

Figure 4.4.3: Random restart hill climbing algorithm waveforms at successive iterations

\author{
Solution for $\mathrm{x}: 1$ \\ Solution for $\mathrm{y}: 1$ \\ Current cost: 1 \\ Local Minima: 111 \\ Local Minima:-1 -11 \\ Current minimum:-1 $-1 \quad 1$
}

Figure 4.4.4: Output of the random restart hill climbing algorithm with the minimum cost and the $x$ and $y$ values for the solution

Since we have the set of results with local minima at $(-1,-1)$ and $(1,1)$ we can set additional filters so as to yield non-negative $x$ and $y$ values. Note that the third value in the results table indicates the value of the cost function at the given $x$ and $y$ values. 


\section{Performance comparison}

Based on the settings chosen for the three algorithms, each algorithm was tested for its performance with three different functions. We present a detailed performance comparison of these algorithms followed by a concluding section.

\subsection{Performance comparison with a cost function of two variables}

We use the sample cost function $2 x^{2}-4 x y+y^{4}+2$. The solution results for all the three algorithms are given in Tables 5.1.1, 5.1.2, and 5.1.3. The error is evaluated taking the known minimum function value as 1.0 .

\begin{tabular}{|c|c|c|c|c|}
\hline \multicolumn{5}{|c|}{ Simulated Annealing algorithm } \\
\hline & $\mathbf{x}$ & $\mathbf{y}$ & $\begin{array}{c}\text { minimum function } \\
\text { value }\end{array}$ & Error (\%) \\
\hline Trail 1 & 1.0000 & 1.0000 & 1.0000 & 0.0000 \\
\hline Trail 2 & 1.0000 & 1.0000 & 1.0000 & 0.0000 \\
\hline Trail 3 & 1.0000 & 1.0000 & 1.0000 & 0.0000 \\
\hline Trail 4 & 1.0000 & 1.0000 & 1.0000 & 0.0000 \\
\hline Trail 5 & 1.0000 & 1.0000 & 1.0000 & 0.0000 \\
\hline Trail 6 & 1.0000 & 1.0000 & 1.0000 & 0.0000 \\
\hline Trail 7 & 1.0000 & 1.0000 & 1.0000 & 0.0000 \\
\hline Trail 8 & 1.0000 & 1.0000 & 1.0000 & 0.0000 \\
\hline Trail 9 & 1.0000 & 1.0000 & 1.0000 & 0.0000 \\
\hline Trail 10 & 1.0000 & 1.0000 & 1.0000 & 0.0000 \\
\hline Average & 1.0000 & 1.0000 & 1.0000 & 0.0000 \\
\hline
\end{tabular}

Table 5.1.1: Solution from the SA algorithm for a cost function of two independent variables $x$, and $y$ 


\begin{tabular}{|c|c|c|c|c|}
\hline \multicolumn{5}{|c|}{ Adaptive Simulated Annealing algorithm } \\
\hline & $\mathbf{x}$ & $\mathbf{y}$ & $\begin{array}{c}\text { minimum function } \\
\text { value }\end{array}$ & Error (\%) \\
\hline Trail 1 & 1.0219 & 0.9987 & 1.0011 & 0.1100 \\
\hline Trail 2 & 1.1427 & 1.0316 & 1.0288 & 2.8800 \\
\hline Trail 3 & 1.0663 & 1.0176 & 1.0060 & 0.6000 \\
\hline Trail 4 & 1.0349 & 1.0619 & 1.0177 & 1.7700 \\
\hline Trail 5 & 1.1524 & 1.0736 & 1.0357 & 3.5700 \\
\hline Trail 6 & 1.0329 & 1.0047 & 1.0017 & 0.1700 \\
\hline Trail 7 & 0.9711 & 0.9577 & 1.0072 & 0.7200 \\
\hline Trail 8 & 1.0469 & 1.0101 & 1.0031 & 0.3100 \\
\hline Trail 9 & 0.9707 & 0.9977 & 1.0015 & 0.1500 \\
\hline Trail 10 & 0.9763 & 1.0202 & 1.0055 & 0.5500 \\
\hline Average & 1.0416 & 1.0174 & 1.0108 & 1.0800 \\
\hline
\end{tabular}

Table 5.1.2: Solution from the ASA algorithm for a cost function of two independent variables $\mathrm{x}$, and $y$

\begin{tabular}{|c|c|c|c|c|}
\hline \multicolumn{5}{|c|}{ Random Restart Hill Climbing algorithm } \\
\hline & $\mathbf{x}$ & $\mathbf{y}$ & minimum function value & Error (\%) \\
\hline Trail 1 & 1.0000 & 1.0000 & 1.0000 & 0.0000 \\
\hline Trail 2 & -1.0000 & -1.0000 & 1.0000 & 0.0000 \\
\hline Trail 3 & -1.0000 & -1.0000 & 1.0000 & 0.0000 \\
\hline Trail 4 & 1.0000 & 1.0000 & 1.0000 & 0.0000 \\
\hline Trail 5 & 1.0000 & 1.0000 & 1.0000 & 0.0000 \\
\hline Trail 6 & -1.0000 & -1.0000 & 1.0000 & 0.0000 \\
\hline Trail 7 & -1.0000 & -1.0000 & 1.0000 & 0.0000 \\
\hline Trail 8 & -1.0000 & -1.0000 & 1.0000 & 0.0000 \\
\hline Trail 9 & -1.0000 & -1.0000 & 1.0000 & 0.0000 \\
\hline Trail 10 & -1.0000 & -1.0000 & 1.0000 & 0.0000 \\
\hline Average & -0.4000 & -0.4000 & 1.0000 & 0.0000 \\
\hline
\end{tabular}

Table 5.1.3: Solution from the RRHC algorithm for a cost function of two independent variables $x$, and $y$ 
Figure 5.1.1 shows a plot of computation time of all the algorithms for 10 trials each.

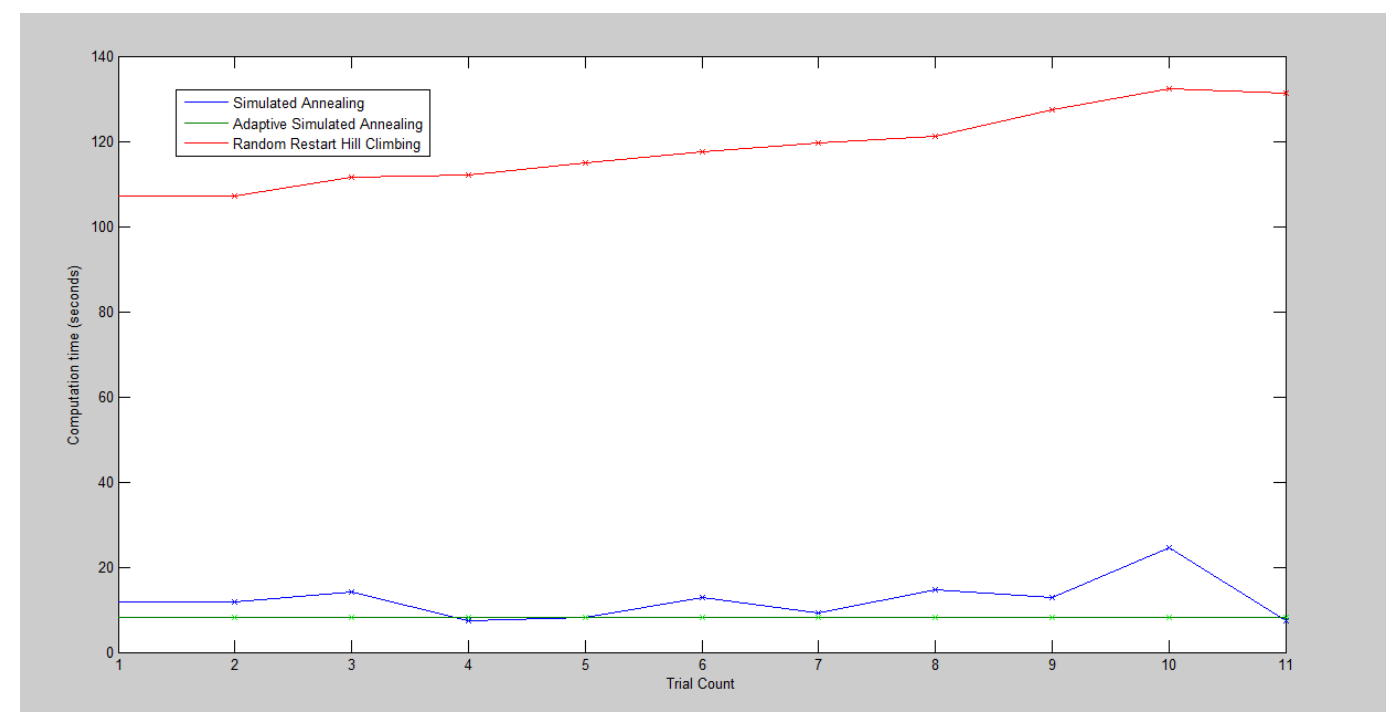

Figure 5.1.1: Computation time (sec.) for 10 tries of the three algorithms

Table 5.1.4 aims to provide information on the tradeoff between percentage error (hence accuracy) and computation time in each of the three algorithms

\begin{tabular}{|c|c|c|c|c|c|c|}
\hline & \multicolumn{2}{|c|}{ Simulated Annealing } & \multicolumn{2}{c|}{$\begin{array}{c}\text { Adaptive Simulated } \\
\text { Annealing }\end{array}$} & \multicolumn{2}{c|}{$\begin{array}{c}\text { Random Restart Hill } \\
\text { Climbing }\end{array}$} \\
\hline & Error (\%) & time (sec.) & Error (\%) & time(sec.) & Error (\%) & time(sec.) \\
\hline Trail 1 & 0.0000 & 11.8725 & 0.1100 & 8.3083 & 0.0000 & 107.0738 \\
\hline Trail 2 & 0.0000 & 14.2725 & 2.8800 & 8.2298 & 0.0000 & 111.5707 \\
\hline Trail 3 & 0.0000 & 7.4209 & 0.6000 & 8.1175 & 0.0000 & 111.9641 \\
\hline Trail 4 & 0.0000 & 8.1256 & 1.7700 & 8.1117 & 0.0000 & 114.8540 \\
\hline Trail 5 & 0.0000 & 12.8865 & 3.5700 & 8.0961 & 0.0000 & 117.4197 \\
\hline Trail 6 & 0.0000 & 9.1926 & 0.1700 & 8.1202 & 0.0000 & 119.5169 \\
\hline Trail 7 & 0.0000 & 14.5878 & 0.7200 & 8.0581 & 0.0000 & 121.1676 \\
\hline Trail 8 & 0.0000 & 12.8568 & 0.3100 & 8.2373 & 0.0000 & 127.4931 \\
\hline Trail 9 & 0.0000 & 24.6004 & 0.1500 & 8.0896 & 0.0000 & 132.2677 \\
\hline Trail 10 & 0.0000 & 7.5052 & 0.5500 & 8.2354 & 0.0000 & 119.4661 \\
\hline Average & 0.0000 & 12.3321 & 1.0830 & 8.1604 & 0.0000 & 118.2794 \\
\hline
\end{tabular}

Table 5.1.4: Computation time (sec.) vs. percentage error of the three algorithms for the given cost function of two independent variables 
Figure 5.1.2 shows a scatter plot of the computation time (sec.) vs. percentage error (hence accuracy) tradeoff in each of the three algorithms. The points with a glow represent the average computation time (sec.) vs. average error (\%) for each algorithm's implementation.

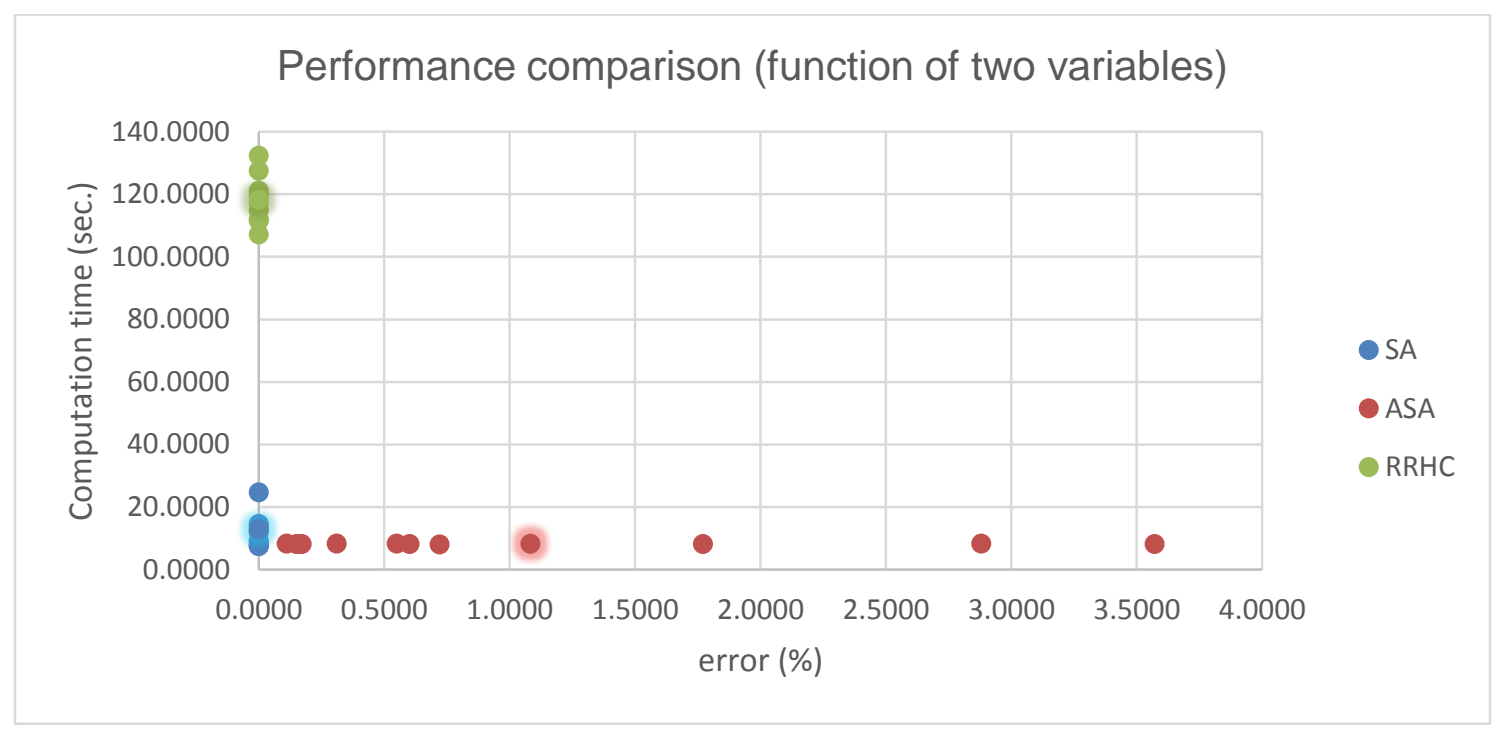

Figure 5.1.2: Computation time (sec.) vs. percentage error (hence accuracy) tradeoff in each of the three algorithms. 


\subsection{Performance comparison with a cost function of three variables}

We use the sample cost function $x^{2}+y^{2}+z^{2}$. The solution results for all the three algorithms are given in Tables 5.2.1, 5.2.2, and 5.2.3. The error is evaluated taking the known minimum function value as 0.0 since this function represents the volume of a sphere.

\begin{tabular}{|c|c|c|c|c|c|}
\hline & $\mathbf{x}$ & $\mathbf{y}$ & $\mathbf{z}$ & $\begin{array}{c}\text { Simulated Annealing algorithm } \\
\text { value }\end{array}$ & Error (\%) \\
\hline Trail 1 & $-4.30 \mathrm{e}-05$ & $5.32 \mathrm{e}-06$ & $5.48 \mathrm{e}-06$ & $1.91 \mathrm{e}-09$ & 0.0000 \\
\hline Trail 2 & $1.57 \mathrm{e}-07$ & $3.37 \mathrm{e}-05$ & $-8.28 \mathrm{e}-06$ & $1.21 \mathrm{e}-09$ & 0.0000 \\
\hline Trail 3 & $-1.98 \mathrm{e}-06$ & $2.07 \mathrm{e}-05$ & $3.58 \mathrm{e}-07$ & $4.35 \mathrm{e}-10$ & 0.0000 \\
\hline Trail 4 & $-4.80 \mathrm{e}-06$ & $-5.81 \mathrm{e}-06$ & $-3.33 \mathrm{e}-06$ & $6.78 \mathrm{e}-11$ & 0.0000 \\
\hline Trail 5 & $6.16 \mathrm{e}-06$ & $3.27 \mathrm{e}-05$ & $-4.77 \mathrm{e}-05$ & $3.38 \mathrm{e}-09$ & 0.0000 \\
\hline Trail 6 & $-2.26 \mathrm{e}-05$ & $-4.74 \mathrm{e}-06$ & $9.48 \mathrm{e}-05$ & $9.52 \mathrm{e}-09$ & 0.0000 \\
\hline Trail 7 & $5.79 \mathrm{e}-06$ & $2.47 \mathrm{e}-06$ & $-1.03 \mathrm{e}-06$ & $4.06 \mathrm{e}-11$ & 0.0000 \\
\hline Trail 8 & $3.85 \mathrm{e}-05$ & $4.83 \mathrm{e}-06$ & $4.49 \mathrm{e}-07$ & $1.50 \mathrm{e}-09$ & 0.0000 \\
\hline Trail 9 & $4.69 \mathrm{e}-08$ & $5.23 \mathrm{e}-06$ & $4.43 \mathrm{e}-05$ & $1.99 \mathrm{e}-09$ & 0.0000 \\
\hline Trail 10 & $2.91 \mathrm{e}-07$ & $-5.75 \mathrm{e}-07$ & $-5.95 \mathrm{e}-05$ & $3.54 \mathrm{e}-09$ & 0.0000 \\
\hline Average & $-2.15 \mathrm{e}-06$ & $9.39 \mathrm{e}-06$ & $2.56 \mathrm{e}-06$ & $2.36 \mathrm{e}-09$ & 0.0000 \\
\hline
\end{tabular}

Table 5.2.1: Solution from the SA algorithm for a cost function of three independent variables $x$, $y$, and $z$ 


\begin{tabular}{|c|c|c|c|c|c|}
\hline \multicolumn{7}{|c|}{ Adaptive Simulated Annealing algorithm } \\
\hline & $\mathbf{x}$ & $\mathbf{y}$ & $\mathbf{z}$ & $\begin{array}{c}\text { minimum function } \\
\text { value }\end{array}$ & Error (\%) \\
\hline Trail 1 & -0.0122 & 0.0897 & 0.1514 & 0.0311 & 3.1100 \\
\hline Trail 2 & 0.0166 & 0.3772 & 0.0833 & 0.1495 & 14.9500 \\
\hline Trail 3 & 0.0927 & 0.2953 & 0.0348 & 0.0970 & 9.7000 \\
\hline Trail 4 & 0.0447 & 0.0673 & -0.0234 & 0.0071 & 0.7100 \\
\hline Trail 5 & -0.0142 & 0.2292 & 0.0474 & 0.0550 & 5.5000 \\
\hline Trail 6 & -0.0395 & 0.2485 & 0.0017 & 0.0633 & 6.3300 \\
\hline Trail 7 & -0.0046 & -0.0289 & -0.0005 & 0.0009 & 0.0857 \\
\hline Trail 8 & 0.0796 & 0.6125 & 0.0352 & 0.3827 & 38.2700 \\
\hline Trail 9 & 0.0269 & 0.1638 & -0.0313 & 0.0285 & 2.8500 \\
\hline Trail 10 & -0.0528 & 0.4458 & 0.1669 & 0.2293 & 22.9300 \\
\hline Average & 0.0137 & 0.2500 & 0.0465 & 0.1044 & 10.4400 \\
\hline
\end{tabular}

Table 5.2.2: Solution from the ASA algorithm for a cost function of three independent variables $x, y$, and $z$

\begin{tabular}{|c|c|c|c|c|c|}
\hline \multicolumn{7}{|c|}{ Random Restart Hill Climbing algorithm } \\
\hline & $\mathbf{x}$ & $\mathbf{y}$ & $\mathbf{z}$ & $\begin{array}{c}\text { minimum function } \\
\text { value }\end{array}$ & Error (\%) \\
\hline Trail 1 & 0.0000 & 0.0000 & 0.0000 & 0.0000 & 0.0000 \\
\hline Trail 2 & 0.0000 & 0.0000 & 0.0000 & 0.0000 & 0.0000 \\
\hline Trail 3 & 0.0000 & 0.0000 & 0.0000 & 0.0000 & 0.0000 \\
\hline Trail 4 & 0.0000 & 0.0000 & 0.0000 & 0.0000 & 0.0000 \\
\hline Trail 5 & 0.0000 & 0.0000 & 0.0000 & 0.0000 & 0.0000 \\
\hline Trail 6 & 0.0000 & 0.0000 & 0.0000 & 0.0000 & 0.0000 \\
\hline Trail 7 & 0.0000 & 0.0000 & 0.0000 & 0.0000 & 0.0000 \\
\hline Trail 8 & 0.0000 & 0.0000 & 0.0000 & 0.0000 & 0.0000 \\
\hline Trail 9 & 0.0000 & 0.0000 & 0.0000 & 0.0000 & 0.0000 \\
\hline Trail 10 & 0.0000 & 0.0000 & 0.0000 & 0.0000 & 0.0000 \\
\hline Average & 0.0000 & 0.0000 & 0.0000 & 0.0000 & 0.0000 \\
\hline
\end{tabular}

Table 5.2.3: Solution from the RRHC algorithm for a cost function of three independent variables $x, y$, and $z$ 
Figure 5.2.1 shows a plot of computation time of all the algorithms for 10 trials each.

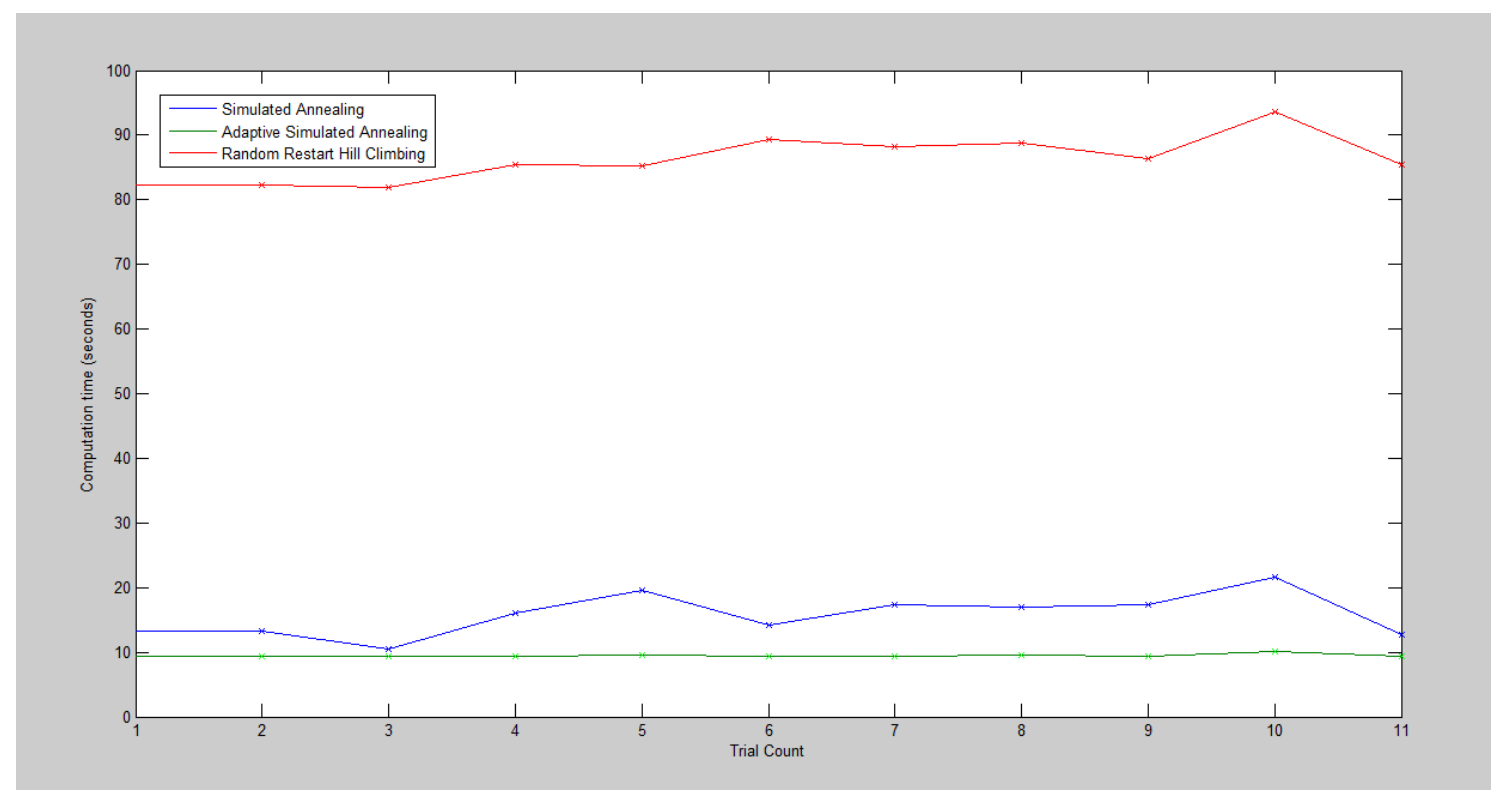

Figure 5.2.1: Computation time (sec.) for 10 tries of the three algorithms

Table 5.2.4 aims to provide information on the tradeoff between percentage error (hence accuracy) and computation time in each of the three algorithms.

\begin{tabular}{|c|c|c|c|c|c|c|}
\hline & \multicolumn{2}{|c|}{$\begin{array}{c}\text { Simulated Annealing } \\
\end{array}$} & \multicolumn{2}{c|}{$\begin{array}{c}\text { Adaptive Simulated } \\
\text { Annealing }\end{array}$} & \multicolumn{2}{c|}{$\begin{array}{c}\text { Random Restart Hill } \\
\text { Climbing }\end{array}$} \\
\hline & Error (\%) & time(sec.) & Error (\%) & time(sec.) & Error (\%) & time(sec.) \\
\hline Trail 1 & 0.0000 & 13.2846 & 3.1100 & 9.3280 & 0.0000 & 82.3744 \\
\hline Trail 2 & 0.0000 & 10.4691 & 14.9500 & 9.2858 & 0.0000 & 81.9271 \\
\hline Trail 3 & 0.0000 & 16.0278 & 9.7000 & 9.2807 & 0.0000 & 85.4474 \\
\hline Trail 4 & 0.0000 & 19.6527 & 0.7100 & 9.4716 & 0.0000 & 85.2702 \\
\hline Trail 5 & 0.0000 & 14.2018 & 5.5000 & 9.4105 & 0.0000 & 89.2661 \\
\hline Trail 6 & 0.0000 & 17.2582 & 6.3300 & 9.3741 & 0.0000 & 88.1859 \\
\hline Trail 7 & 0.0000 & 16.8966 & 0.0857 & 9.5158 & 0.0000 & 88.7392 \\
\hline Trail 8 & 0.0000 & 17.2577 & 38.2700 & 9.4201 & 0.0000 & 86.2815 \\
\hline Trail 9 & 0.0000 & 21.5238 & 2.8500 & 10.0450 & 0.0000 & 93.5806 \\
\hline Trail 10 & 0.0000 & 12.6871 & 22.9300 & 9.3865 & 0.0000 & 85.3771 \\
\hline Average & 0.0000 & 15.9259 & 10.4436 & 9.4518 & 0.0000 & 86.6450 \\
\hline
\end{tabular}

Table 5.2.4: Computation time (sec.) vs. percentage error of the three algorithms for the given cost function of three independent variables 
Figure 5.2.2 shows a scatter plot of the computation time (sec.) vs. percentage error (hence accuracy) tradeoff in each of the three algorithms. The points with a glow represent the average computation time (sec.) vs. average error (\%) for each algorithm's implementation.

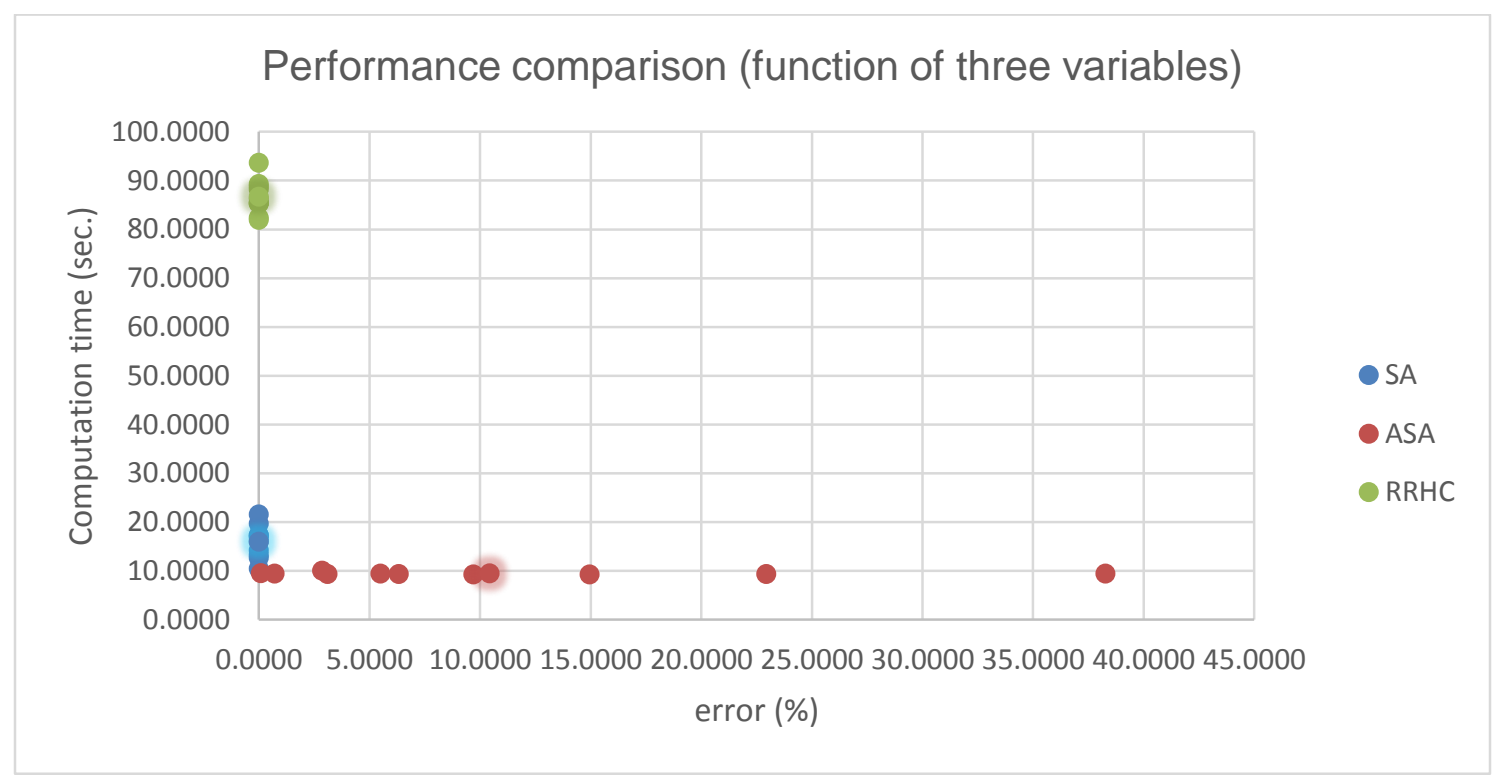

Figure 5.2.2: Computation time (sec.) vs. percentage error (hence accuracy) tradeoff in each of the three algorithms. 


\subsection{Performance comparison with a cost function of four variables}

We use the sample cost function $\left(2 w^{2}-4 w x+x^{4}+2\right)^{\star}\left(2 y^{2}-4 y z+z^{4}+2\right)$. The solution results for all the three algorithms are given in Tables 5.3.1, 5.3.2, and 5.3.3. The error is evaluated taking the known minimum function value as 1.0 .

\begin{tabular}{|c|c|c|c|c|c|c|}
\hline & $\mathbf{w}$ & $\mathbf{x}$ & $\mathbf{y}$ & $\mathbf{z}$ & $\begin{array}{c}\text { minimum function } \\
\text { value }\end{array}$ & Error (\%) \\
\hline Trail 1 & 1.0000 & 1.0000 & -1.0000 & -1.0000 & 1.0000 & 0.0000 \\
\hline Trail 2 & 1.0000 & 1.0000 & -1.0000 & -1.0000 & 1.0000 & 0.0000 \\
\hline Trail 3 & 1.0000 & 1.0000 & -1.0000 & -1.0000 & 1.0000 & 0.0000 \\
\hline Trail 4 & 1.0000 & 1.0000 & -1.0000 & -1.0000 & 1.0000 & 0.0000 \\
\hline Trail 5 & 1.0000 & 1.0000 & -1.0000 & -1.0000 & 1.0000 & 0.0000 \\
\hline Trail 6 & 1.0000 & 1.0000 & -1.0000 & -1.0000 & 1.0000 & 0.0000 \\
\hline Trail 7 & 1.0000 & 1.0000 & -1.0000 & -1.0000 & 1.0000 & 0.0000 \\
\hline Trail 8 & 1.0000 & 1.0000 & -1.0000 & -1.0000 & 1.0000 & 0.0000 \\
\hline Trail 9 & 1.0000 & 1.0000 & -1.0000 & -1.0000 & 1.0000 & 0.0000 \\
\hline Trail 10 & 1.0000 & 1.0000 & -1.0000 & -1.0000 & 1.0000 & 0.0000 \\
\hline Average & 1.0000 & 1.0000 & -1.0000 & -1.0000 & 1.0000 & 0.0000 \\
\hline
\end{tabular}

Table 5.3.1: Solution from the SA algorithm for a cost function of four independent variables $w$, $\mathrm{x}, \mathrm{y}$, and $\mathrm{z}$ 


\begin{tabular}{|c|c|c|c|c|c|c|}
\hline \multicolumn{7}{|c|}{ Adaptive Simulated Annealing algorithm } \\
\hline & w & $\mathbf{x}$ & $\mathbf{y}$ & $\mathbf{z}$ & $\begin{array}{c}\text { minimum function } \\
\text { value }\end{array}$ & Error (\%) \\
\hline Trail 1 & 1.1072 & 1.0052 & -0.9071 & -0.9738 & 1.0327 & 3.2700 \\
\hline Trail 2 & 1.0764 & 0.9977 & -0.6883 & -0.8921 & 1.1387 & 13.8700 \\
\hline Trail 3 & 1.0246 & 0.9841 & -0.8870 & -0.9478 & 1.0221 & 2.2100 \\
\hline Trail 4 & 0.9245 & 1.0302 & -1.0296 & -0.9989 & 1.0280 & 2.8000 \\
\hline Trail 5 & 0.9951 & 0.9799 & -0.8949 & -0.9394 & 1.0198 & 1.9800 \\
\hline Trail 6 & 1.0509 & 1.0231 & -0.8316 & -0.9106 & 1.0455 & 4.5500 \\
\hline Trail 7 & 0.9807 & 1.0058 & -1.0396 & -1.0193 & 1.0038 & 0.3800 \\
\hline Trail 8 & 0.9998 & 0.9796 & -0.8535 & -0.8864 & 1.0507 & 5.0700 \\
\hline Trail 9 & 0.9755 & 1.0065 & -1.0176 & -0.9904 & 1.0039 & 0.3900 \\
\hline Trail 10 & 0.9630 & 0.9885 & -0.9836 & -0.9546 & 1.0114 & 1.1400 \\
\hline Average & 1.0098 & 1.0001 & -0.9133 & -0.9513 & 1.0357 & 3.5700 \\
\hline
\end{tabular}

Table 5.3.2: Solution from the ASA algorithm for a cost function of four independent variables $w$, $x, y$, and $z$

\begin{tabular}{|c|c|c|c|c|c|c|}
\hline \multicolumn{7}{|c|}{ Random Restart Hill Climbing algorithm } \\
\hline & $\mathbf{w}$ & $\mathbf{x}$ & $\mathbf{y}$ & $\mathbf{z}$ & $\begin{array}{c}\text { minimum function } \\
\text { value }\end{array}$ & Error (\%) \\
\hline Trail 1 & -1.0000 & -1.0000 & 1.0000 & 1.0000 & 1.0000 & 0.0000 \\
\hline Trail 2 & -1.0000 & -1.0000 & 1.0000 & 1.0000 & 1.0000 & 0.0000 \\
\hline Trail 3 & 1.0000 & 1.0000 & 1.0000 & 1.0000 & 1.0000 & 0.0000 \\
\hline Trail 4 & 1.0000 & 1.0000 & -1.0000 & -1.0000 & 1.0000 & 0.0000 \\
\hline Trail 5 & 1.0000 & 1.0000 & 1.0000 & 1.0000 & 1.0000 & 0.0000 \\
\hline Trail 6 & 1.0000 & 1.0000 & -1.0000 & -1.0000 & 1.0000 & 0.0000 \\
\hline Trail 7 & -1.0000 & -1.0000 & 1.0000 & 1.0000 & 1.0000 & 0.0000 \\
\hline Trail 8 & 1.0000 & 1.0000 & 1.0000 & 1.0000 & 1.0000 & 0.0000 \\
\hline Trail 9 & 1.0000 & 1.0000 & -1.0000 & -1.0000 & 1.0000 & 0.0000 \\
\hline Trail 10 & 1.0000 & 1.0000 & 1.0000 & 1.0000 & 1.0000 & 0.0000 \\
\hline Average & 0.4000 & 0.4000 & 0.4000 & 0.4000 & 1.0000 & 0.0000 \\
\hline
\end{tabular}

Table 5.3.3: Solution from the RRHC algorithm for a cost function of four independent variables $w, x, y$, and $z$ 
Figure 5.3.1 shows a plot of computation time of all the algorithms for 10 trials each.

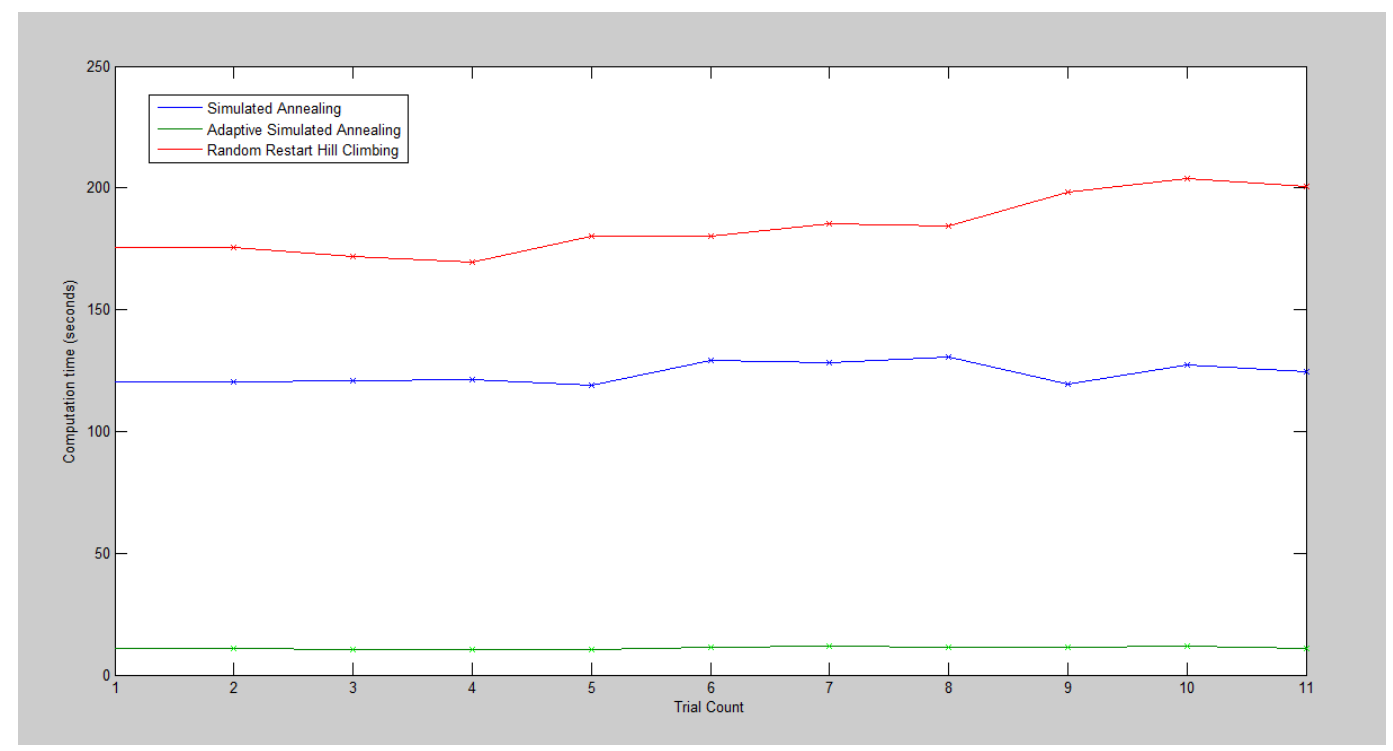

Figure 5.3.1: Computation time (sec.) for 10 tries of the three algorithms

Table 5.3.4 aims to provide information on the tradeoff between percentage error (or accuracy) and computation time in each of the three algorithms

\begin{tabular}{|c|c|c|c|c|c|c|}
\hline & \multicolumn{2}{|c|}{ Simulated Annealing } & \multicolumn{2}{c|}{$\begin{array}{c}\text { Adaptive Simulated } \\
\text { Annealing }\end{array}$} & \multicolumn{2}{c|}{$\begin{array}{c}\text { Random Restart Hill } \\
\text { Climbing }\end{array}$} \\
\hline & Error (\%) & time(sec.) & Error (\%) & time(sec.) & Error (\%) & time(sec.) \\
\hline Trail 1 & 0.0000 & 120.2330 & 3.2700 & 10.8771 & 0.0000 & 175.3429 \\
\hline Trail 2 & 0.0000 & 120.9094 & 13.8700 & 10.5780 & 0.0000 & 171.8625 \\
\hline Trail 3 & 0.0000 & 121.5211 & 2.2100 & 10.5127 & 0.0000 & 169.7304 \\
\hline Trail 4 & 0.0000 & 119.1562 & 2.8000 & 10.6375 & 0.0000 & 180.2140 \\
\hline Trail 5 & 0.0000 & 129.1668 & 1.9800 & 11.3135 & 0.0000 & 180.2062 \\
\hline Trail 6 & 0.0000 & 128.1730 & 4.5500 & 11.7564 & 0.0000 & 185.0980 \\
\hline Trail 7 & 0.0000 & 130.7918 & 0.3800 & 11.1606 & 0.0000 & 184.5443 \\
\hline Trail 8 & 0.0000 & 119.3853 & 5.0700 & 11.1768 & 0.0000 & 198.1524 \\
\hline Trail 9 & 0.0000 & 127.3835 & 0.3900 & 11.7708 & 0.0000 & 204.0424 \\
\hline Trail 10 & 0.0000 & 124.3711 & 1.1400 & 10.9535 & 0.0000 & 200.5978 \\
\hline Average & 0.0000 & 124.1091 & 3.5660 & 11.0737 & 0.0000 & 184.9791 \\
\hline
\end{tabular}

Table 5.3.4: Computation time (sec.) vs. percentage error of the three algorithms for the given cost function of four independent variables 
Figure 5.3.2 shows a scatter plot of the computation time (sec.) vs. percentage error (hence accuracy) tradeoff in each of the three algorithms. The points with a glow represent the average computation time (sec.) vs. average error (\%) for each algorithm's implementation.

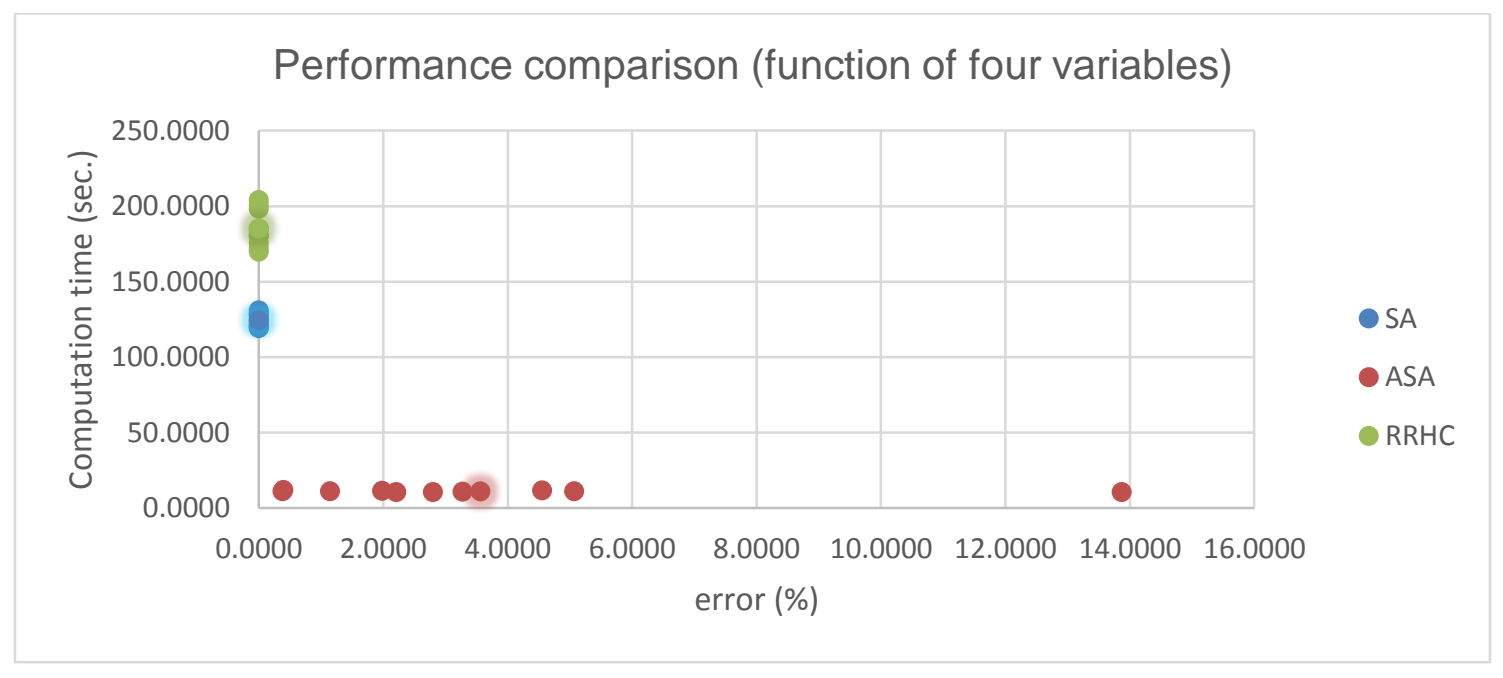

Figure 5.3.2: Computation time (sec.) vs. percentage error (hence accuracy) tradeoff in each of the three algorithms. 


\section{Conclusions}

As is evident from the computation time plots, $\mathrm{RRHC}$ is the computationally most expensive algorithm among the three. The computation times for SA and ASA algorithms are comparable when working with functions of two or three independent variables. However, the difference is significant when working with a function of four independent variables.

$\mathrm{SA}$ and RRHC algorithms deliver higher accuracy in comparison to the ASA algorithm. This is evident from the scatter plots for these three algorithms for each of the tested functions. The tradeoff is that $\mathrm{SA}$ and $\mathrm{RRHC}$ algorithms take more computation time when compared to the ASA algorithm.

Also, it was noted that the RRHC was not able to trace out all the possible optimal solutions in the same number of iterations when implemented on the function of four variables. What this implies is that more iterations may be necessary for RRHC for relatively complex functions.

Given the performance comparison of the three algorithms and their individual accuracy vs. computation time tradeoffs, it is up to the implementer to decide which of the three algorithms would best suit the task at hand. 


\section{Appendix 1}

\section{Main file in MATLAB (for function of two variables):}

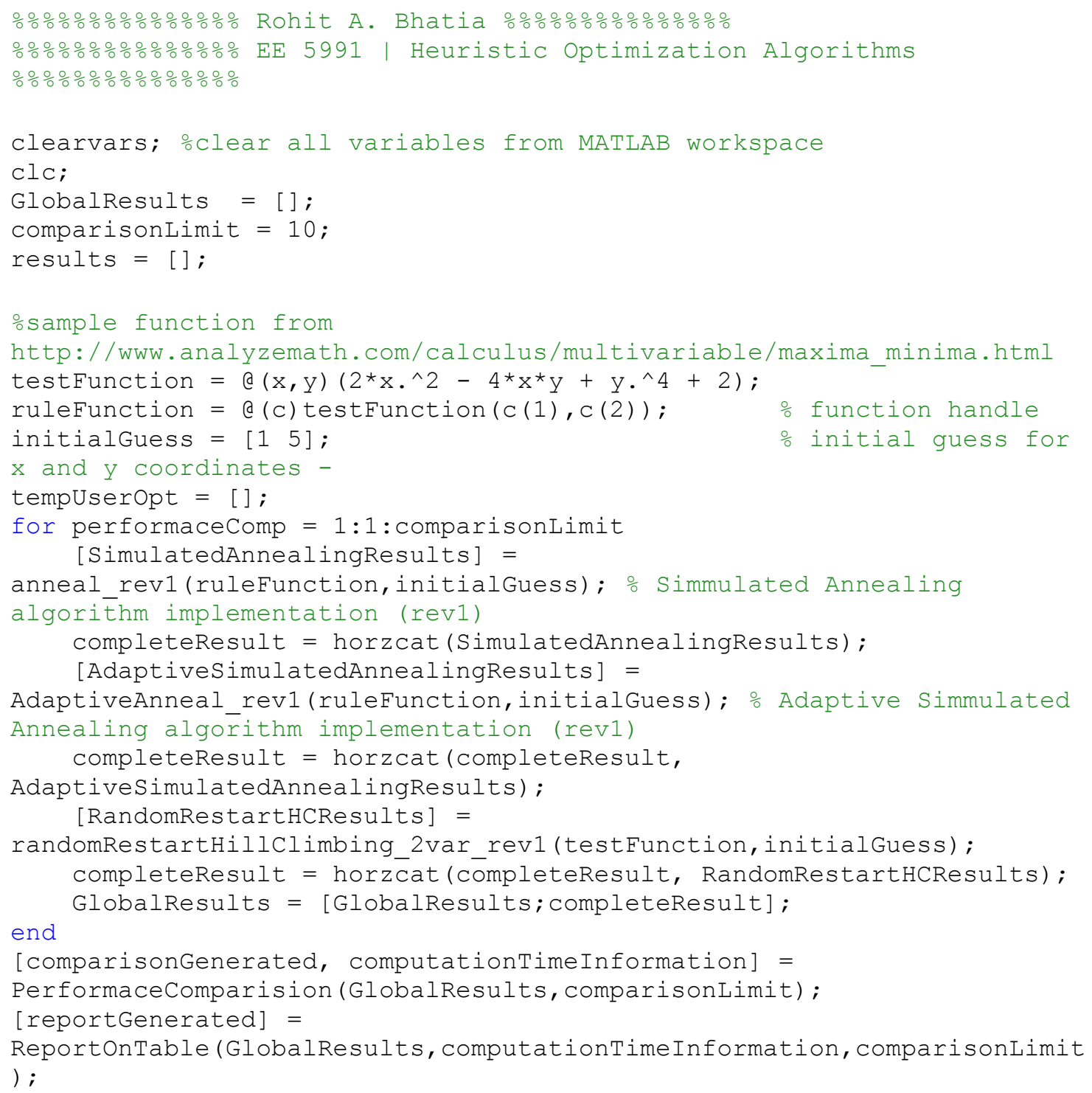

Main file in MATLAB (for function of three variables):

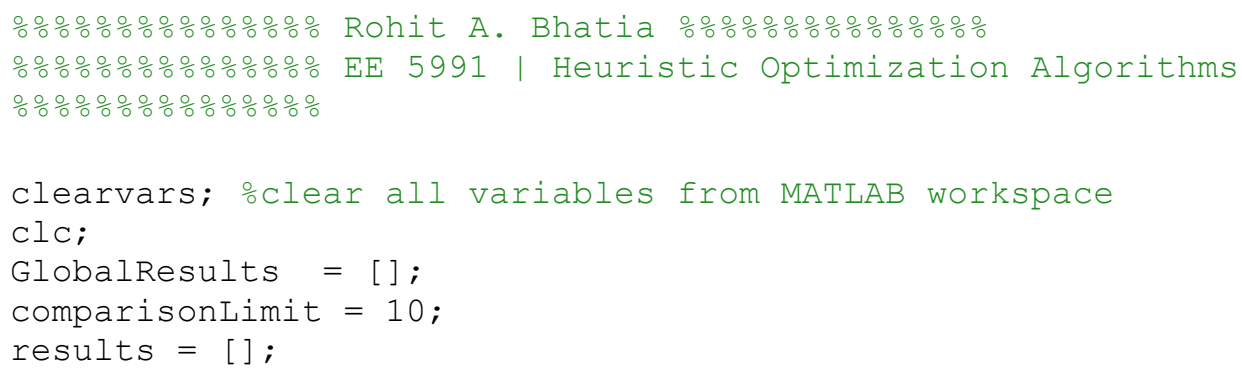




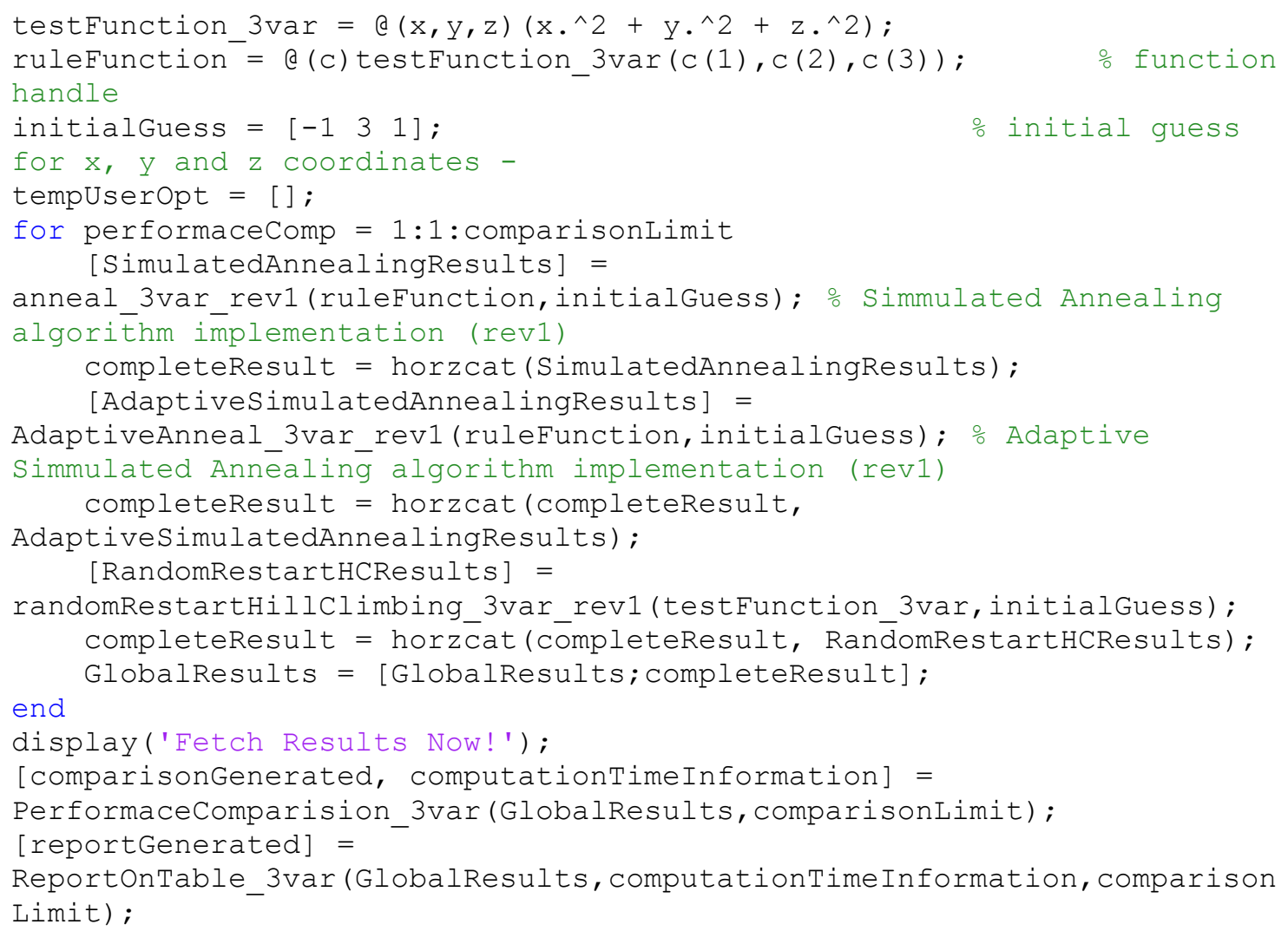

\section{Main file in MATLAB (for function of four variables):}

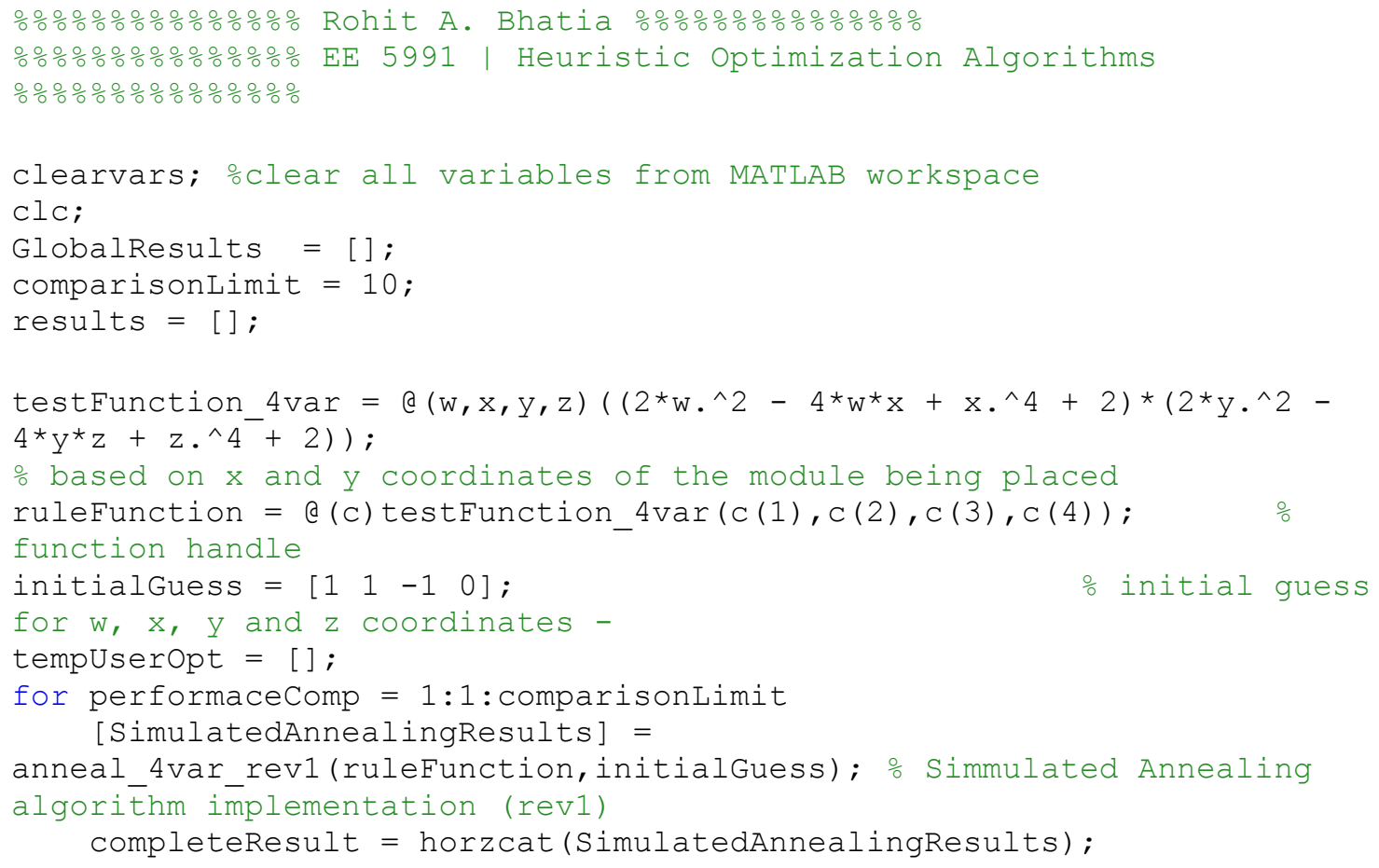


[AdaptiveSimulatedAnnealingResults] =

AdaptiveAnneal 4var revl(ruleFunction, initialGuess); ․ Adaptive

Simmulated Annealing algorithm implementation (rev1)

completeResult = horzcat (completeResult,

AdaptiveSimulatedAnnealingResults);

[RandomRestartHCResults] =

randomRestartHillClimbing_4var_rev1 (testFunction_4var,initialGuess); completeResult = horzcat (completeResult, RandomRestartHCResults);

end GlobalResults = [GlobalResults; completeResult $]$;

[comparisonGenerated, computationTimeInformation] =

PerformaceComparision 4var(GlobalResults, comparisonLimit) ;

[reportGenerated] $=$

ReportonTable 4var(GlobalResults, computationTimeInformation, comparison

Limit);

\section{Non-adaptive simulated annealing algorithm implementation in MATLAB:}

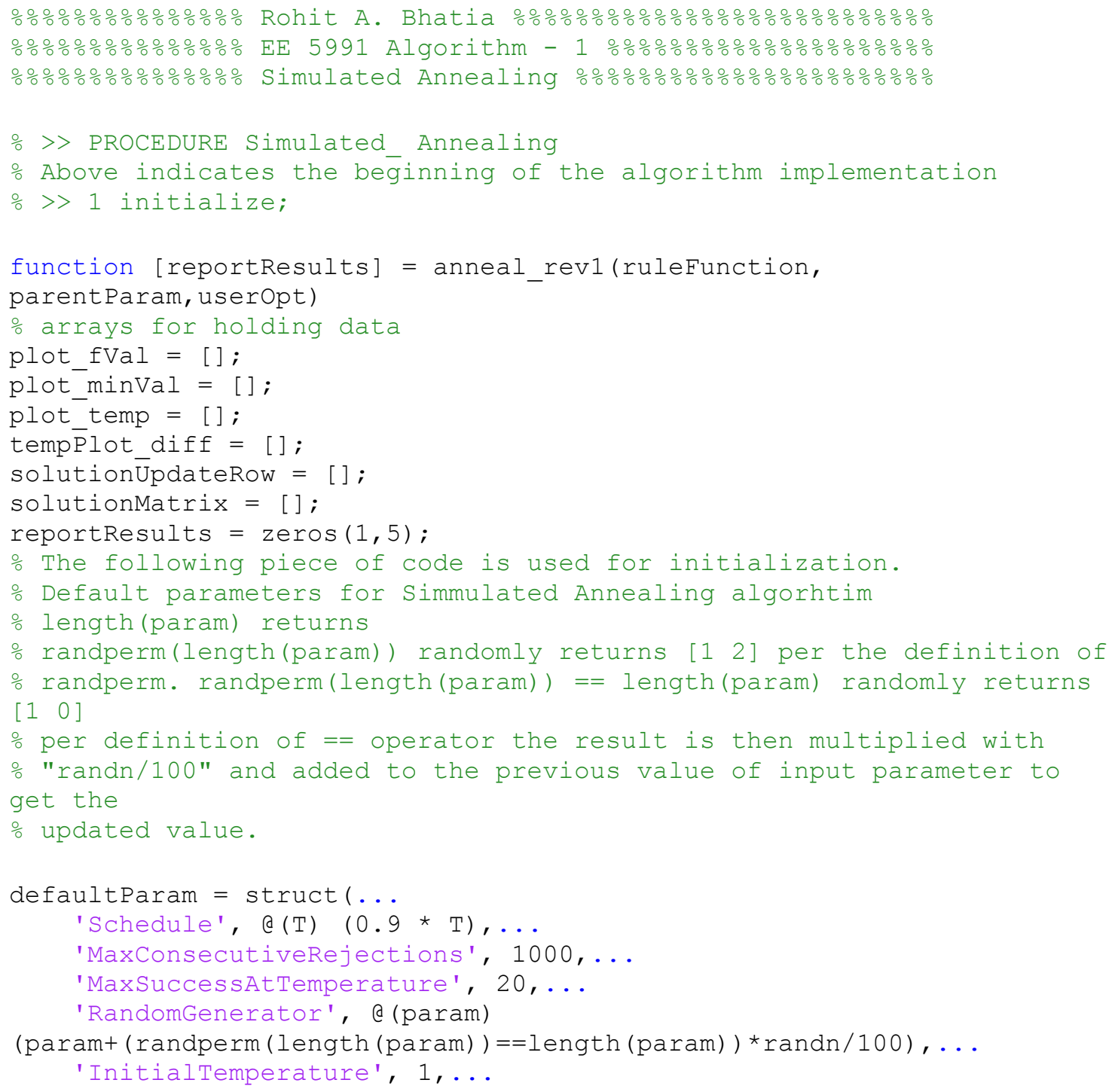




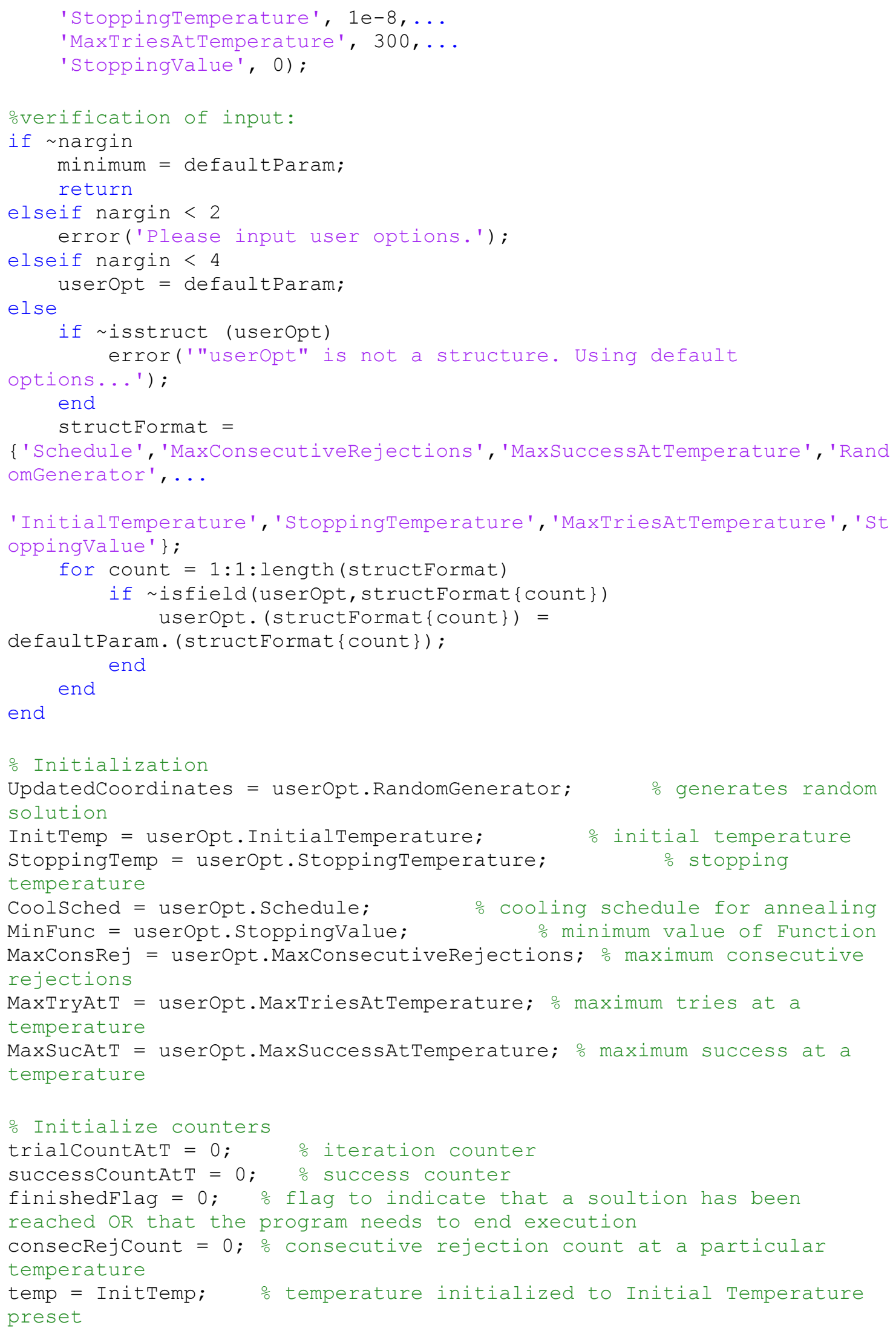


initialLength = ruleFunction(parentParam); \% initial wire length is computed based on the initial guess parameters

oldLength = initialLength; 으릭ize oldLength; will be updated on successive iterations

funcCallcount $=0$; $\frac{\circ}{\circ}$ number of times this function was called

annealcount $=0 ; \%$ number of times annealing was done

$\mathrm{k}=1 ;$; Boltzmann constant

ㅇraw $=0$;

응 Figure control parameter for graph:

figl = figure;

set(fig1, 'name', 'PLOT OF SIMMULATED ANNEALING ALGORITHM (NON-

ADAPTIVE) : ', 'numbertitle', 'off')

Results $=[]$;

hList1 = uicontrol (figl, 'Style','text', 'Position', [600 5400 75]);

응 time / performance calculation

tic;

$\div>3$ WHILE stopping. criterion (loop. count, temperature) = FALSE

응응

while $\sim$ finishedFlag

trialCountAtT $=$ trialCountAtT +1 ;

currentParam = parentParam;

$\circ>4$ WHILE inner.loop.criterion = FALSE

\% We do the annealing if the we have reached the maximum number of tries

\% at a particular temperature OR is the number of successful moves is

o greater than or equal to the preset for maximum success count at a

\% particlar temperature. This is based on the additional condition that

\% the algorithm isn't terminated due to the temperature going below the

o stopping temperature OR the consecutive rejection count doesn't exceed

\% the preset for Maximum Consecutive Rejections

if trialCountAtT $>=$ MaxTryAtT || successCountAtT $>=$ MaxSucAtT

if temp <= StoppingTemp || consecRejCount >= MaxConsRej

finishedFlag $=1$;

funcCallCount $=$ funcCallCount + trialCountAtT; break;

else

\% >> 13 temperature <- schedule(loop count, temperature) ;

ocooling is done

temp $=$ Coolsched (temp);

annealcount = annealCount +1 ;

funcCallCount $=$ funcCallCount + trialCountAtT;

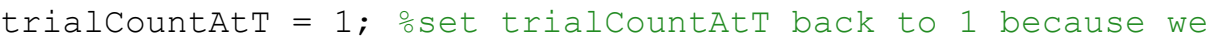
just reduced the temperature

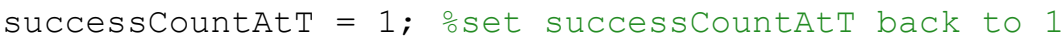

because we just reduced the temperature end

end

\% >> 5 new configuration <- perturb(configuration) ; 
응 This is specific to the wire length implementation. This is where the

\% layout equation will come in and we will optimize it. newParam = UpdatedCoordinates (currentParam); newLength = ruleFunction (newParam);

diff $=$ newLength - oldLength;

\% If new length is less than the minimum value of Length as set then we

\% set the oldLength to the value of the newLength and parentParam as

\% the value obtained for newParam

if (newLength < MinFunc)

parentParam = newParam;

oldLength = newLength;

break

end

o if $f(x 0)-f(x 1)>0$ (in other words the function value decreases,

\% then we replace initial guess with the new guess i.e. parentParam =

\% newParam and oldLength = newLength and count that as a success and

o reset the consecutive rejection count

if (oldLength - newLength > 0)

parentParam = newParam;

oldLength = newLength;

successCountAtT $=$ successCountAtT +1 ;

consecRejCount $=0$;

else

$\%$ if $f(x 0)-f(x 1)<0$ (in other words the function value does

NOT probability

\% decrease AND we still replace $\mathrm{x} 0$ with $\mathrm{xl}$ BUT with the

o given by exp $(-1$ * diff $) / k$ * temp

if (rand $<\exp (-1 *$ diff) $/ \mathrm{k} *$ temp)

parentParam = newParam;

oldLength = newLength;

successCountAtT $=$ successCountAtT +1 ;

\% if the acceptance probability is low, then we reject the

move

else

consecRejCount $=$ consecRejCount +1 ;

end

end

\% Plotting data used only if one iteration is performed for performance

\% comparision

plot_fVal = [plot_fVal;oldLength; ]

plot minVal = [plot minVal; parentParam $]$;

plot_temp = [plot_temp; temp];

temp $\bar{P}$ lot diff $=$ [tempPlot diff;diff];

solutionUpdateRow = [parentParam(1) parentParam(2) oldLength]; 


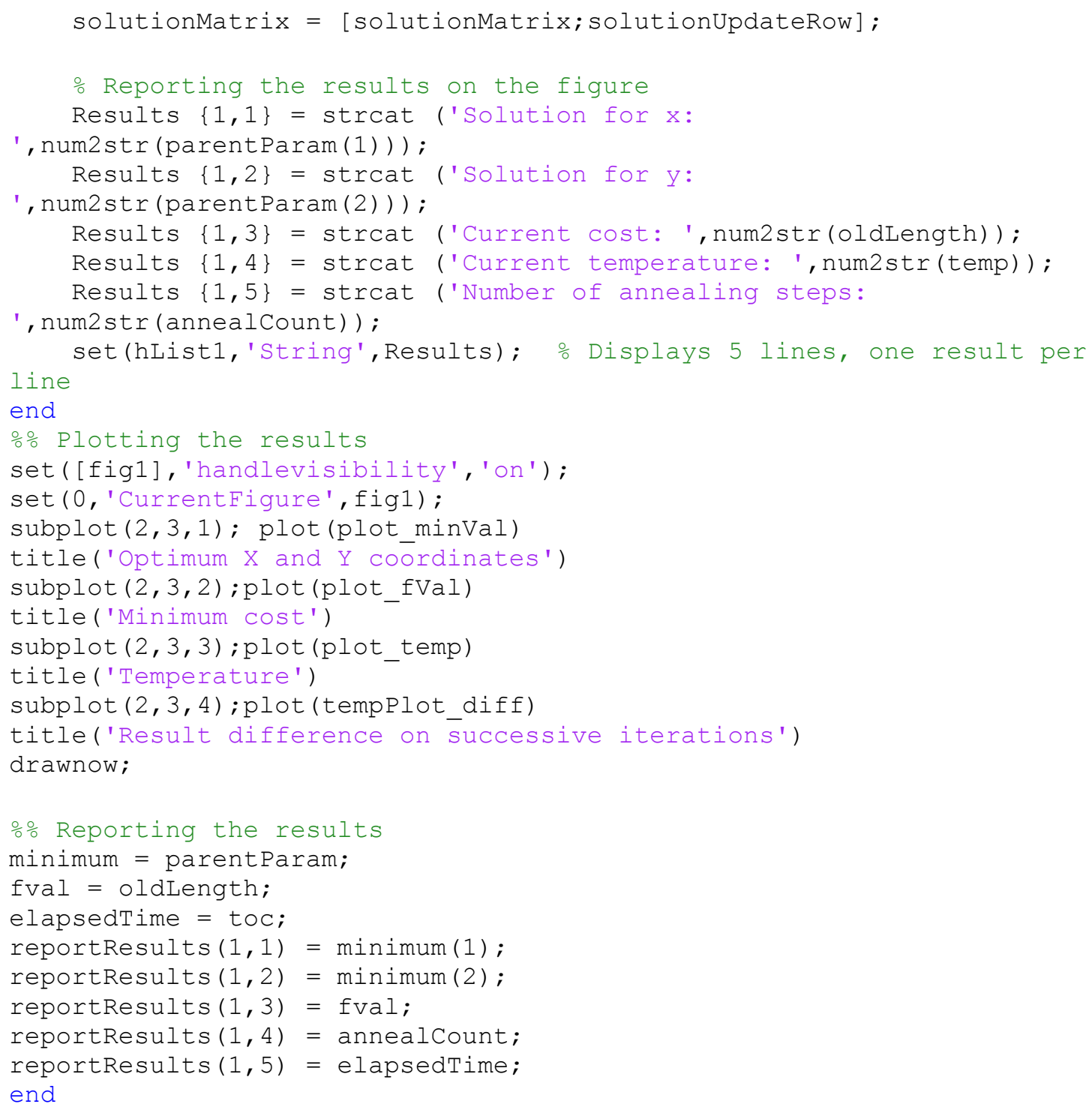

\section{Adaptive simulated annealing algorithm implementation in MATLAB:}

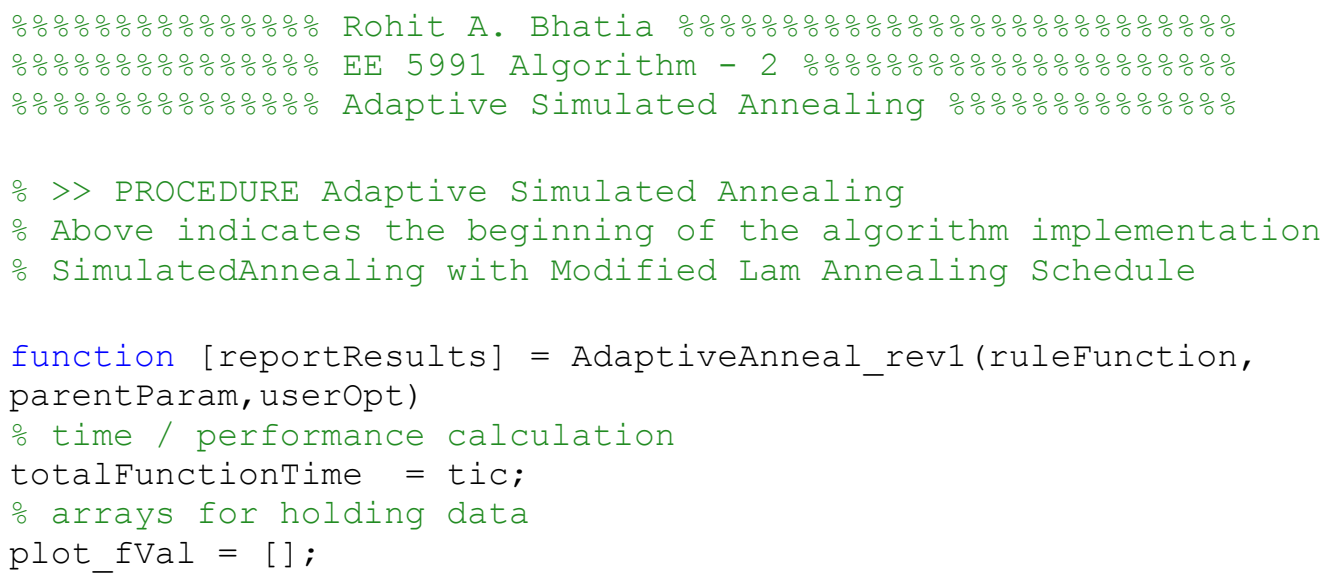




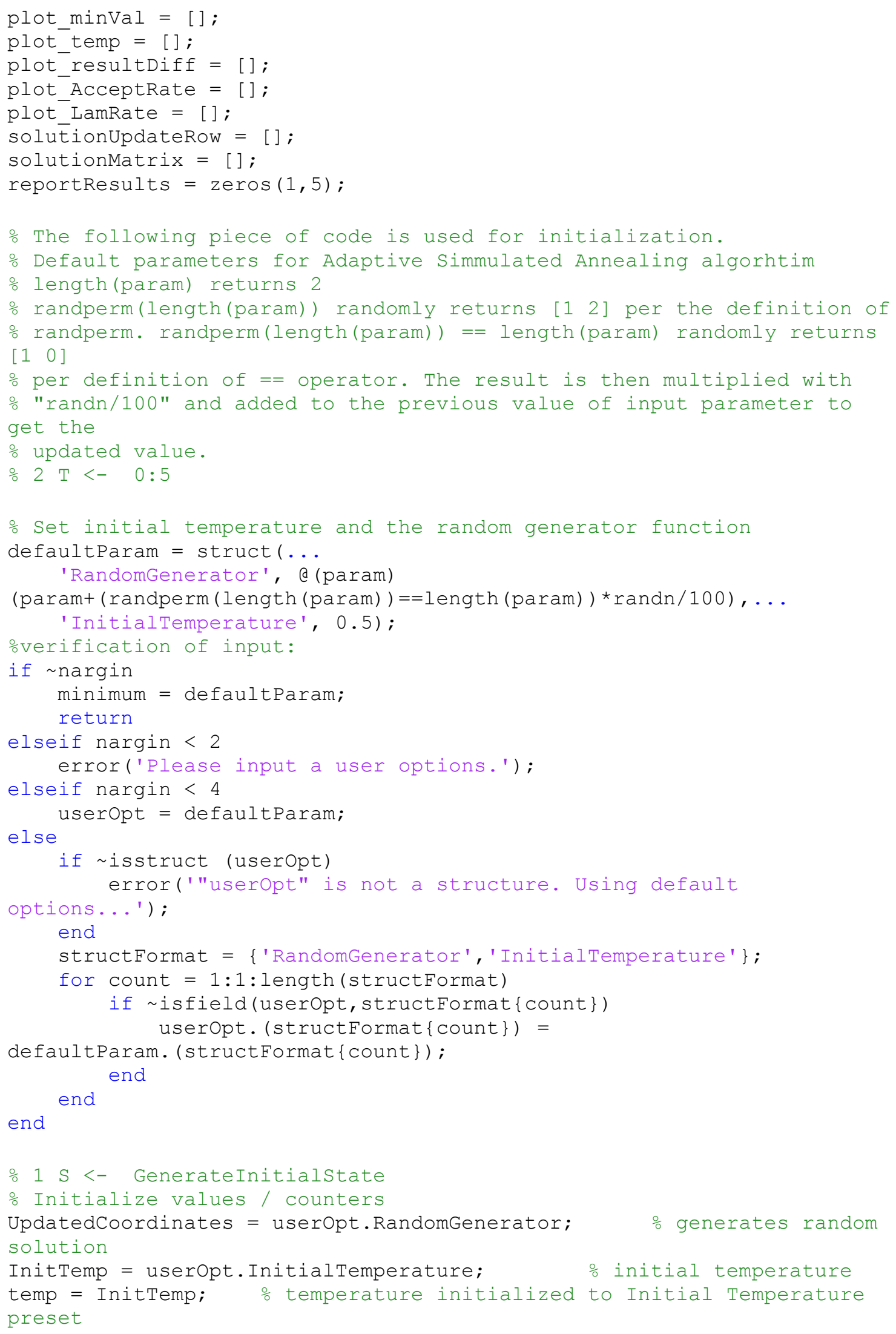




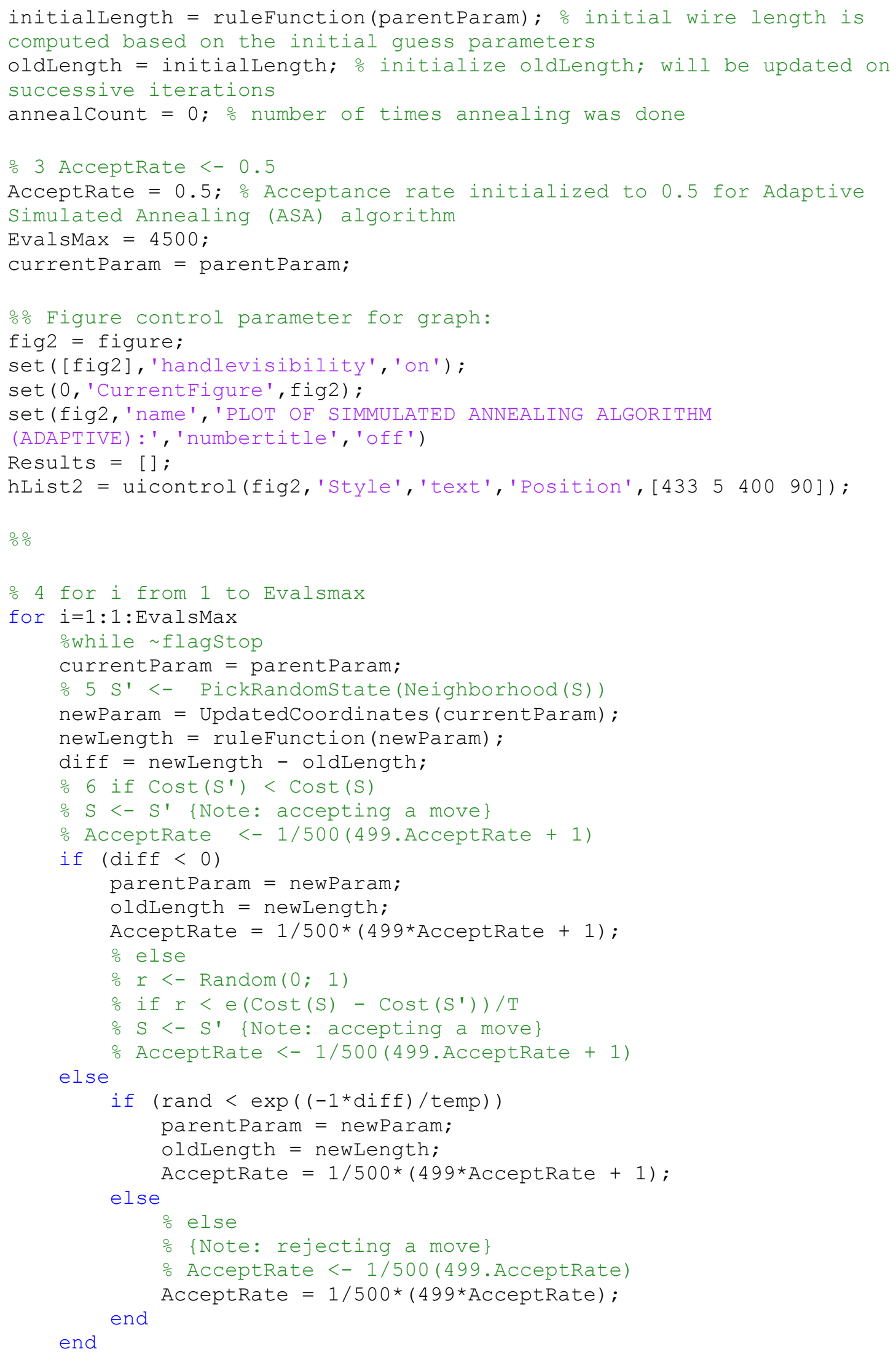




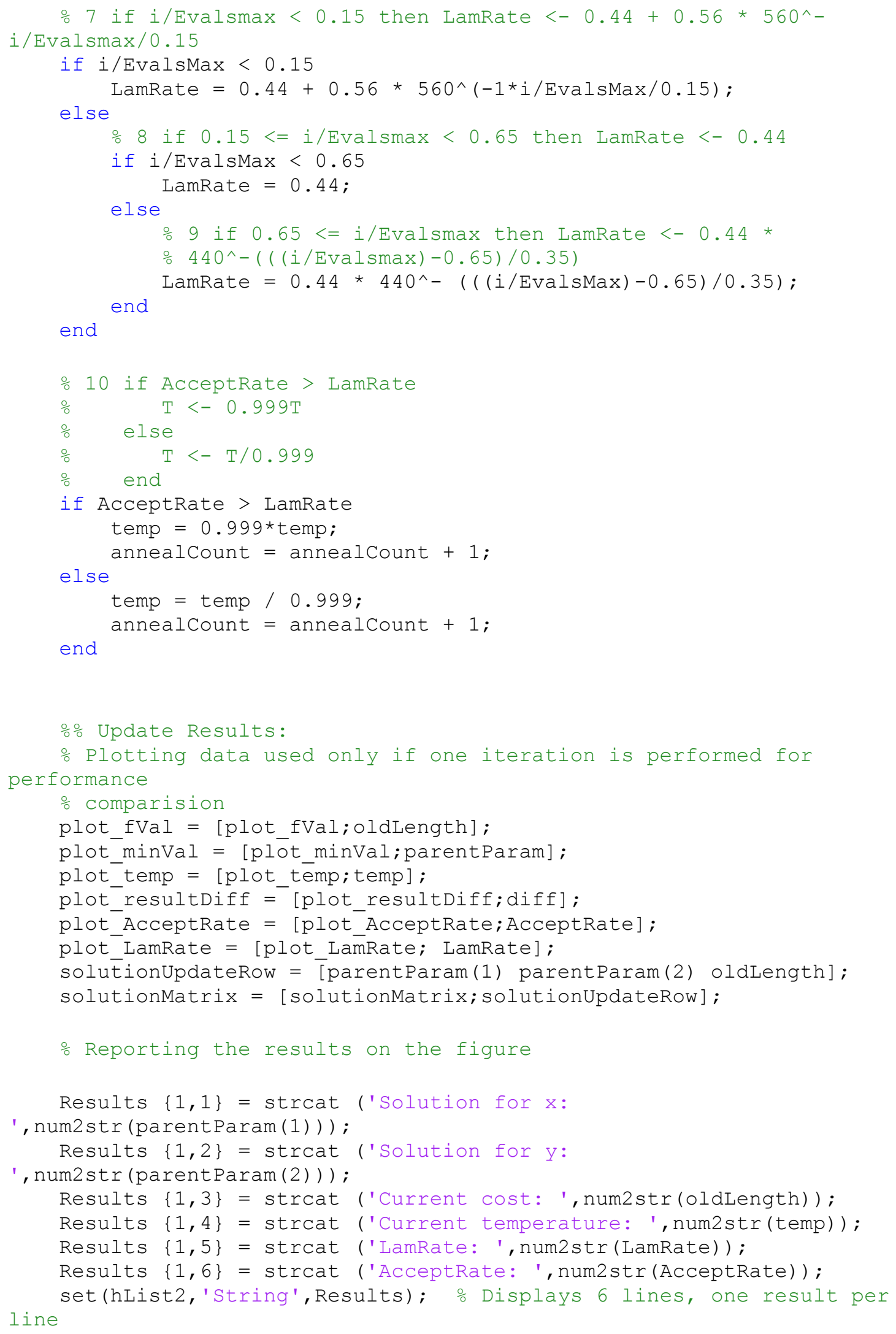




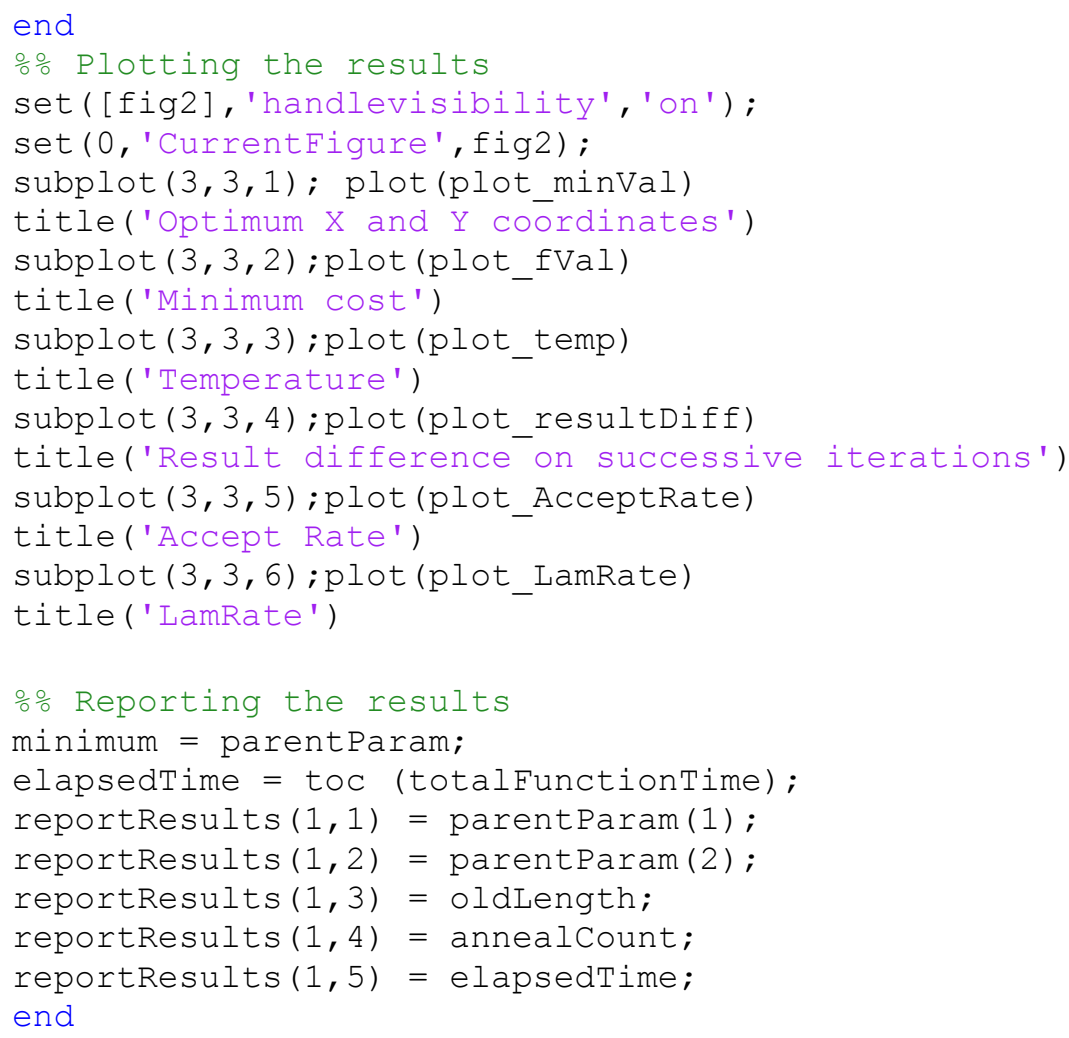

\section{Random Restart Hill Climbing algorithm implementation in MATLAB (for function of two variables):}

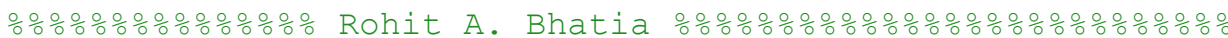

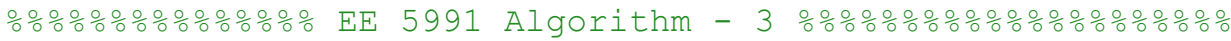




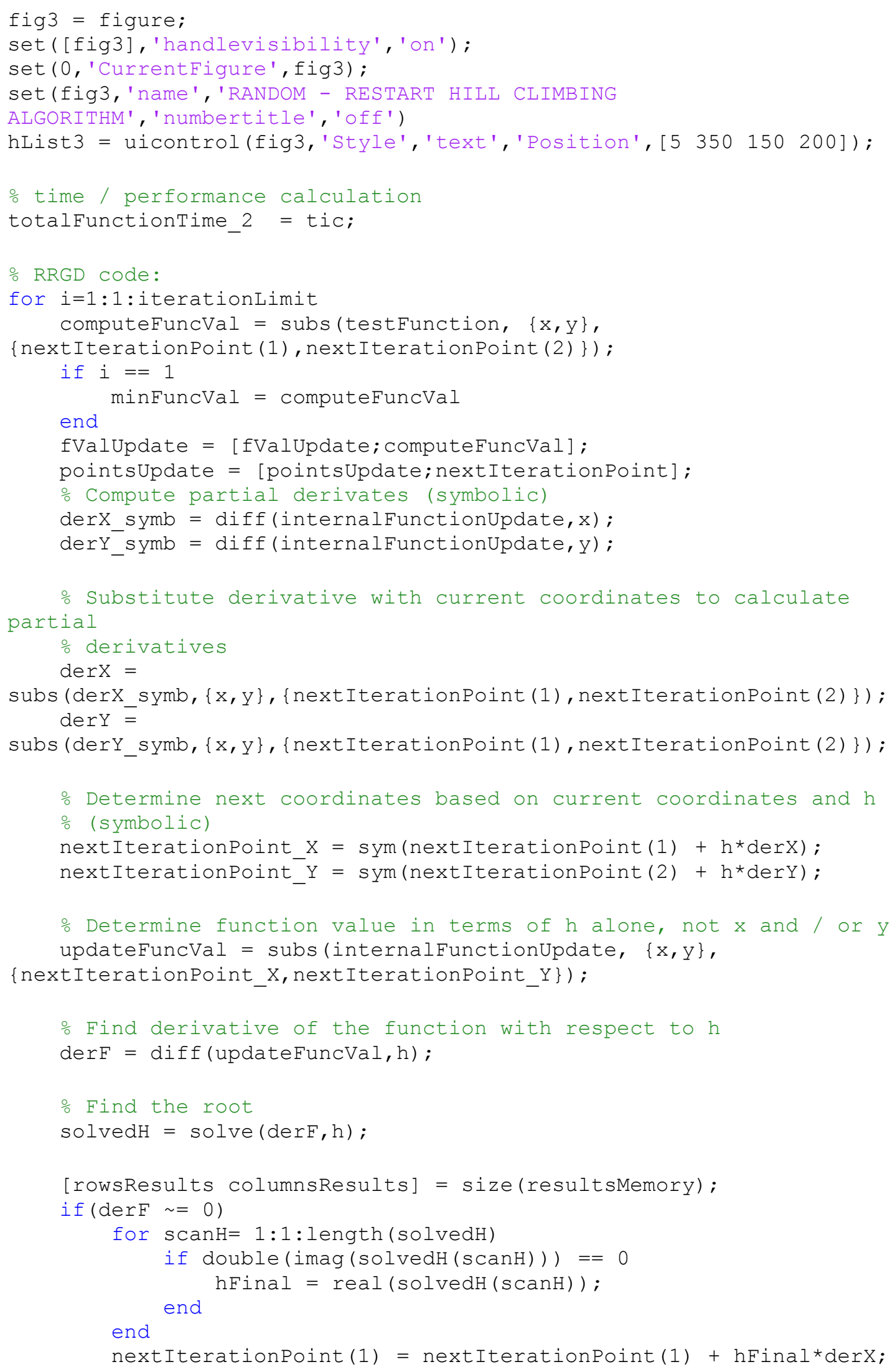




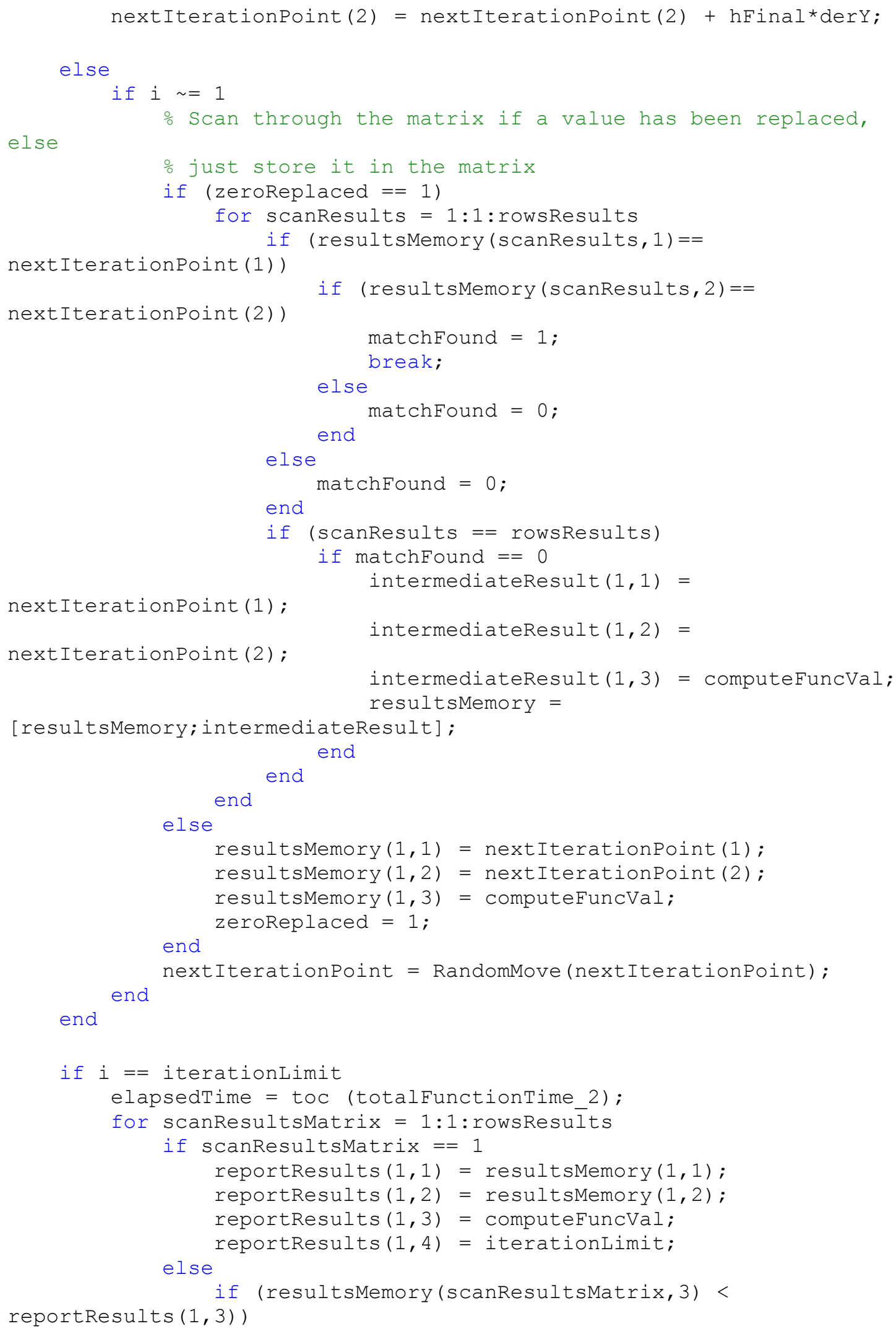




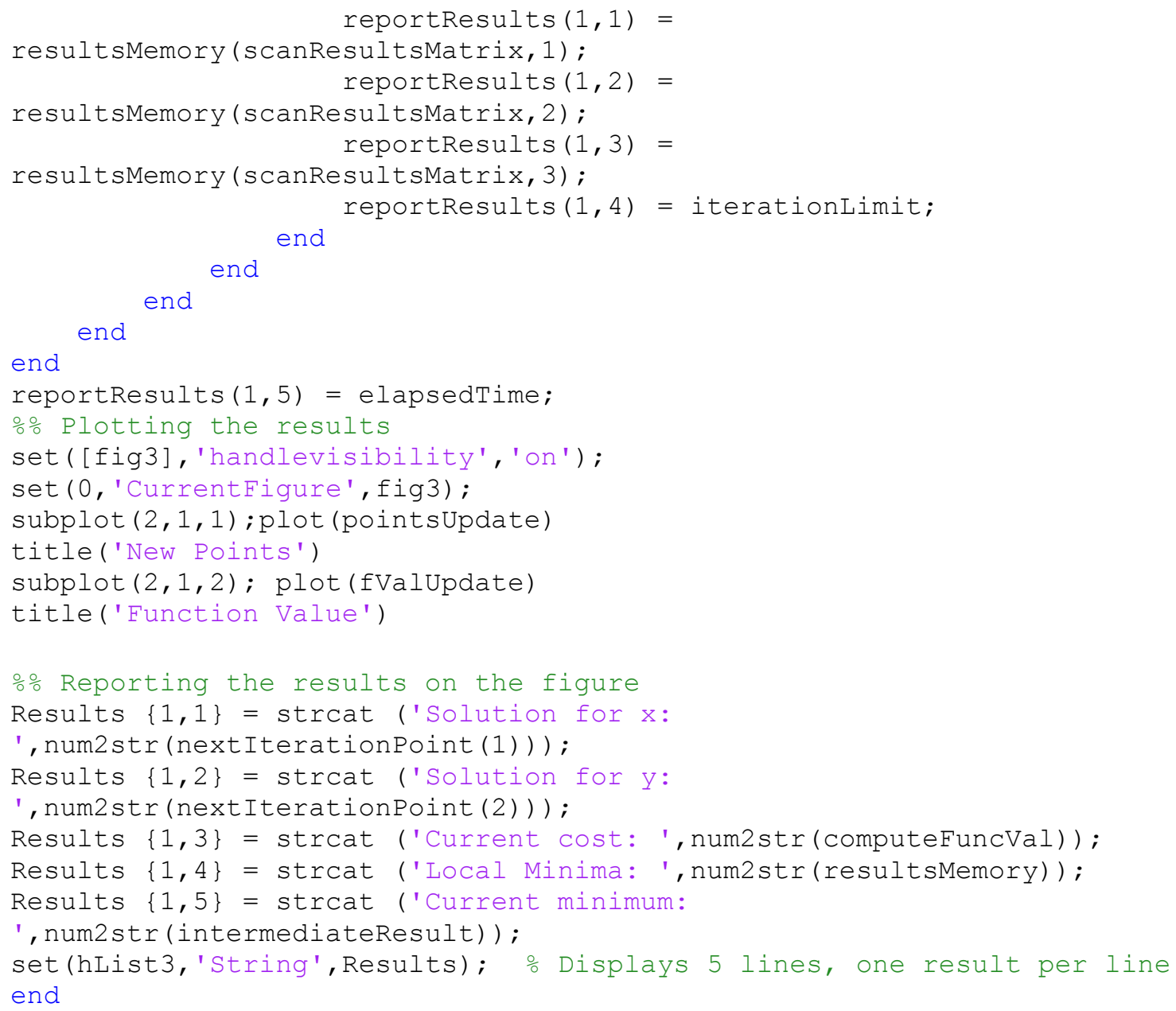

\section{Random Restart Hill Climbing algorithm implementation in MATLAB (for function of three variables):}

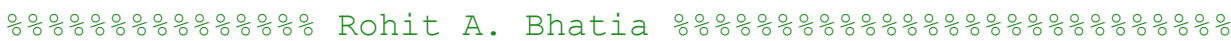

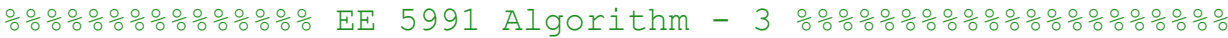




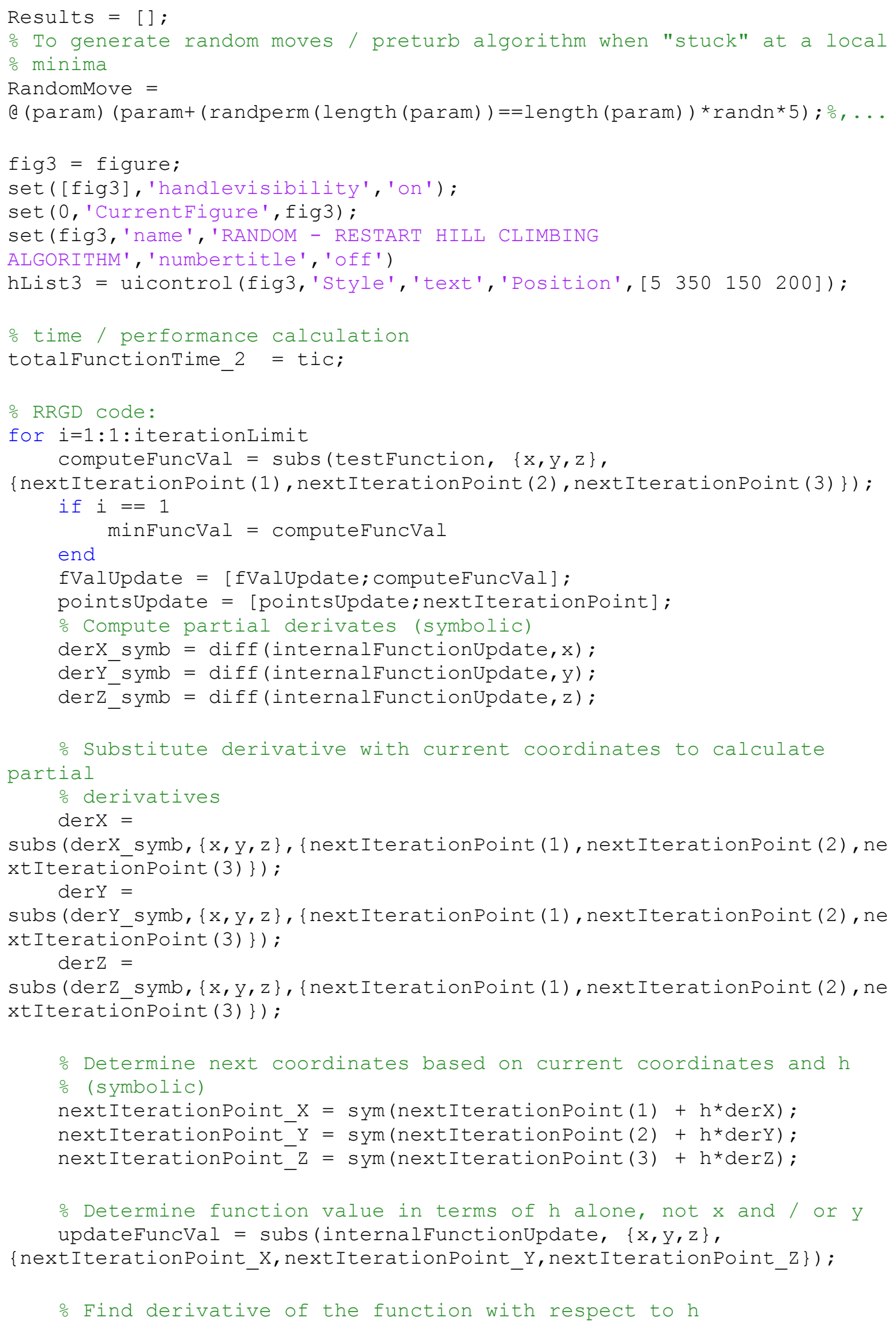




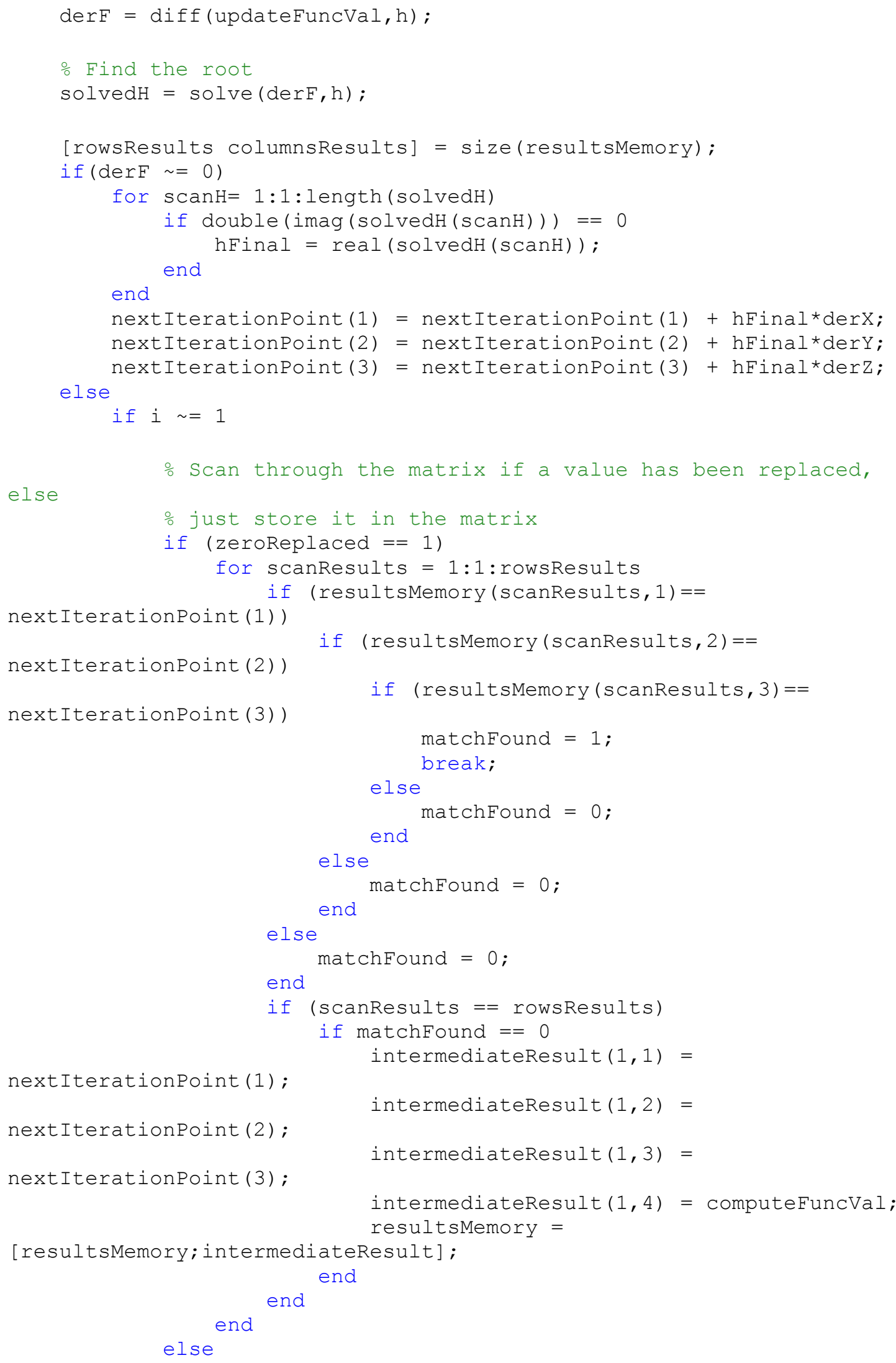




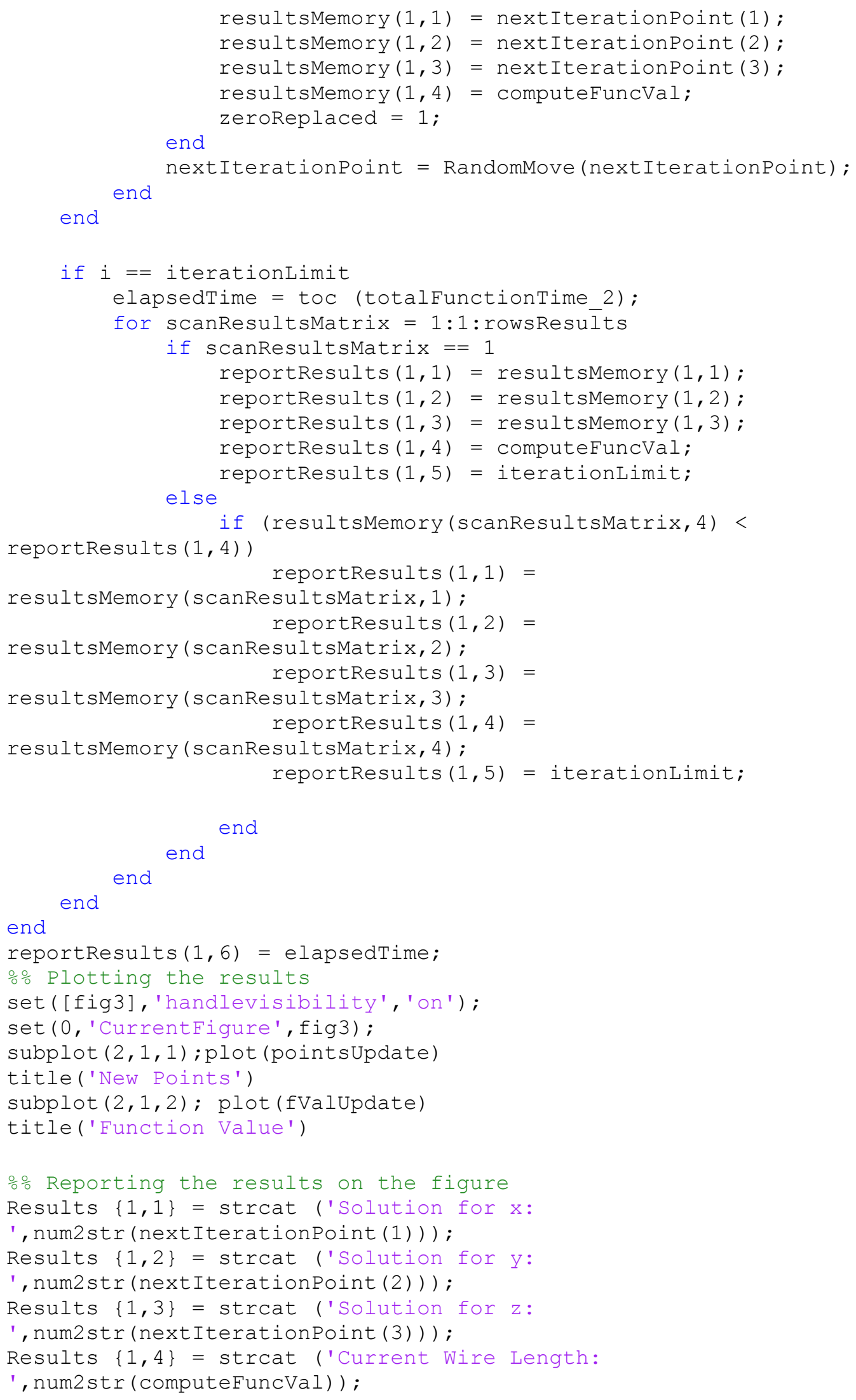




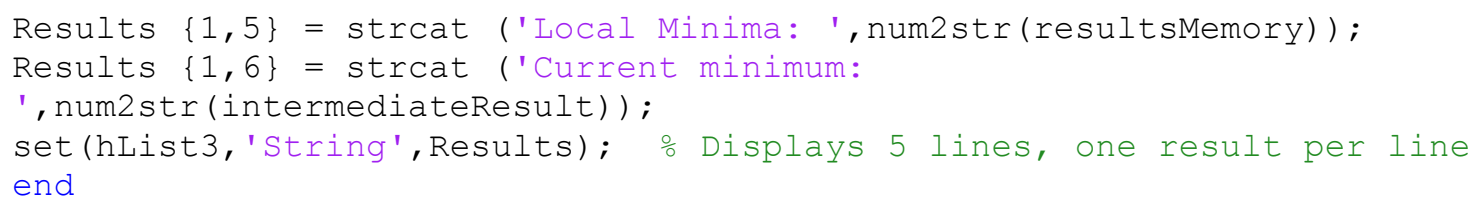

\section{Random Restart Hill Climbing algorithm implementation in MATLAB (for function of four variables):}

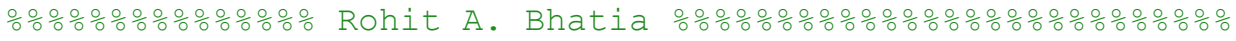

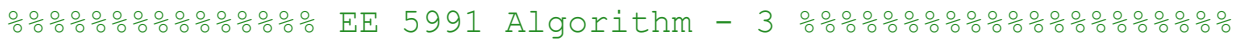

응 $\% \frac{0}{0} \% \circ \%$

function [reportResults] =

randomRestartHillClimbing_4var_rev1 (testFunction, initialGuess)

reportResults $=$ ones $(1,7)$;

syms w x y z a b c d h; osymbols for calculating derivative etc.

nextIterationPoint = initialGuess;

internalFunctionUpdate = testFunction;

fValupdate $=[]$;

pointsupdate $=[]$;

resultsMemory $=\operatorname{zeros}(1,5)$;

intermediateResult $=\operatorname{zeros}(1,5) ;$ For concatenating results to form

the results matrix

zeroReplaced $=0$;

matchFound $=0$;

iterationLimit $=1000$;

Results $=$ [];

\% To generate random moves / preturb algorithm when "stuck" at a local

\% minima

RandomMove $=$

@ (param) $($ param+ (randperm $($ length $($ param $))==$ length $($ param $)) * \operatorname{randn} * 5) ; \circ, \ldots$

fig3 = figure;

set ( [fig3], 'handlevisibility', 'on');

set $(0$, 'CurrentFigure', fig3);

set(fig3, 'name', 'RANDOM - RESTART HILL CLIMBING

ALGORITHM' ', 'numbertitle', 'off' )

hList3 = uicontrol(fig3,' Style','text','Position', [5 350150 200]);

\% time / performance calculation

totalFunctionTime_2 = tic;

\% RRGD code:

for $i=1: 1$ : iterationLimit

computeFuncVal = subs(testFunction, $\{w, x, y, z\}$,

\{nextIterationPoint (1), nextIterationPoint (2), nextIterationPoint (3), nex

tIterationPoint (4) \});

if $i==1$

minfuncVal $=$ computeFuncVal

end

fValupdate $=$ [fValupdate; computeFuncVal $]$;

pointsupdate = [pointsUpdate; nextIterationPoint];

o compute partial derivates (symbolic) 


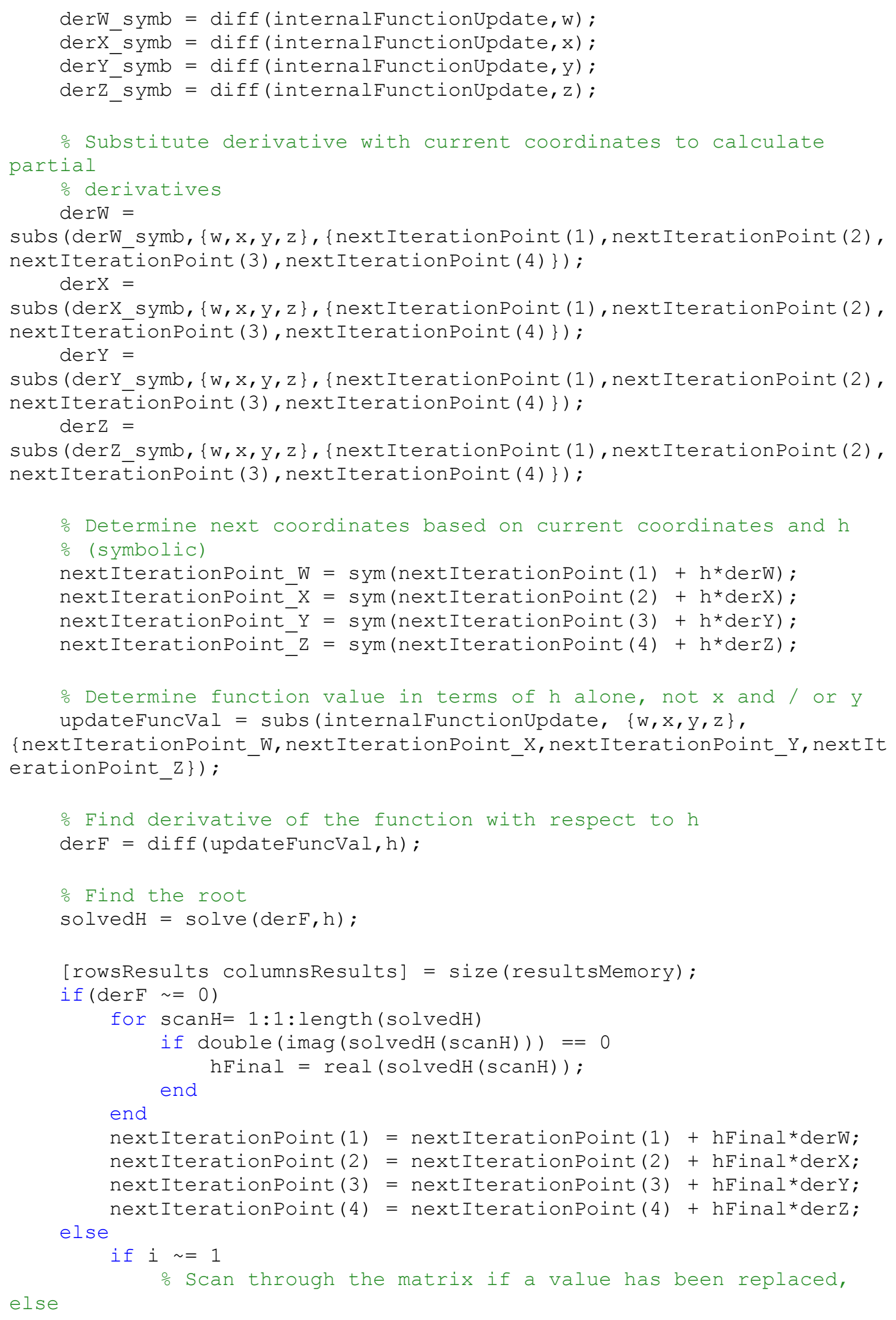




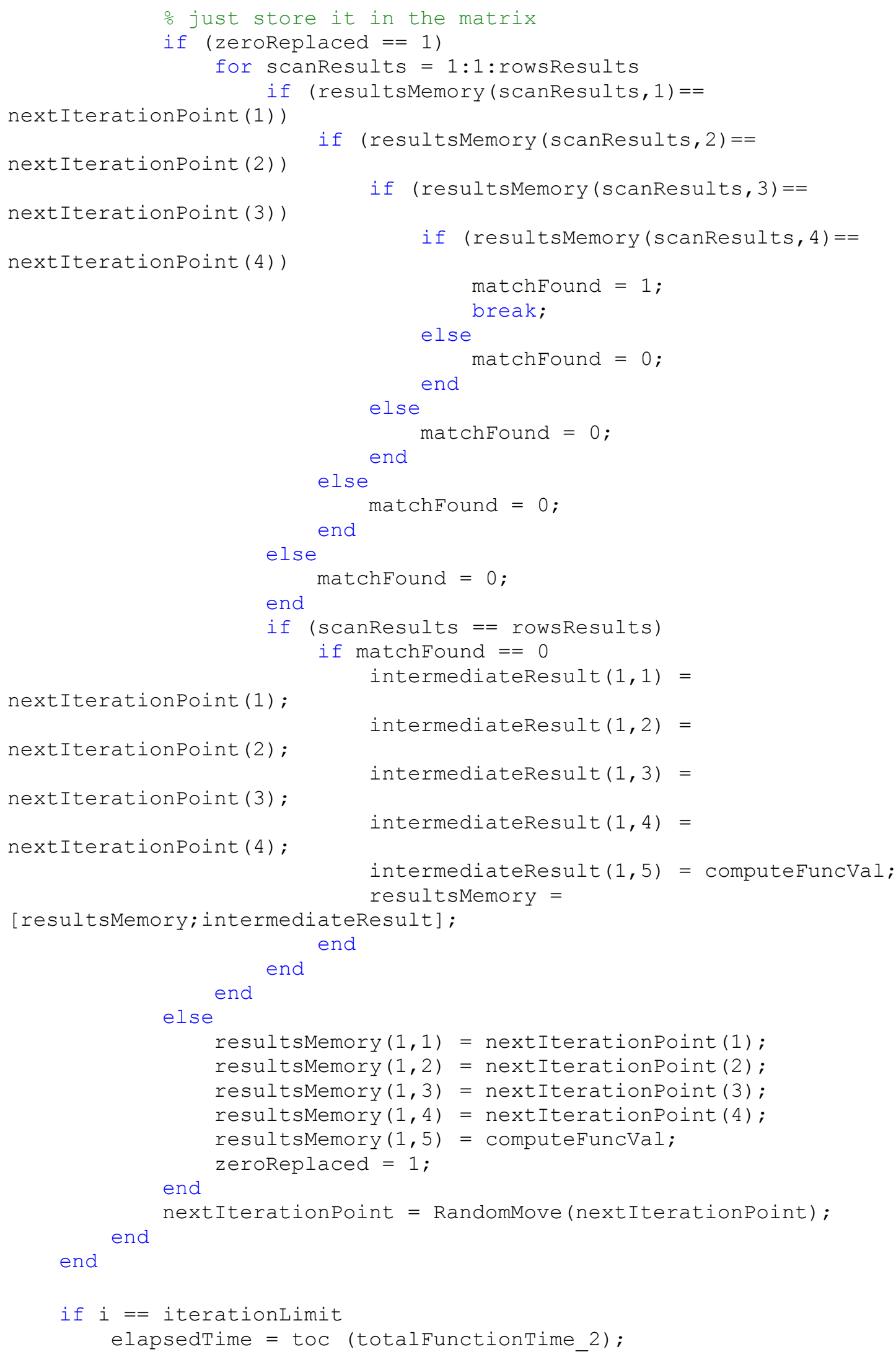




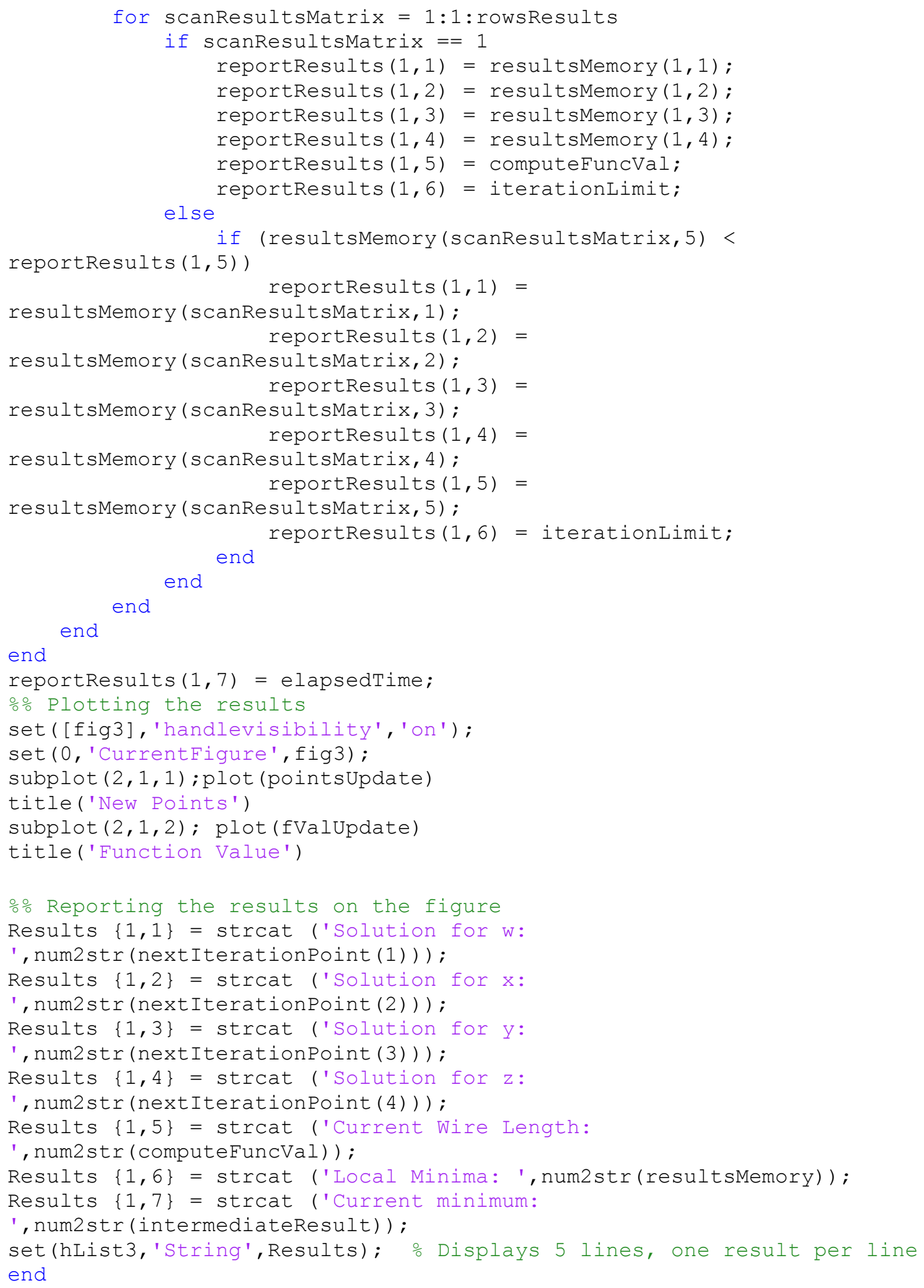




\section{Performance comparison in MATLAB:}

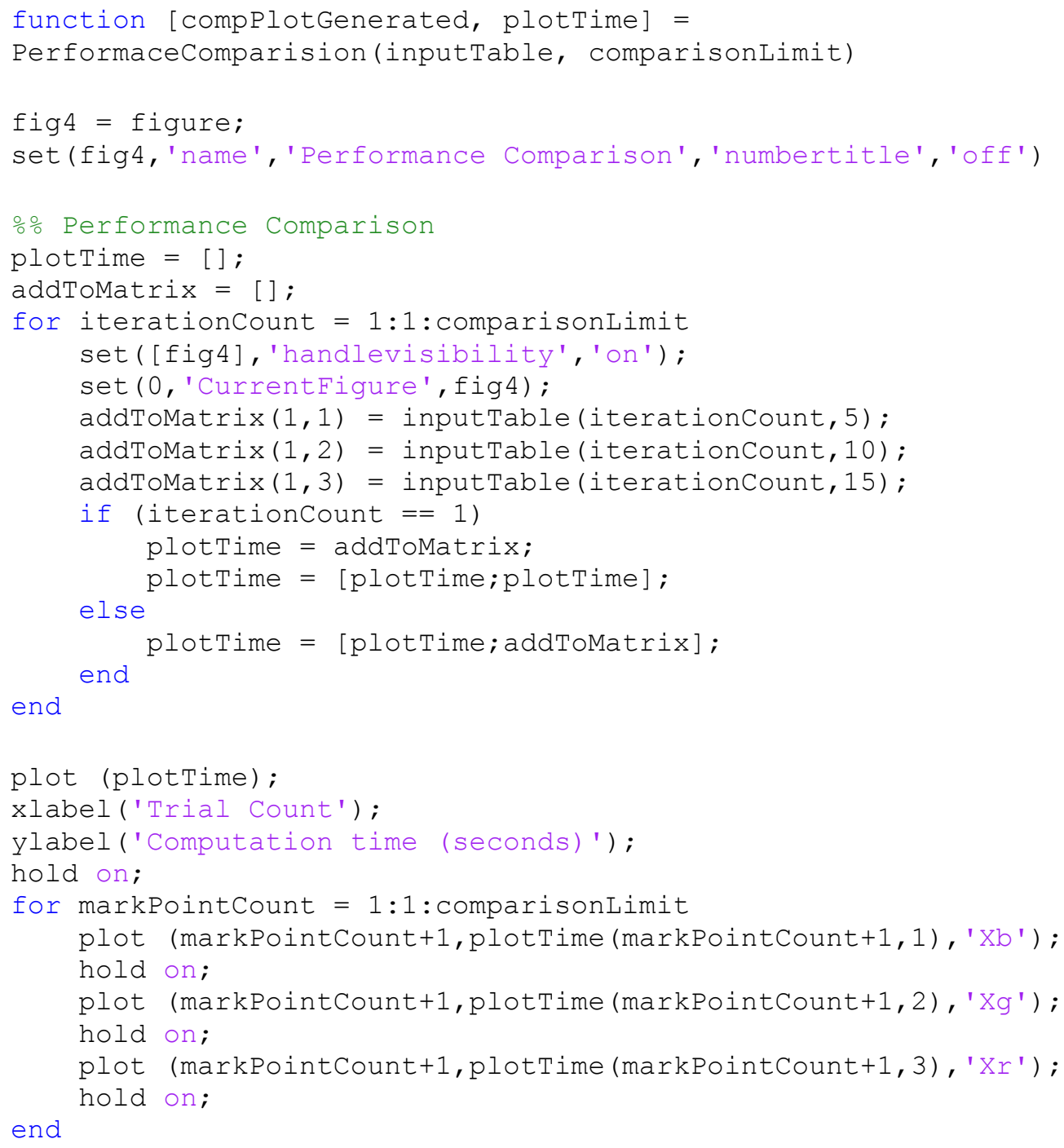

\section{Report generation in MATLAB:}

function [reportGenerated] =

ReportonTable (inputGlobalTable, inputTimeTable, comparisonLimit)

fig 5 figure;

set (fig5, 'name', 'Report', 'numbertitle', 'off', 'Position', [50 2131266 300 ])

\% Framework for reporting results: 


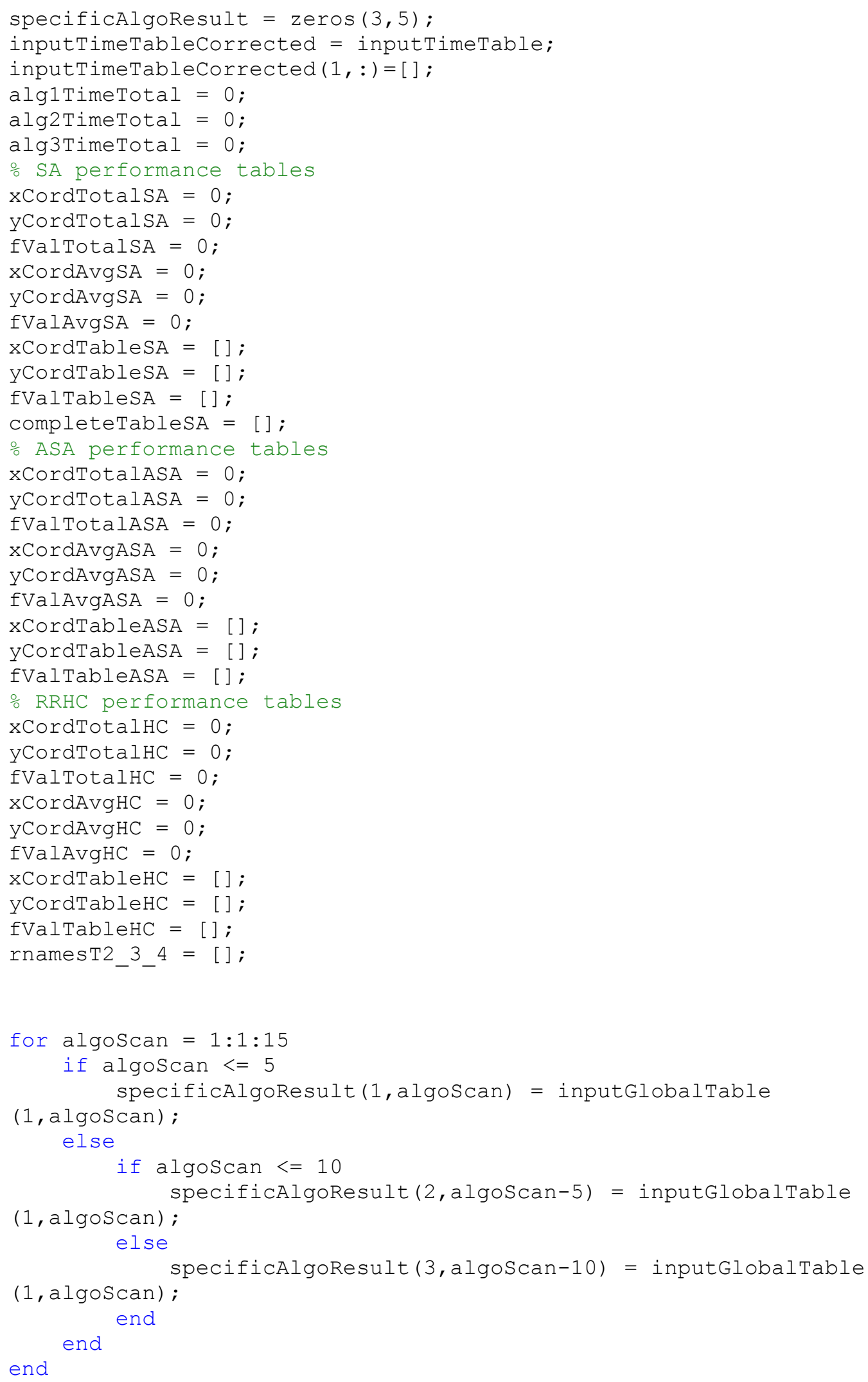




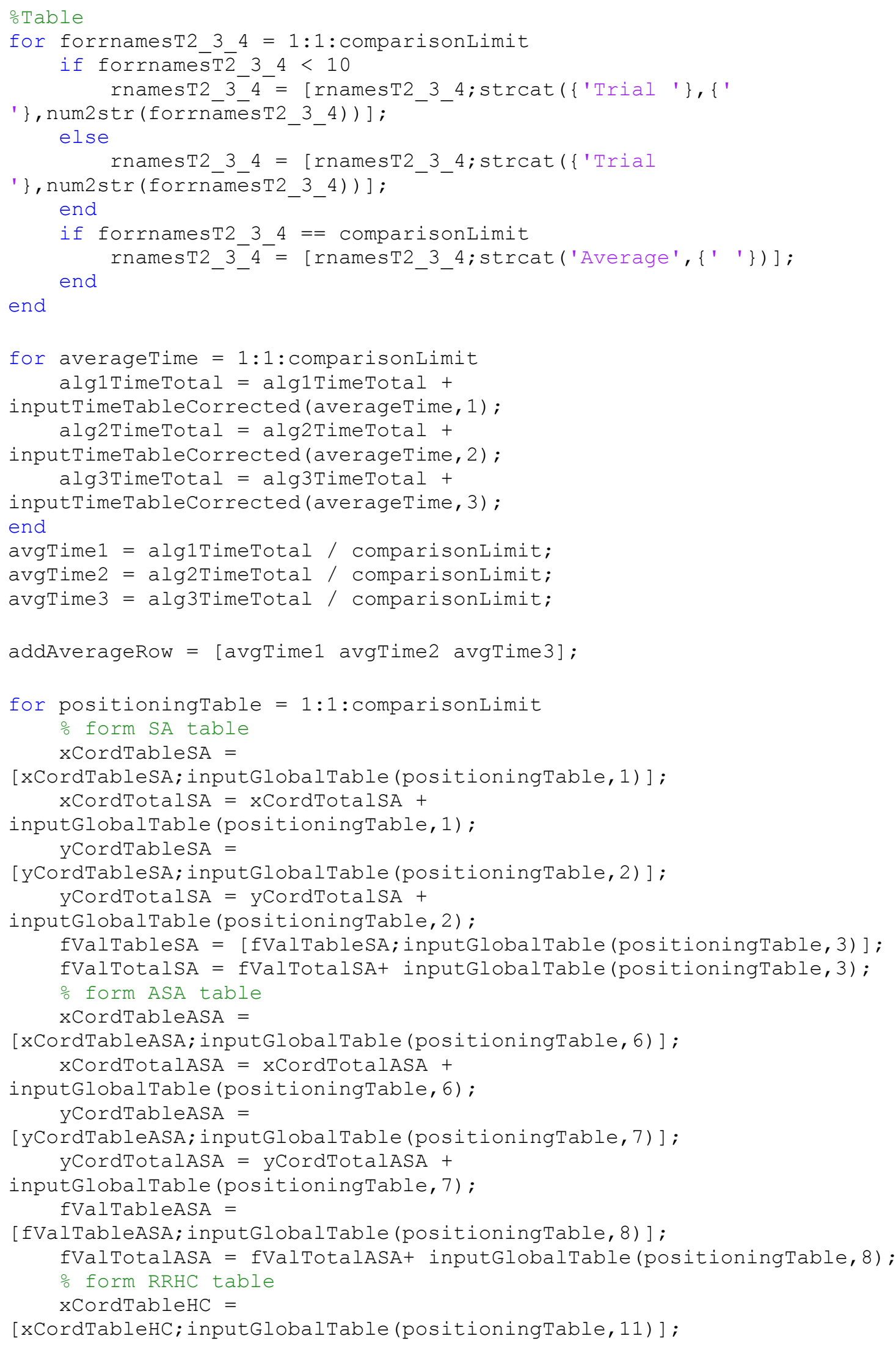




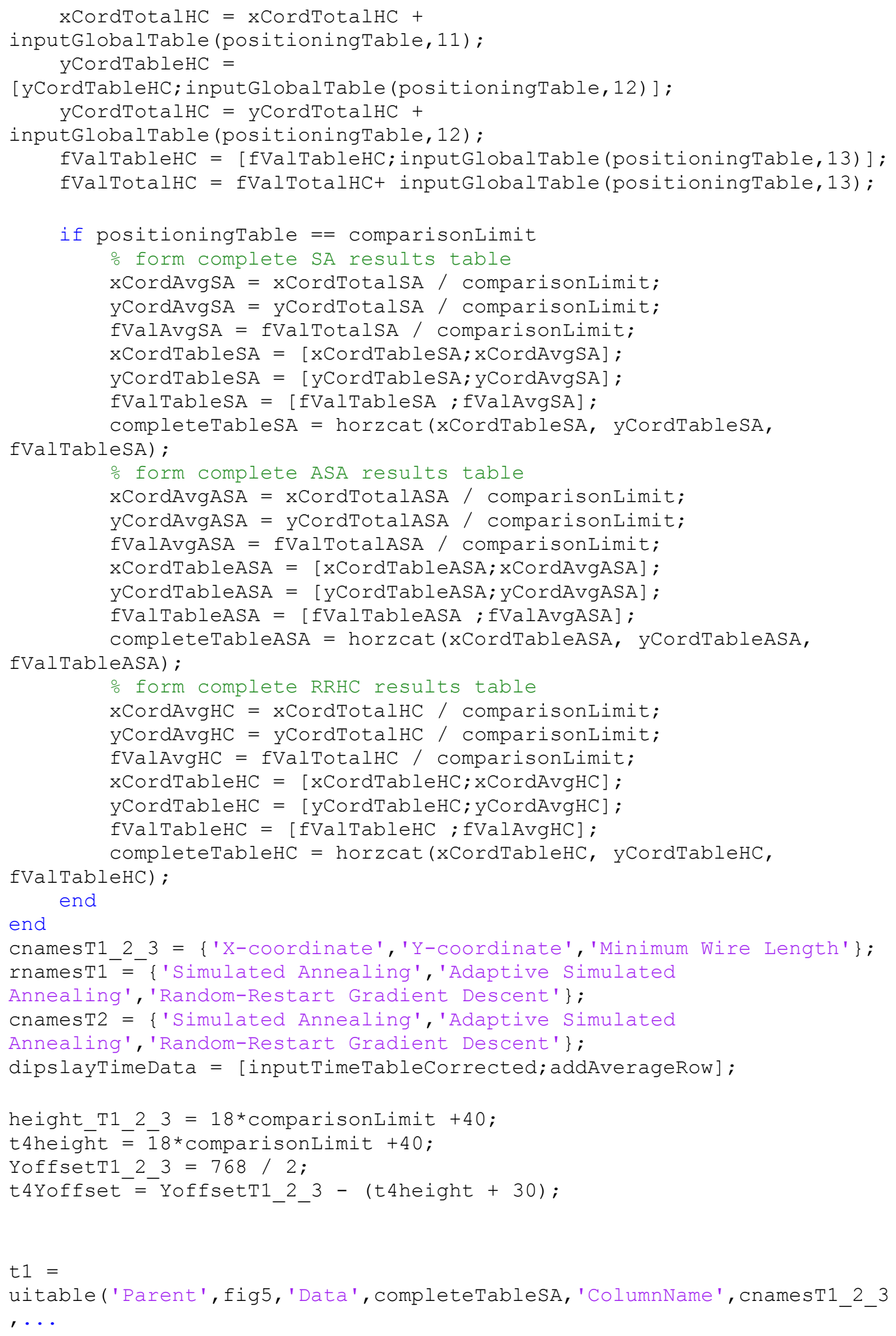




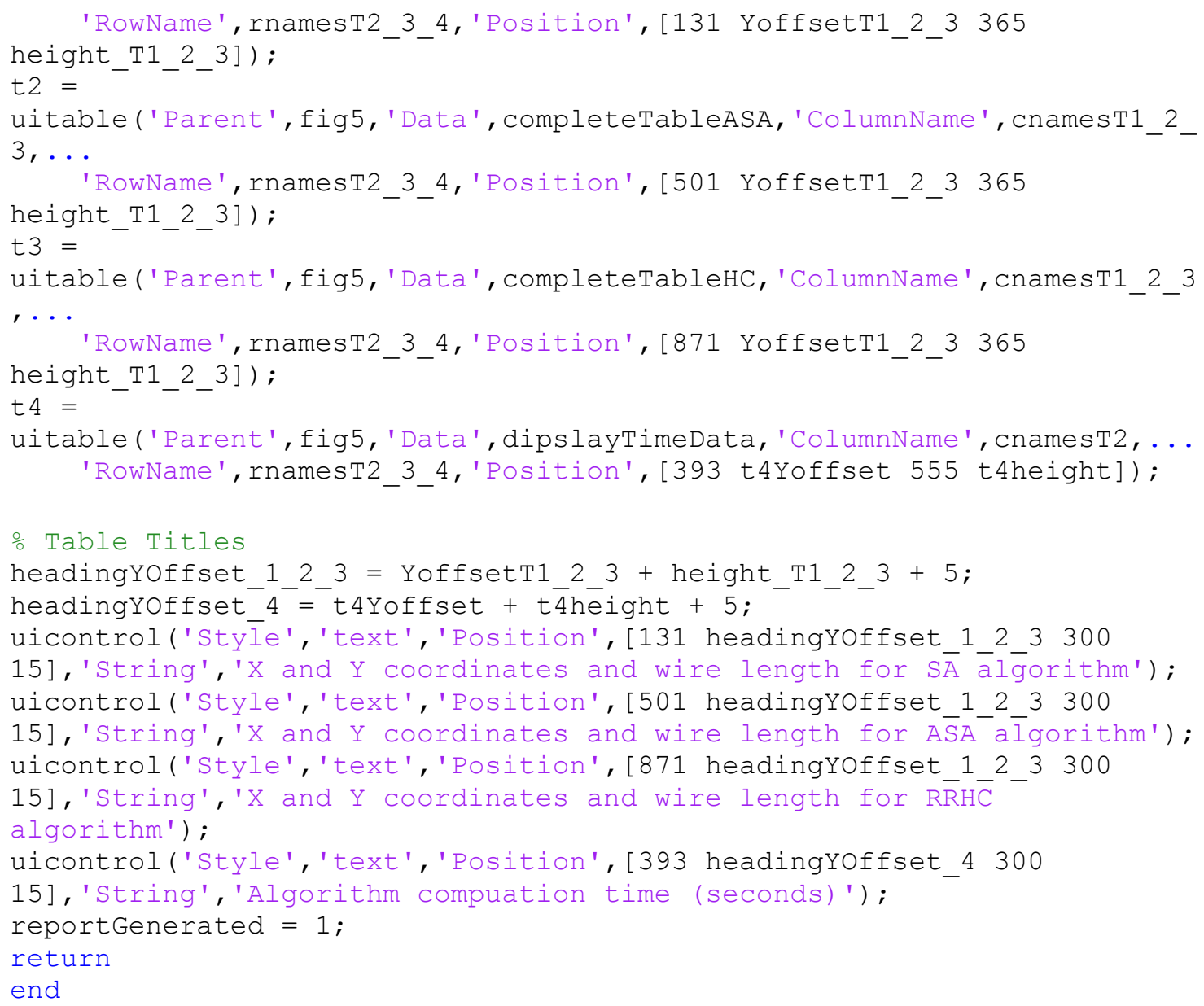




\section{References}

[1] K. Sahookar and P. Mazumder, "VLSI Cell Placement Techniques," ACM Computing Surveys, vol. 23, 1991.

[2] V. .R, P. S. .S, L. .R, and Kumaravel, "Evolutionary Algorithmical Approach for VLSI Physical Design-Placement Problem," ACEEE Int. J. on Electrical and Power Engineering, vol. 2, 2011.

[3] S. M. Sait, M. I. Ali, and A. M. Zaidi, "Multiobjective VLSI Cell Placement Using Distributed Simulated Evolution Algorithm," 2005.

[4] A. Dekkers and E. Aarts, "Global optimization and simulated annealing," Mathematical Programming, vol. 50, pp. 367-393, 1991.

[5] A. Dendane, "Maxima and Minima of Functions of Two Variables," 2013.

[6] V. A. Cicirello, "On the Design of an Adaptive Simulated Annealing Algorithm."

[7] J. A. Boyan, "Learning Evaluation Functions for Global Optimization," PhD thesis, 1998.

[8] A. Juels and M. Wattenbergy, "Stochastic Hillclimbing as a Baseline Method for Evaluating Genetic Algorithms," 1984. 